\title{
Search for pair production of first-generation scalar leptoquarks at $\sqrt{s}=13 \mathrm{TeV}$
}

\author{
A. M. Sirunyan et al. \\ (CMS Collaboration)
}

(Received 3 November 2018; published 14 March 2019)

\begin{abstract}
A search for the pair production of first-generation scalar leptoquarks is performed using proton-proton collision data recorded at $13 \mathrm{TeV}$ center-of-mass energy with the CMS detector at the LHC. The data correspond to an integrated luminosity of $35.9 \mathrm{fb}^{-1}$. The leptoquarks are assumed to decay promptly to a quark and either an electron or a neutrino, with branching fractions $\beta$ and $1-\beta$, respectively. The search targets the decay final states comprising two electrons, or one electron and large missing transverse momentum, along with two quarks that are detected as hadronic jets. First-generation scalar leptoquarks with masses below 1435 (1270) $\mathrm{GeV}$ are excluded for $\beta=1.0(0.5)$. These are the most stringent limits on the mass of first-generation scalar leptoquarks to date. The data are also interpreted to set exclusion limits in the context of an $R$-parity violating supersymmetric model, predicting promptly decaying top squarks with a similar dielectron final state.
\end{abstract}

DOI: 10.1103/PhysRevD.99.052002

\section{INTRODUCTION}

The quark and lepton sectors of the standard model (SM) [1-3] are similar: both have the same number of generations composed of electroweak doublets. This could indicate the existence of an additional fundamental symmetry linking the two sectors, as proposed in many scenarios of physics beyond the SM. These include grand unified theories with symmetry groups $\mathrm{SU}(4)$ of the PatiSalam model [4,5], SU(5), SO(10), and SU(15) [6-11]; technicolor [12-14]; superstring-inspired models [15]; and models exhibiting quark and lepton substructures [16]. A common feature of these models is the presence of a new class of bosons, called leptoquarks (LQs), that carry both lepton $(L)$ and baryon numbers $(B)$. In general, LQs have fractional electric charge and are color triplets under $\mathrm{SU}(3)_{\mathrm{C}}$. Their other properties, such as spin, weak isospin, and fermion number $(3 B+L)$, are model dependent.

Direct searches for LQs at colliders are usually interpreted in the context of effective theories that impose constraints on their interactions. In order to ensure renormalizability, these interactions are required to respect SM group symmetries, restricting the couplings of the LQs to SM leptons and quarks only. A detailed account of LQs and their interactions can be found in Ref. [17]. Results from

*Full author list given at the end of the article.

Published by the American Physical Society under the terms of the Creative Commons Attribution 4.0 International license. Further distribution of this work must maintain attribution to the author(s) and the published article's title, journal citation, and DOI. Funded by SCOAP ${ }^{3}$. experiments sensitive to lepton number violation, flavor changing neutral currents, and proton decay allow the existence of three distinct generations of LQs with negligible intergenerational mixing for mass scales accessible at the CERN LHC $[18,19]$. Indirect searches for new physics in rare $B$ meson decays [20-24] by LHCb and Belle suggest a possible breakdown of lepton universality. These anomalies, if confirmed, could provide additional support for LQ-based models [25]. A comprehensive review of LQ phenomenology and experimental constraints on their properties is given in Ref. [26].

We search for the pair production of first-generation scalar LQs that decay promptly. The final state arising from each LQ decay comprises a quark that is detected as a hadronic jet and either an electron or a large missing transverse momentum attributed to the presence of an undetected neutrino. For light-quark final states, the quark flavors cannot be determined from the observed jets. We assume the LQs decay only to $e\left(\nu_{e}\right)$ and up or down quarks. The branching fractions for the LQ decay are expressed in terms of a free parameter $\beta$, where $\beta$ denotes the branching fraction to an electron and a quark, and $1-\beta$ the branching fraction to a neutrino and a quark. For pair production of LQs, we consider two decay modes. The first arises when each LQ decays to an electron and a quark, having an overall branching fraction of $\beta^{2}$. In the second mode one LQ decays to an electron and a quark, and the other to a neutrino and a quark. This mode has a branching fraction of $2 \beta(1-\beta)$. We, therefore, utilize final states with either two high transverse momentum $\left(p_{\mathrm{T}}\right)$ electrons and two high- $p_{\mathrm{T}}$ jets, denoted as $e e \mathrm{jj}$, or one high- $p_{\mathrm{T}}$ electron, large missing transverse momentum, and two high- $p_{\mathrm{T}}$ jets, denoted as $e \nu \mathrm{jj}$. 
Previous experiments at the LEP [27], HERA [28,29], and Tevatron [30,31] colliders have searched for LQ production and placed lower limits of several hundreds of $\mathrm{GeV}$ on allowed LQ masses $\left(m_{\mathrm{LQ}}\right)$ at $95 \%$ confidence level (C.L.). The CMS experiment at the LHC has extended the limits on pair production of first-generation scalar LQs using proton-proton $(p p)$ collision data recorded during 2012 at a center-of-mass energy of $\sqrt{s}=8 \mathrm{TeV}$. Based on a sample corresponding to an integrated luminosity of $19.7 \mathrm{fb}^{-1}$, the lower limit obtained on $m_{\mathrm{LQ}}$ was 1010 (850) $\mathrm{GeV}$ for $\beta=$ 1.0(0.5) [32]. The CMS Collaboration has also published results on a search for singly produced LQs with the final states of either two electrons and one jet, or two muons and one jet [33]. Recently, using a data set of $3.2 \mathrm{fb}^{-1}$ collected at $\sqrt{s}=13 \mathrm{TeV}$, the ATLAS experiment has placed a lower limit on $m_{\mathrm{LQ}}$ of $1100 \mathrm{GeV}$ [34] for $\beta=1.0$.

This analysis is based on data recorded in $p p$ collisions at $\sqrt{s}=13 \mathrm{TeV}$ with the CMS detector, corresponding to an integrated luminosity of $35.9 \mathrm{fb}^{-1}$. At LHC energies, the pair production of LQs would mainly proceed via gluon-gluon fusion with a smaller contribution from quarkantiquark annihilation. The corresponding Feynman diagrams are shown in Fig. 1. The production cross section as a function of $m_{\mathrm{LQ}}$ has been calculated at next-to-leading order (NLO) in perturbation theory [35]. At the LHC, the LQ-lepton-quark Yukawa coupling has negligible effect on the production rate for promptly decaying LQs, which are the focus of our search.

The paper is organized as follows. Section II introduces the CMS detector, and Sec. III describes the data and simulated samples used in the search. The core of the analysis in terms of event reconstruction and selection is discussed in Sec. IV, while the background estimation is presented in Sec. V. Section VI deals with the systematic uncertainties affecting this analysis. Sections VII and VIII describe the results of the LQ search and its interpretation in an exotic scenario of supersymmetry, respectively. We conclude with a summary of the main results in Sec. IX.

\section{THE CMS DETECTOR}

The key feature of the CMS apparatus is a superconducting solenoid of $6 \mathrm{~m}$ diameter, providing a magnetic field of 3.8 T. Within the solenoid volume lie a silicon pixel and microstrip tracker, a lead-tungstate crystal electromagnetic calorimeter (ECAL), and a brass-scintillator hadron calorimeter (HCAL), each composed of a barrel and two end-cap sections. Forward calorimeters extend the pseudorapidity $(\eta)$ coverage provided by the barrel and end-cap detectors. Muons are detected in gas-ionization chambers embedded in the steel flux-return yoke outside the solenoid. The first level of the trigger system [36], composed of custom electronics, uses information from the calorimeters and muon detectors to select the most interesting events in an interval of less than $4 \mu \mathrm{s}$. The high-level trigger processor farm further reduces the event rate from around $100 \mathrm{kHz}$ to $1 \mathrm{kHz}$, before data storage. A detailed description of the CMS detector, along with a definition of the coordinate system used and the relevant kinematic variables, can be found in Ref. [37].

\section{DATA AND SIMULATED SAMPLES}

Events are selected using a combination of triggers requiring either a single electron or a single photon. Electron candidates are required to have a minimum $p_{\mathrm{T}}$ of 27 (115) $\mathrm{GeV}$ for the low (high) threshold trigger. Each of these triggers examines clusters of energy deposited in the ECAL that are matched to tracks reconstructed within a range $|\eta|<2.5$. Cluster shape requirements as well as calorimetric and track-based isolation (only for the low threshold trigger) are also applied. By comparison, the photon trigger requires $p_{\mathrm{T}}>175 \mathrm{GeV}$ without any requirements on track-cluster matching, cluster shape, or
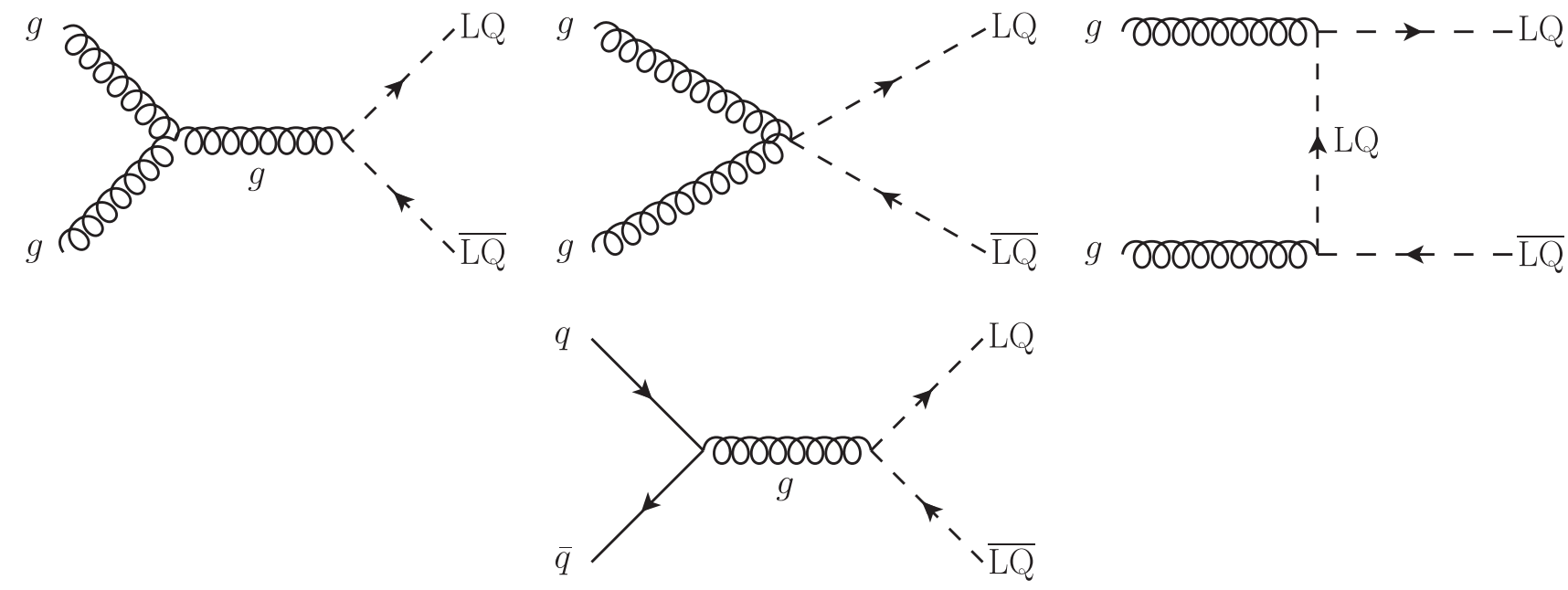

FIG. 1. Leading order Feynman diagrams for the scalar LQ pair production channels at the LHC. 
isolation. The latter three criteria are applied to electron triggers to reduce background rates and are not necessary at high $p_{\mathrm{T}}$. Therefore, the single photon and electron triggers are combined to improve efficiency at high electron $p_{\mathrm{T}}$. Events selected using other single-photon triggers with lower thresholds are used for determining the multijet background.

Monte Carlo (MC) simulation samples of scalar LQ signals are generated using PYTHIA version 8.212 [38] at leading order (LO) with the NNPDF2.3LO parton distribution function (PDF) set [39]. Samples are generated for $m_{\mathrm{LQ}}$ ranging from 200 to $2000 \mathrm{GeV}$ in $50 \mathrm{GeV}$ steps. The LQ is assumed to have quantum numbers corresponding to the combination of an electron $(L=1)$ and an up quark $(B=1 / 3)$, implying it has an electric charge of $-1 / 3$. Possible formation of hadrons containing LQs is not included in the simulation. The cross sections are normalized to the values calculated at NLO [35,40] using the CTEQ6L1 PDF set [41].

The main backgrounds for searches in the eejj and e $\nu \mathrm{jj}$ channels include Drell-Yan $\left(Z / \gamma^{*}\right)$ production with jets, top quark pair production $(t \bar{t})$, single top quark and diboson ( $\mathrm{VV}=W W, W Z$, or $Z Z$ ) production. Additional background contributions arise from $W+$ jets, $\gamma+$ jets, and multijet production, where jets are misidentified as electrons. The $t \bar{t}$ background in the eejj channel as well as the multijet background in both channels are estimated from data, while MC simulated events are used to calculate all other backgrounds. The $Z / \gamma^{*}+$ jets, $W+$ jets, and VV samples are generated at next to leading order (NLO) with MADGRAPH5_aMC@NLO version 2.3.3 using the FxFx merging method [42,43]. Both $t \bar{t}$ and single top quark events are generated at NLO using MADGRAPH5_aMC@NLO, and POWHEG v2 complemented with MADSPIN [44], except for single top quark production in association with a $W$ boson, where events are generated with POWHEG v1 at NLO [45-50], and $s$-channel single top quark production, where MADGRAPH5_aMC@NLO at NLO is used. The $\gamma+$ jets events are generated with MADGRAPH5_aMC@NLO at LO with MLM merging [51]. The NNPDF3.0 at NLO [52] PDF set is used, except for $\gamma+$ jets events that are generated using the LO PDF set.

The $W+$ jets and $Z / \gamma^{*}+$ jets samples are normalized to next-to-NLO (NNLO) inclusive cross sections calculated with FEWZ versions 3.1 and 3.1.b2, respectively [53]. Single top quark samples are normalized to NLO inclusive cross sections [54,55], except for the $t W$ production, where the NNLO calculations of Refs. [56] are used. The calculations from Refs. [57-63] with ToP++2.0 are used to normalize the $t \bar{t}$ sample at NNLO in quantum chromodynamics (QCD) including resummation of the next-tonext-to-leading-logarithmic soft gluon terms.

PYTHIA 8.212 with the CUETP8M1 underlying event tune [64] is used for hadronization and fragmentation in all simulated samples, with the exception of a dedicated tune used for the $t \bar{t}$ sample [65]. All samples include an overlay of minimum bias events (pileup), generated with an approximate distribution for the number of additional $p p$ interactions expected within the same or nearby bunch crossings, and reweighted to match the distribution observed in data. In all cases, the GEANT4 software v.10.00.p02 [66,67] is used to simulate the response of the CMS detector.

\section{EVENT RECONSTRUCTION AND SELECTION}

A particle-flow (PF) algorithm [68] aims to reconstruct and identify each individual particle in a given event, by optimally combining information from the various elements of the CMS detector. The energy of photons is directly obtained from the ECAL measurement. On the other hand, the energy of electrons is determined from a combination of their momentum at the primary interaction vertex as determined by the tracker, the energy of the corresponding ECAL clusters, and the energy sum of all bremsstrahlung photons spatially compatible with originating from the associated track. The momentum of muons is obtained from the curvature of the corresponding track. The energy of charged hadrons is determined from a combination of their momentum measured in the tracker and the matching ECAL and HCAL energy deposits, corrected for zero suppression effects as well as for the response function of the calorimeters to hadronic showers. Finally, the energy of neutral hadrons is obtained from the corresponding corrected ECAL and HCAL energy.

Electrons are identified by spatially matching a reconstructed charged-particle track to a cluster of energy deposits in the ECAL. The ECAL cluster is required to have longitudinal and transverse profiles compatible with those expected from an electromagnetic shower. Electrons used in this analysis are required to have $p_{\mathrm{T}}>50 \mathrm{GeV}$ and $|\eta|<2.5$, excluding the transition regions between barrel and end-cap detectors $1.4442<|\eta|<1.5660$. Additional selection criteria are applied to electron candidates in order to reduce backgrounds while maintaining high efficiency for identification of electrons with large $p_{\mathrm{T}}$ [69]. The absolute difference in $\eta$ between the ECAL cluster seed and the matched track is required to be less than 0.004 (0.006) in the barrel (end cap), and the corresponding quantity in the azimuthal angle, $\phi$, must be less than 0.06 rad. Leptons resulting from the decay of LQs are expected to be isolated from hadronic activity in the event. Requirements are, therefore, applied based on calorimeter energy deposits and tracks in the vicinity of electron candidates. The scalar sum of $p_{\mathrm{T}}$ associated with calorimeter clusters in a cone of radius $\Delta R=\sqrt{(\Delta \eta)^{2}+(\Delta \phi)^{2}}=0.3$ centered on the electron candidate, excluding clusters associated to the candidate itself, must be less than $3 \%$ of the electron $p_{\mathrm{T}}$. A correction to the isolation sum accounts for contributions from pileup interactions. The track-based isolation, calculated as the scalar $p_{\mathrm{T}}$ sum of all tracks in the cone defined 
above, must be less than $5 \mathrm{GeV}$ to reduce misidentification of jets as electrons. At most one layer of the pixel detector may have missing hits along the trajectory of the matched track. The track must also be compatible with originating from the primary $p p$ interaction vertex, which is taken to be the reconstructed vertex with the largest value of summed physics-object $p_{\mathrm{T}}^{2}$. Here the physics objects are the jets, reconstructed using the algorithm [70,71] with the tracks assigned to the vertex as inputs, and the negative vector sum of the $p_{\mathrm{T}}$ of those jets. To correct for the possible difference of electron reconstruction and identification efficiencies between collision and simulated data, appropriate corrections or scale factors are applied to the simulated samples.

Muons are used in defining a control region to estimate the $t \bar{t}$ background contribution. They are identified as tracks in the central tracker consistent with either a track or several hits in the muon system [72]. These muon candidates must have $p_{\mathrm{T}}>35 \mathrm{GeV}$ and $|\eta|<2.4$, and are required to pass a series of identification criteria designed for high- $p_{\mathrm{T}}$ muons as follows. Segments in at least two muon stations must be geometrically matched to a track in the central tracker, with at least one hit from a muon chamber included in the muon track fit. In order to reject muons from decays in flight and increase momentum measurement precision, at least five tracker layers must have hits associated with the muon, and there must be at least one hit in the pixel detector. Isolation is imposed by requiring the $p_{\mathrm{T}}$ sum of tracks in a cone of $\Delta R=0.3$ (excluding the muon itself) divided by the muon $p_{\mathrm{T}}$ to be less than 0.1. For rejection of cosmic ray muons, the transverse impact parameter of the muon track with respect to the primary vertex must be less than $2 \mathrm{~mm}$ and the longitudinal distance of the track formed from tracker system only to the primary vertex must be less than $5 \mathrm{~mm}$. Finally, the relative uncertainty on the $p_{\mathrm{T}}$ measurement from the muon track must be less than $30 \%$.

Jets are reconstructed using the anti- $k_{\mathrm{T}}$ algorithm $[70,71]$ with a distance parameter of 0.4 . Their momentum is determined as the vectorial sum of all particle momenta in the jet, and is found in simulation to be within $5 \%-10 \%$ of the true momentum [73] over the entire $p_{\mathrm{T}}$ spectrum and detector acceptance. Pileup interactions can contribute spurious tracks and calorimeter energy deposits to the jet momentum. To mitigate this effect, tracks identified to be originating from pileup vertices are discarded, while a correction [74] is applied to compensate for the remaining contributions. Jet energy corrections are extracted from simulation to compensate for differences between the true and reconstructed momenta of jets. In situ measurements of the momentum balance in dijet, $\gamma+$ jets, $Z / \gamma^{*}+$ jets, and multijet events are used to estimate and correct for any residual differences in jet energy scale between data and simulation [74]. Additional selection criteria are applied to all jets to remove those potentially affected by spurious energy deposits originating from instrumental effects or reconstruction failures [75]. Jets must have $p_{\mathrm{T}}>50 \mathrm{GeV}$ and $|\eta|<2.4$, and only jets separated from electrons or muons by $\Delta R>0.3$ are retained.

The missing transverse momentum $\left(\vec{p}_{\mathrm{T}}^{\text {miss }}\right)$ is given by the negative vector sum of $p_{\mathrm{T}}$ of all PF candidates in the event. The magnitude of $\vec{p}_{\mathrm{T}}^{\text {miss }}$ is referred to as $p_{\mathrm{T}}^{\text {miss }}$.

To identify $b$ jets arising from top quark decays in the determination of the $e \nu \mathrm{jj}$ background control regions, the combined secondary vertex algorithm is used with the loose working point of Ref. [76]. Based on simulation, the corresponding $b$-jet identification efficiency is above $80 \%$ with a probability of $10 \%$ of misidentifying a lightflavor jet.

\section{A. The $e e \mathrm{jj}$ channel}

For the eejj analysis, we select events with at least two electrons and at least two jets passing the criteria described above. No charge requirements are imposed on the electrons. When additional objects satisfy these requirements, the two highest $p_{\mathrm{T}}$ electrons and jets are considered. Further, there should not be any muon fulfilling the requirements mentioned earlier in this section. The dielectron invariant mass $m_{e e}$ is required to be greater than $50 \mathrm{GeV}$. The $p_{\mathrm{T}}$ of the dielectron system must be greater than $70 \mathrm{GeV}$. The scalar $p_{\mathrm{T}}$ sum over the electrons and two jets, $S_{\mathrm{T}}=p_{\mathrm{T}}\left(e_{1}\right)+p_{\mathrm{T}}\left(e_{2}\right)+p_{\mathrm{T}}\left(\mathrm{j}_{1}\right)+p_{\mathrm{T}}\left(\mathrm{j}_{2}\right)$, must be at least $300 \mathrm{GeV}$. This initial selection is used for the determination of backgrounds in control regions, as explained in Section V.

Final selections are then optimized by maximizing the Punzi criterion for observation of a signal at a significance of five standard deviations [77]. These selections are determined by examining three variables: $m_{e e}, S_{\mathrm{T}}$, and $m_{e j}^{\min }$. The electron-jet pairing is chosen to minimize the difference in the invariant mass of the LQ candidates, and the quantity $m_{e j}^{\min }$ is defined as the smaller of the two masses. Thresholds for the three observables are varied independently, and the Punzi criterion is then calculated at each set of thresholds as well as for each $m_{\mathrm{LQ}}$ hypothesis. The optimized thresholds as a function of $m_{\mathrm{LQ}}$ are shown in Fig. 2 (left). For the $m_{\mathrm{LQ}}$ hypotheses above $1050 \mathrm{GeV}$, the statistical uncertainty in the background prediction becomes large, making an optimization for these masses impossible, and thus the thresholds for the $1050 \mathrm{GeV}$ hypothesis are applied.

\section{B. The $e \nu \mathrm{jj}$ channel}

In the $e \nu \mathrm{jj}$ channel, we select events containing exactly one electron, at least two jets, and $p_{\mathrm{T}}^{\text {miss }}>100 \mathrm{GeV}$. The electron and jets must pass the aforementioned identification criteria. Events with isolated muons are rejected, applying the same criteria as for the eejj channel. The absolute difference in the angle between the $\vec{p}_{\mathrm{T}}^{\text {miss }}$ and the leading $p_{\mathrm{T}}$ jet, $\Delta \phi\left(\vec{p}_{\mathrm{T}}^{\text {miss }}, \mathrm{j}_{1}\right)$, is required to be larger 

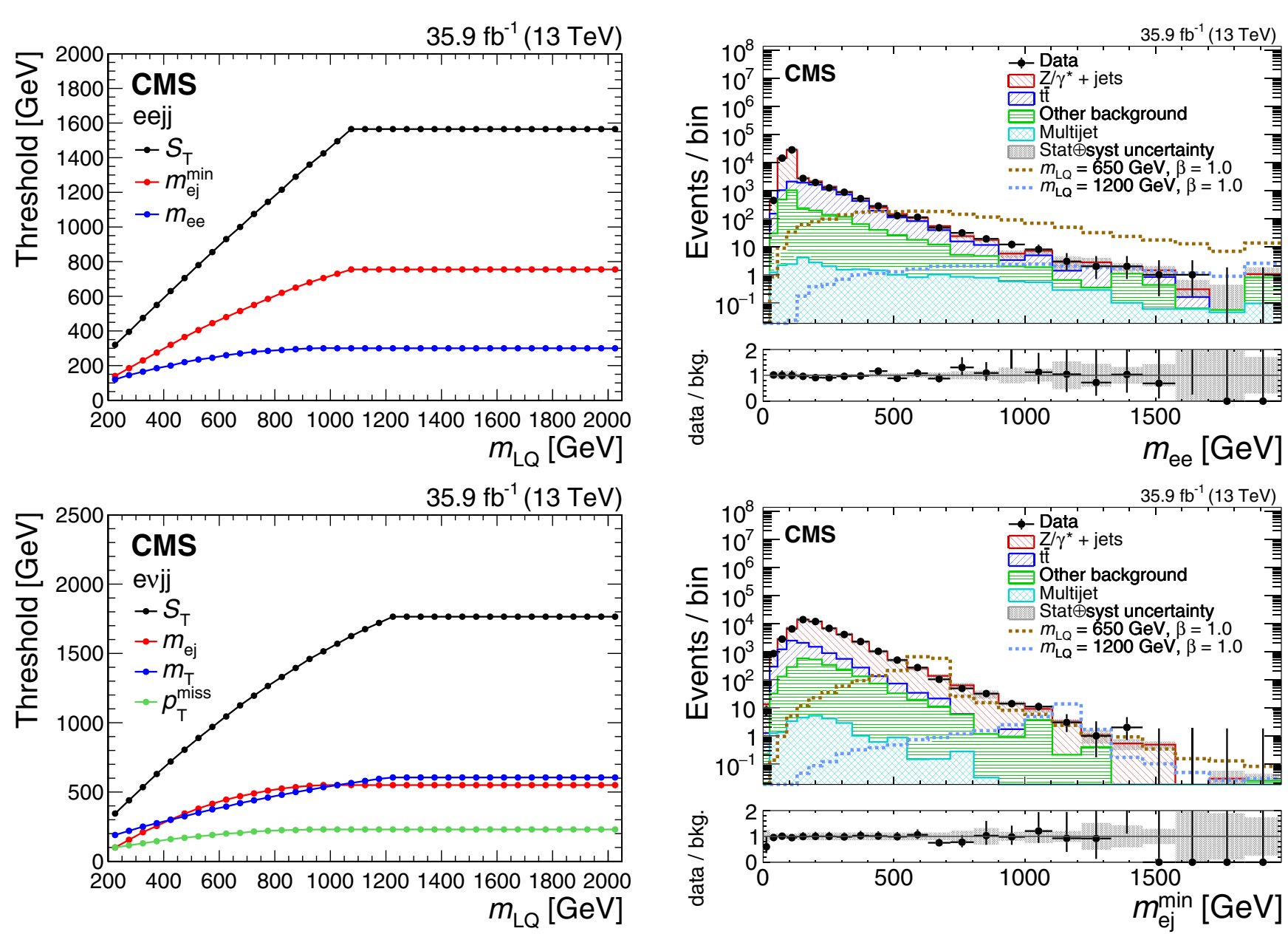

FIG. 2. Optimized threshold values applied for the selection variables in the $e e j \mathrm{j}$ (left) and $e \nu \mathrm{jj}$ (right) channels as a function of $m_{\mathrm{LQ}}$.

than $0.5 \mathrm{rad}$. This helps reject events with $p_{\mathrm{T}}^{\text {miss }}$ arising primarily from instrumental effects. The $\Delta \phi\left(\vec{p}_{\mathrm{T}}^{\text {miss }}, e\right)$ must be greater than $0.8 \mathrm{rad}$ for similar reasons. The $p_{\mathrm{T}}$ and transverse mass of the $\vec{p}_{\mathrm{T}}^{\text {miss }}$-electron system must be greater than 70 and $50 \mathrm{GeV}$, respectively. Here and later, the transverse mass of a two-object system is given by $m_{\mathrm{T}}=\sqrt{2 p_{\mathrm{T}, 1} p_{\mathrm{T}, 2}(1-\cos \Delta \phi)}$, with $\Delta \phi$ being the angle between the $p_{\mathrm{T}}$ vectors of two objects, namely $\vec{p}_{\mathrm{T}}^{\text {miss }}$, electron and jet. The $m_{\mathrm{T}}$ criterion helps suppress the $W+$ jets contribution. Finally, selected events must have $S_{\mathrm{T}}>300 \mathrm{GeV}$, where $S_{\mathrm{T}}=p_{\mathrm{T}}(e)+p_{\mathrm{T}}^{\text {miss }}+p_{\mathrm{T}}\left(\mathrm{j}_{1}\right)+p_{\mathrm{T}}\left(\mathrm{j}_{2}\right)$. This initial selection is used for the determination of backgrounds in control regions, similarly to the eejj channel.

The selection criteria are then optimized in a similar fashion as for the eejj channel, except that four observables are considered for final selections at each $m_{\mathrm{LQ}}$ hypothesis: $S_{\mathrm{T}}, m_{\mathrm{T}}$ of the $\vec{p}_{\mathrm{T}}^{\text {miss }}$-electron system, $p_{\mathrm{T}}^{\text {miss }}$, and the electronjet invariant mass $m_{e \mathrm{j}}$. The $\vec{p}_{\mathrm{T}}^{\text {miss }}$-jet and electron-jet pairing is chosen to minimize the difference in $m_{\mathrm{T}}$ between the two LQ candidates. The optimized thresholds as a function of
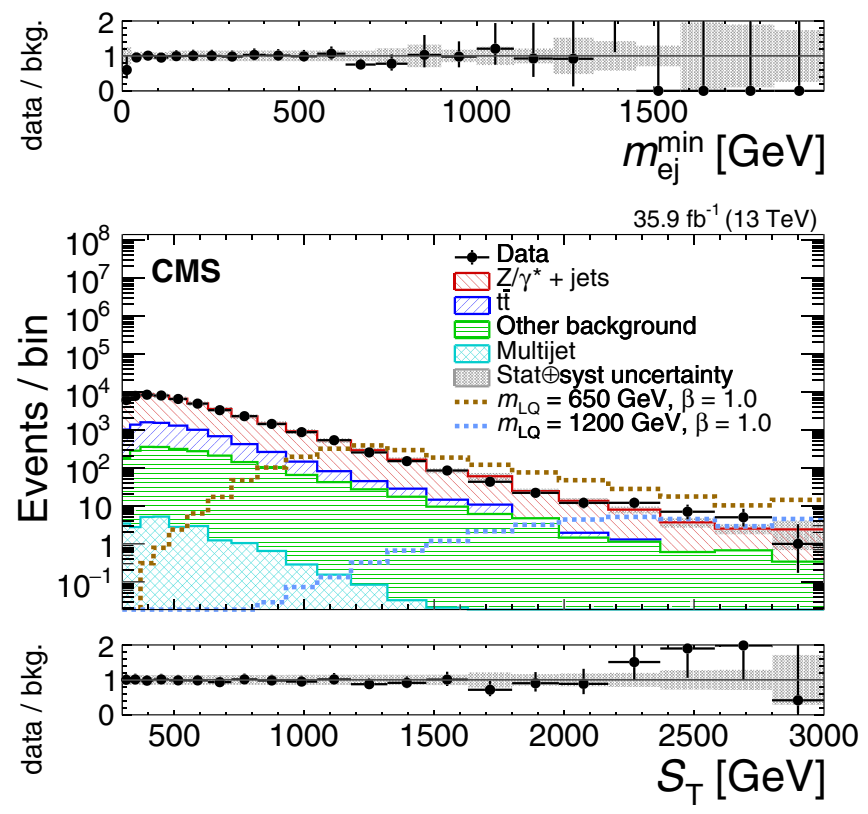

FIG. 3. Data and background comparison for events passing the initial selection requirements for the eejj channel, shown for the variables used for final selection optimization: $m_{e e}$ (upper), $m_{e j}^{\min }$ (lower left), and $S_{\mathrm{T}}$ (lower right). "Other background" includes diboson, single top quark, and $W+$ jets. Signal predictions for $m_{\mathrm{LQ}}=650$ and $1200 \mathrm{GeV}$ hypotheses are overlaid on the plots. The last bin includes all events beyond the upper $x$-axis boundary.

$m_{\mathrm{LQ}}$ are shown in Fig. 2 (right). As with the eejj channel, for the $m_{\mathrm{LQ}}$ hypotheses above $1200 \mathrm{GeV}$, the thresholds for the $1200 \mathrm{GeV}$ hypothesis are used. 


\section{BACKGROUND ESTIMATION}

The SM processes that produce electrons and jets can have final states similar to those of an LQ signal and are, therefore, considered as backgrounds for this search. These include dilepton events from $Z / \gamma^{*}+$ jets, $t \bar{t}$, and $\mathrm{VV}$; single top quark production; and $W+$ jets. Another background arises from multijet production in which at least one jet is misidentified as an electron.

The major backgrounds in the eejj channel are $Z / \gamma^{*}+$ jets and $t \bar{t}$ production. The $Z / \gamma^{*}+$ jets background is estimated from simulation and normalized to the data in a control region that comprises the initial selection plus a window of $80<m_{e e}<100 \mathrm{GeV}$ around the nominal $Z$ boson mass; the latter criterion is applied to enrich the sample with $Z / \gamma^{*}+$ jets events. The $m_{e e}$ distribution is corrected for the presence of non- $Z / \gamma^{*}+$ jets events in the data control region using simulation. The resulting normalization factor applied to the $Z / \gamma^{*}+$ jets simulated events is $R_{Z}=0.97 \pm 0.01$ (stat).

The contribution from $t \bar{t}$ events containing two electrons is estimated using a control region in data, which consists of events containing one electron and one muon, to which all applicable eejj selection criteria are applied. Residual backgrounds from other processes are subtracted using simulated event samples. Corrections for the branching fractions between the two states as well as for the differences in electron/muon identification and isolation efficiencies and acceptances are determined using simulation. The difference in the trigger efficiency between the one- and two-electron final states is corrected by reweighting each event in the $e \mu$ sample with the calculated efficiencies for the single electron final state.

After application of event selection requirements, the background contribution to the eejj channel arising from single top quark production, $W+$ jets, and VV is found to be small and is estimated from simulations.

The multijet background in the eejj channel is estimated using control samples in data. The electron identification requirements for the calorimeter shower profile and trackcluster matching are relaxed to define a loose selection. We measure the probability that an electron candidate that passes the loose selection requirements also satisfies the electron identification and isolation criteria used in the analysis. This probability is obtained as a function of the candidate $p_{\mathrm{T}}$ and $\eta$. The events are required to have
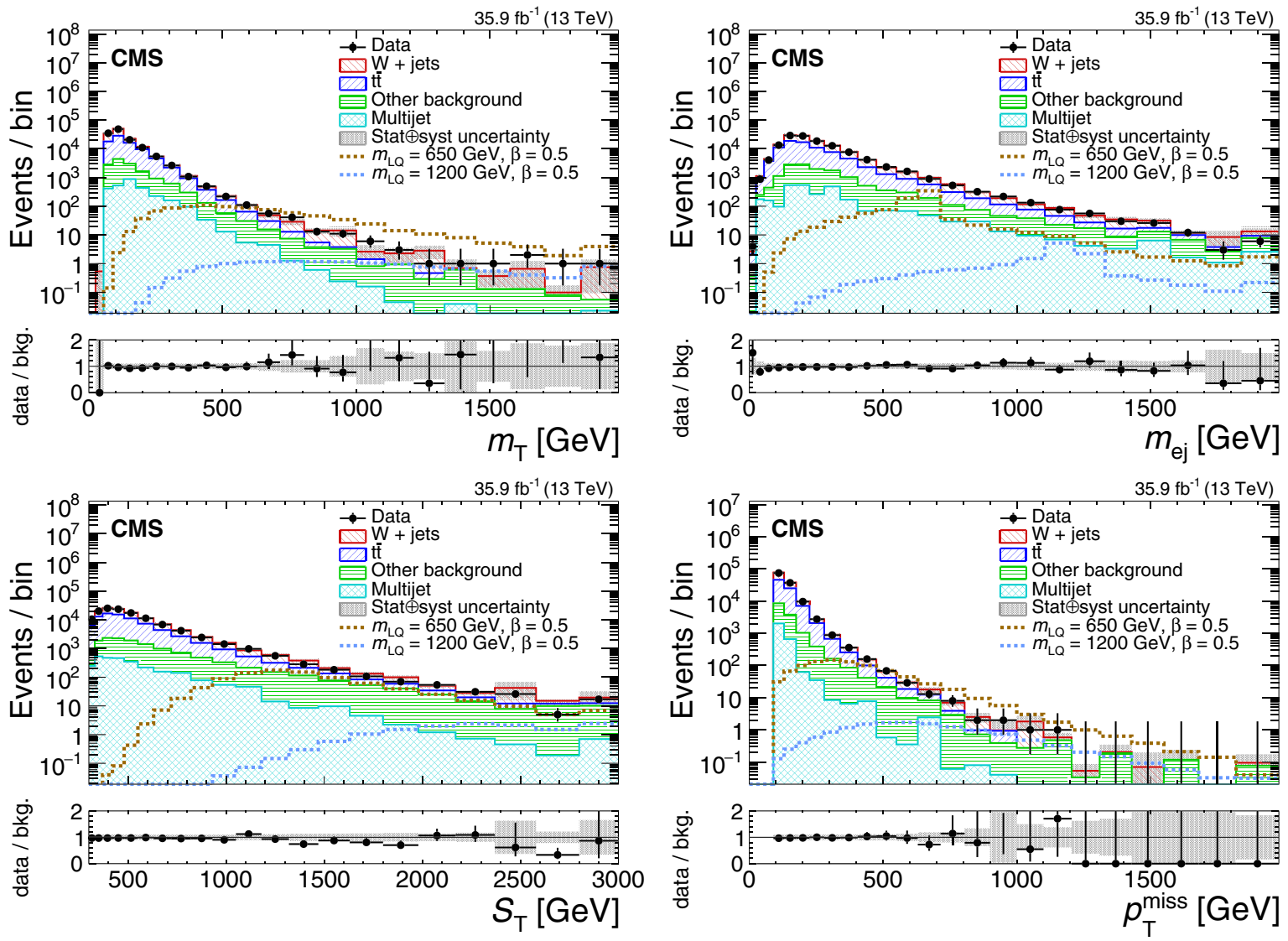

FIG. 4. Data and background for events passing the initial selection requirements in the e $\nu \mathrm{jj}$ channel, shown for the variables used for final selection optimization: $m_{\mathrm{T}}$ (upper left), $m_{e \mathrm{j}}$ (upper right), $S_{\mathrm{T}}$ (lower left), and $p_{\mathrm{T}}^{\text {miss }}$ (lower right). "Other background" includes diboson, single top quark, and $Z / \gamma^{*}+$ jets. Signal predictions for $m_{\mathrm{LQ}}=650$ and $1200 \mathrm{GeV}$ hypotheses are overlaid on the plots. The last bin includes all events beyond the upper $x$-axis boundary. 
exactly one loose electron, at least two jets, and low $p_{\mathrm{T}}^{\text {miss }}$ $(<100 \mathrm{GeV})$. Contributions from electrons satisfying the full identification requirements are removed. The number of such electrons is calculated by comparing the number of candidates that pass the tight selection criteria minus the track-isolation requirement, with those that satisfy the track-isolation requirement but fail one of the other selection criteria. This sample is dominated by QCD multijet events. The distribution of multijet events in the eejj channel following final selections is obtained by applying the measured probability twice to an event sample with two electrons passing loose electron requirements, and two or more jets that satisfy all the requirements of the signal selection. The normalization is obtained by scaling the weighted multijet sample to an orthogonal control region defined by inverting track-isolation requirement for electrons.

Distributions of kinematic variables for the eejj channel in data, including those used in the final selections, have been studied at the initial selection level, and are found to agree with the background models within background estimation uncertainties. The distributions of $S_{\mathrm{T}}, m_{e j}^{\min }$, and $m_{e e}$ are shown in Fig. 3.

The largest background in the e ejj channel comes from $W+$ jets and $t \bar{t}$ production. Single top quark, VV, and
$Z / \gamma^{*}+$ jets backgrounds have small contributions and are estimated from simulations. The QCD multijet background is estimated from control samples in data using the same probability for jets to be misidentified as electrons as is used in the background estimation for the eejj channel. The number of multijet events at the final selection is obtained by selecting events having exactly one loose electron, large $p_{\mathrm{T}}^{\text {miss }}$, and at least two jets satisfying the signal selection criteria, and weighting these with the probability of a jet being misidentified as an electron.

The background contributions from $W+$ jets and $t \bar{t}$ are estimated from simulation and normalized to the data in a control region defined by requiring $50<m_{\mathrm{T}}<110 \mathrm{GeV}$ after the initial selection. Then $b$-tagging information is used to distinguish $W+$ jets from $t \bar{t}$ in the control region. The $W+$ jets contribution is enhanced by requiring zero $b$-tagged jets in the event, while the $t \bar{t}$ control region is defined by requiring at least one $b$-tagged jet in the event. These regions each have a purity of about $75 \%$. The normalization factors for the two backgrounds are determined from these control regions using

$$
\begin{aligned}
& N_{1}=R_{\bar{t} \bar{t}} N_{1, \bar{t}}+R_{W} N_{1, W}+N_{1, \mathrm{O}} \\
& N_{2}=R_{\bar{t} \bar{t}} N_{2, t \bar{t}}+R_{W} N_{2, W}+N_{2, \mathrm{O}},
\end{aligned}
$$

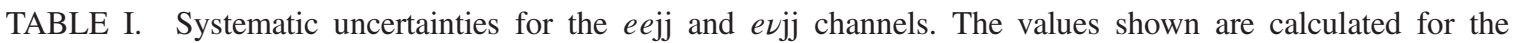
selections used in the $m_{\mathrm{LQ}}=1000 \mathrm{GeV}$ search hypothesis and reflect the variations in the event yields due to each source. Major backgrounds, namely $Z / \gamma^{*}+$ jets $(e e j \mathrm{j}), W+$ jets and $t \bar{t}(e \nu \mathrm{jj})$, are normalized at the initial selection

\begin{tabular}{|c|c|c|c|c|}
\hline \multirow{2}{*}{$\begin{array}{l}\text { Source of } \\
\text { the uncertainty }\end{array}$} & \multicolumn{2}{|c|}{$e e \mathrm{jj}$} & \multicolumn{2}{|c|}{$e \nu \mathrm{jj}$} \\
\hline & Signal (\%) & Background (\%) & Signal $(\%)$ & Background (\%) \\
\hline Electron energy scale & 1.5 & 2.5 & 1.9 & 6.9 \\
\hline Electron energy resolution & 0.2 & 5.3 & 0.1 & 4.9 \\
\hline Electron reconstruction efficiency & 3.0 & 3.0 & 0.6 & 0.8 \\
\hline Electron identification efficiency & 1.3 & 0.3 & 0.6 & 0.1 \\
\hline Trigger & 1.1 & 1.4 & 9.5 & 7.6 \\
\hline Jet energy scale & 0.5 & 0.9 & 0.5 & 2.3 \\
\hline Jet energy resolution & 0.1 & 1.7 & 0.1 & 2.4 \\
\hline$p_{\mathrm{T}}^{\mathrm{miss}}$ & $\cdots$ & $\cdots$ & 0.8 & 13.1 \\
\hline$Z / \gamma^{*}+$ jets shape & $\cdots$ & 5.6 & $\cdots$ & $\cdots$ \\
\hline$Z / \gamma^{*}+$ jets normalization & $\cdots$ & 1.0 & $\cdots$ & $\cdots$ \\
\hline$W+$ jets shape & $\cdots$ & $\cdots$ & $\cdots$ & 7.1 \\
\hline$W+$ jets normalization & $\cdots$ & $\cdots$ & $\cdots$ & 1.1 \\
\hline$W+$ jets sideband selection & $\cdots$ & $\cdots$ & $\cdots$ & 10.0 \\
\hline$W+$ jets $b$ tagging & $\cdots$ & $\cdots$ & $\cdots$ & 3.0 \\
\hline$t \bar{t}$ shape & $\cdots$ & $\cdots$ & $\cdots$ & 10.4 \\
\hline$t \bar{t}$ normalization & $\cdots$ & 1.0 & $\cdots$ & 1.0 \\
\hline$t \bar{t} b$ tagging & $\cdots$ & $\cdots$ & $\cdots$ & 3.0 \\
\hline Diboson shape & $\cdots$ & 3.4 & $\cdots$ & 3.2 \\
\hline QCD multijet & $\cdots$ & $<0.1$ & $\cdots$ & 2.6 \\
\hline Integrated luminosity & 2.5 & 0.6 & 2.5 & 0.5 \\
\hline Pileup & 0.2 & 1.0 & 0.4 & 1.4 \\
\hline PDF & 2.8 & 3.0 & 2.9 & 4.7 \\
\hline
\end{tabular}
level when calculating the effect of shifts for various systematics. 
where $N_{1(2)}$ is the number of events in the $t \bar{t}(W+$ jets) control region in data. The terms $N_{i, t \bar{t}}$ and $N_{i, W}$ are the numbers of $t \bar{t}$ and $W+$ jets events in the simulated samples, while $N_{i, \mathrm{O}}$ is the number of events arising from other background sources, namely diboson, single top quark, $Z / \gamma^{*}+$ jets and multijet. The subscript $i=1,2$ refers to the two control regions described above. The background normalization factors $R_{t \bar{t}}=0.92 \pm 0.01$ (stat) and $R_{W}=$ $0.87 \pm 0.01$ (stat) are then determined by solving Eq. (1).

The observed distributions of kinematic variables for the e $\nu \mathrm{jj}$ channel following the initial selection are found to agree with the background prediction within estimation uncertainties. The distributions of $m_{\mathrm{T}}, m_{e \mathrm{j}}, S_{\mathrm{T}}$, and $p_{\mathrm{T}}^{\text {miss }}$ are shown in Fig. 4.

\section{SYSTEMATIC UNCERTAINTIES}

The sources of systematic uncertainties considered in this analysis are listed in Table I. Uncertainties in the reconstruction of electrons, jets and $p_{\mathrm{T}}^{\text {miss }}$ affect the selected sample of events used in the analysis. The uncertainty due to the electron energy scale is obtained by shifting the electron energy up and down by $2 \%$. The uncertainty in the electron energy resolution is measured by smearing the electron energy by $\pm 10 \%$ [78]. The uncertainties due to electron reconstruction and identification efficiencies are obtained by varying the corresponding scale factors applied to simulated events by \pm 1 standard deviation with respect to their nominal values. The trigger efficiency for electrons is measured by utilizing the tag-and-probe method [79] in data, and parametrized as a function of electron $p_{\mathrm{T}}$ and $\eta$. The corresponding uncertainty depends on the number of data events and is almost entirely statistical in origin for the kinematic range studied in this analysis.

The uncertainty due to the jet energy scale is obtained by varying the nominal scale correction by \pm 1 standard deviation and taking the maximum difference with respect to the nominal event yield. The jet energy resolution models the variation between the reconstructed and generated jets. The corresponding uncertainty is obtained by modifying the parametrization of this difference [74].

To determine uncertainties in $p_{\mathrm{T}}^{\text {miss }}$, we consider up and down shifts in the jet energy scale and resolution, electron energy correction, and the scale corrections applied to the
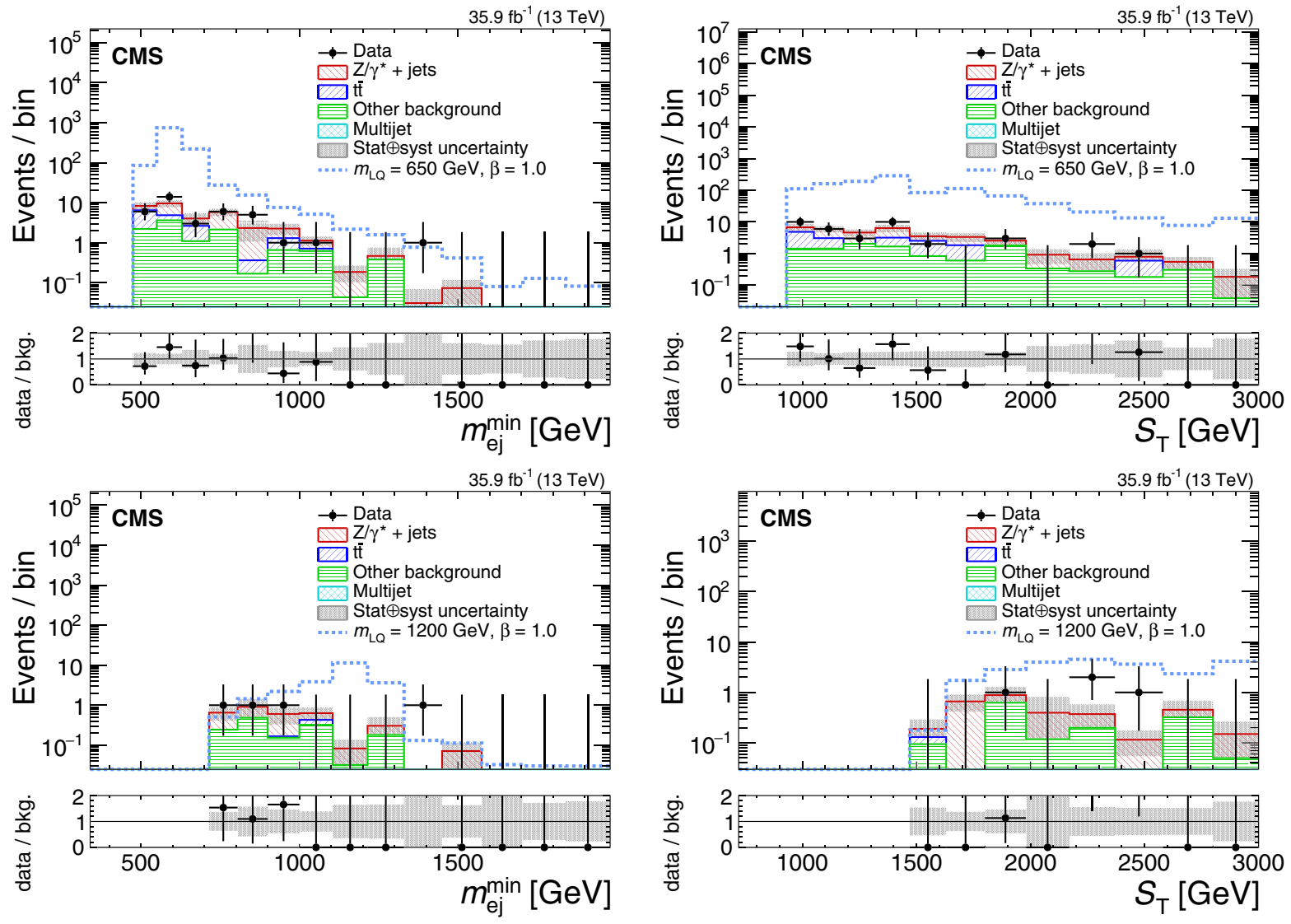

FIG. 5. $m_{e j}^{\min }$ (left) and $S_{\mathrm{T}}$ (right) distributions for events passing the eejj final selection for LQs of mass 650 (upper) and 1200 (lower) GeV. The predicted signal model distributions are shown, along with major backgrounds and "other background" which consists of the sum of the $W+$ jets, diboson, single top quark, and $\gamma+$ jets contributions. The background contributions are stacked, while the signal distributions are plotted unstacked. The dark shaded region indicates the statistical and systematic uncertainty in the total background. The last bin includes all events beyond the upper $x$-axis boundary. 
energy not associated with any PF candidates. For each variation, a new $p_{\mathrm{T}}^{\text {miss }}$ vector is computed for each event. The uncertainties corresponding to different variations in the quantities are then added in quadrature to determine the variation in $p_{\mathrm{T}}^{\text {miss }}$, and the maximum difference of the event yield with respect to nominal is taken as the uncertainty.

Variations in the shape of the $Z / \gamma^{*}+$ jets (eejj channel only), $W+$ jets and $t \bar{t}$ (evjj channel only), and diboson (both channels) backgrounds are determined using simulated samples with renormalization and factorization scales independently varied up and down in the matrix element by a factor of two, yielding eight different combinations. The event yields are then calculated for each of these variations and the maximum variation with respect to nominal is taken as the systematic uncertainty. The corresponding normalization uncertainties are evaluated from the statistical uncertainties in the scale factors obtained while normalizing these backgrounds to data in the control regions. In the $e \nu \mathrm{jj}$ channel, an additional uncertainty of $10 \%$ is included to account for the observed differences associated with the choice of the $m_{\mathrm{T}}$ range, defining the control region used to calculate the normalization scale factors. As described above, $b$-tagging is used to define the control region for $W+$ jets and $t \bar{t}$ normalization in the e $e \mathrm{jj}$ channel; therefore, the uncertainty in the $b$-tagging efficiency (3\%) is taken into account.

The uncertainty in the QCD multijet background is assessed by using an independent data sample. This sample is required to have exactly two electron candidates satisfying loosened criteria applied to the track-cluster matching, the isolation (both track-based and calorimetric), and the shower profile. We compare the number of events in this sample, where one candidate satisfies the electron selection requirements, to that predicted by the multijet background method. This test is repeated on a subsample of the data after applying an $S_{\mathrm{T}}$ threshold of $320 \mathrm{GeV}$, which corresponds to the optimized final selection for an LQ mass of $200 \mathrm{GeV}$. The relative difference of $25 \%$ observed between the results of the two tests is taken as the systematic uncertainty in the probability for a jet to be misidentified as
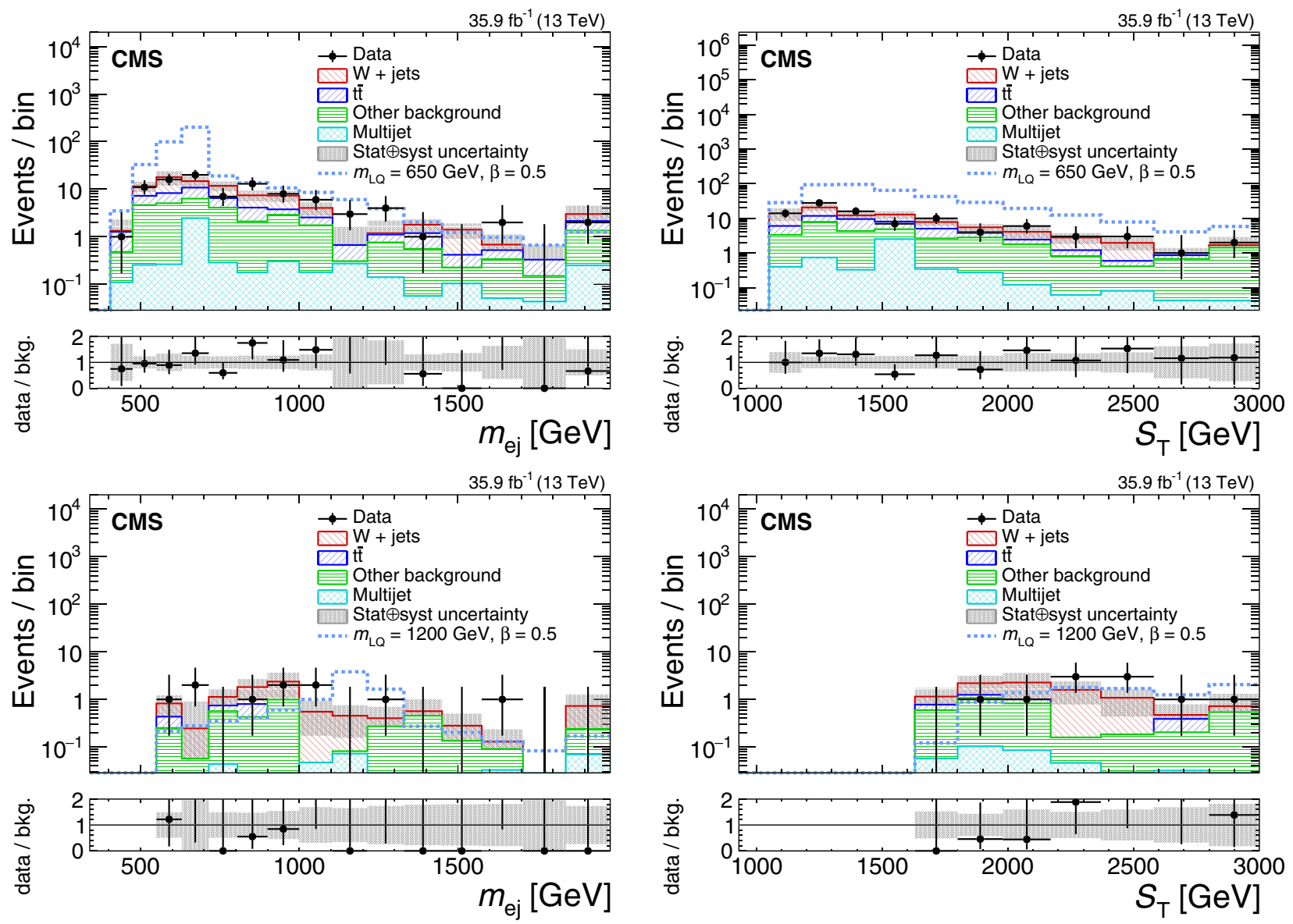

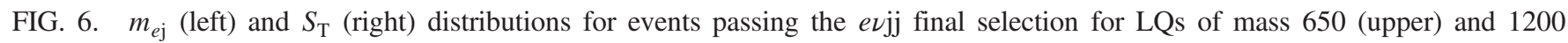
(lower) GeV. The predicted signal model distributions are shown, along with major backgrounds and "other background" which consists of the sum of $Z / \gamma^{*}+$ jets, diboson, single top quark, and $\gamma+$ jets contributions. The background contributions are stacked, while the signal distributions are plotted unstacked. The dark shaded region indicates the statistical and systematic uncertainty in the total background. The last bin includes all events beyond the upper $x$-axis boundary. 
an electron and applied in the e $e \mathrm{jj}$ channel. For the $e e \mathrm{jj}$ case, we assume full correlation between the two electrons and take $50 \%$ as the uncertainty.

The uncertainty in the integrated luminosity is $2.5 \%$ [80]. An uncertainty in the modeling of pileup is evaluated by reweighting the simulated events after varying the inelastic $p p$ cross section by $\pm 4.6 \%$ [81]. The acceptance for both signal and backgrounds, and the expected background cross sections are affected by PDF uncertainties. We estimate this effect by evaluating the complete set of NNPDF 3.0 PDF eigenvectors, following the PDF4LHC prescription $[52,82-85]$.

\section{RESULTS OF THE LEPTOQUARK SEARCH}

After applying the final selection criteria shown in Fig. 2, the data are compared to SM background expectations for both channels and each $m_{\mathrm{LQ}}$ hypothesis. Distributions of $m_{e j}^{\min }$ and $S_{\mathrm{T}}$ are shown in Fig. 5 for the eejj channel with the selections applied for the 650 and $1200 \mathrm{GeV} m_{\mathrm{LQ}}$ hypotheses. Figure 6 shows the corresponding distributions
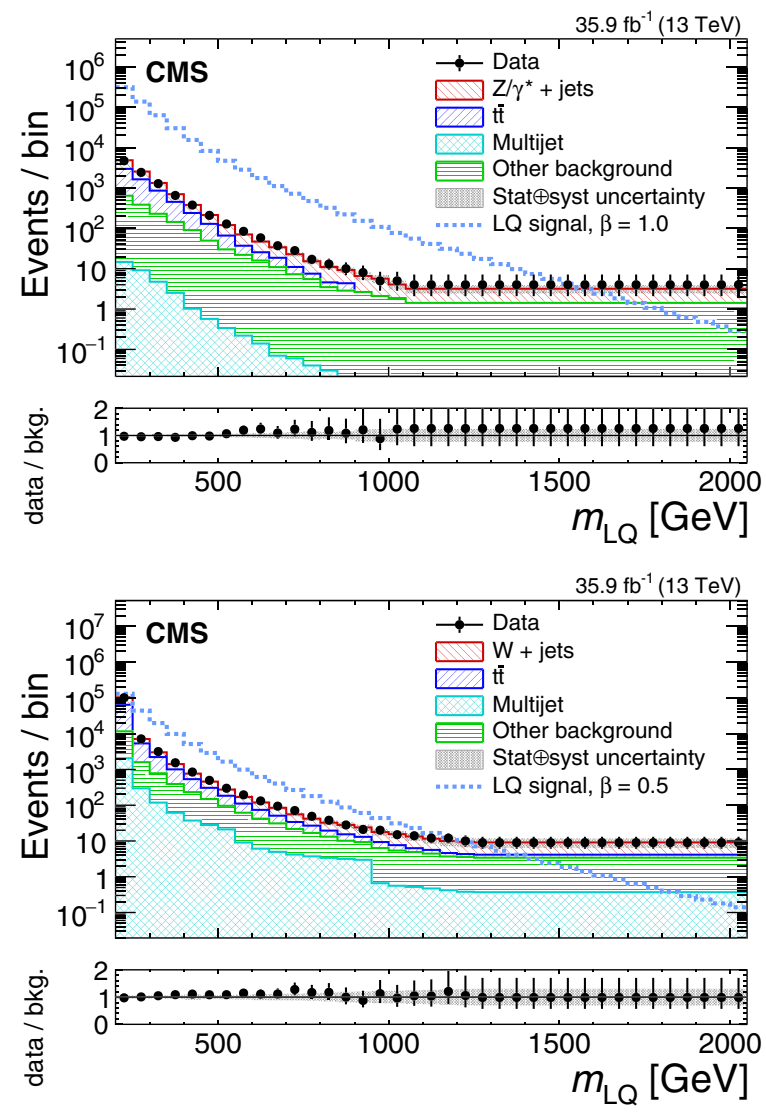

FIG. 7. Data, background, and expected signal yields after applying the final selection criteria for the eejj (left) and e $\nu \mathrm{jj}$ (right) channels. "Other background" includes diboson, single top quark, and $W+$ jets (for the ee jj channel) or $Z / \gamma^{*}+$ jets (for the e $2 \mathrm{jj}$ channel). The bin contents are correlated, because events selected for higher-mass LQ searches are a subset of those selected for lower mass searches. of $m_{e \mathrm{j}}$ and $S_{\mathrm{T}}$ for the $e \nu \mathrm{jj}$ channel for the same mass hypotheses.

Figure 7 shows background, data, and expected signal for each LQ mass point after applying the final selection criteria. Signal efficiency times acceptance, along with tables listing event yields for signal, background, and data are provided in the Appendix. The data are found to be in agreement with SM background expectations in both channels. We set upper limits on the product of the cross section and branching fraction for scalar LQs as a function of $m_{\mathrm{LQ}}$ and $\beta$. The limits are calculated using the asymptotic approximation [86] of the $\mathrm{CL}_{\mathrm{s}}$ modified frequentist approach [87-89]. Systematic uncertainties described in Sec. VI are modeled with log-normal probability density functions, while statistical uncertainties are modeled with gamma functions whose widths are calculated from the number of events in the control regions or simulated samples.

We set upper limits on the production cross section multiplied by the branching fraction $\beta^{2}$ or $2 \beta(1-\beta)$ at 95\% C.L. as a function of $m_{\mathrm{LQ}}$. The expected and observed limits are shown with NLO predictions for the scalar LQ pair production cross section in Fig. 8 for both $e$ ejj and $e \nu \mathrm{jj}$
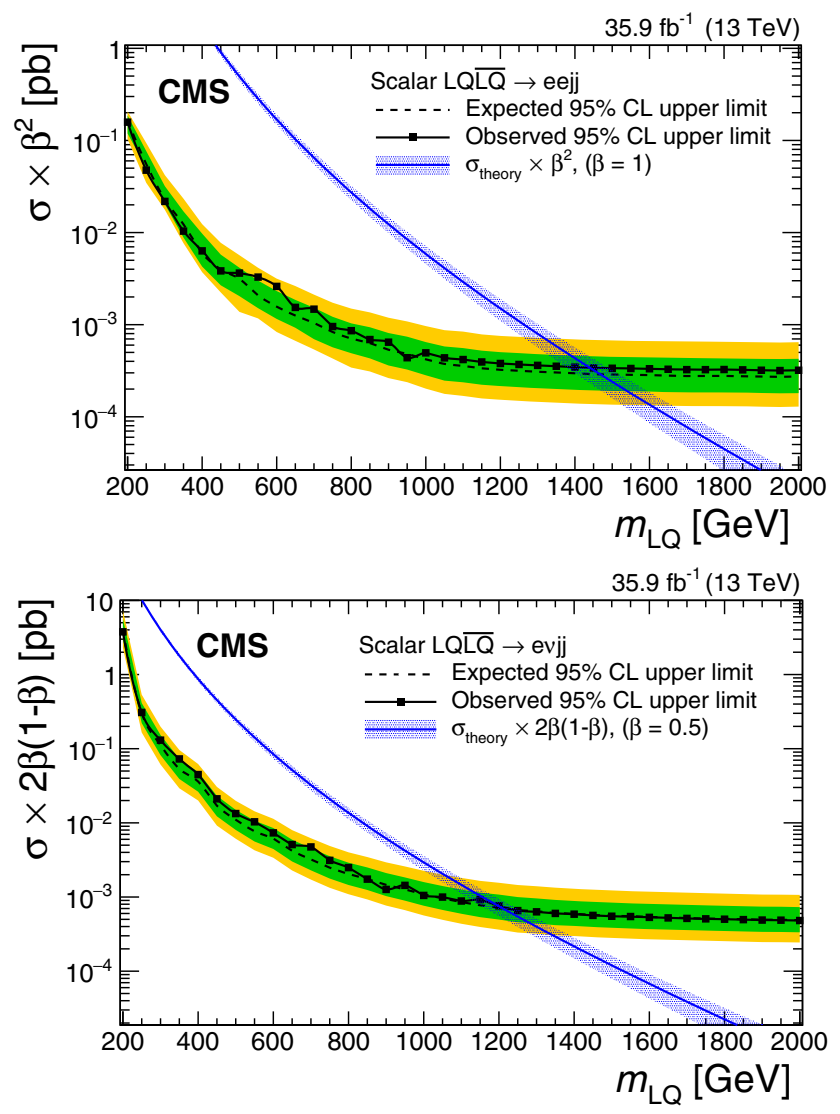

FIG. 8. Observed upper limits for scalar LQ pair-production cross section times $\beta^{2}$ (left) and $\beta(1-\beta)$ (right) at 95\% C.L. obtained with the eejj (left) and $e \nu \mathrm{jj}$ (right) analysis. The median (dashed line), 68\% (inner green band) and 95\% (outer yellow band) confidence-interval expected limits are also shown. 


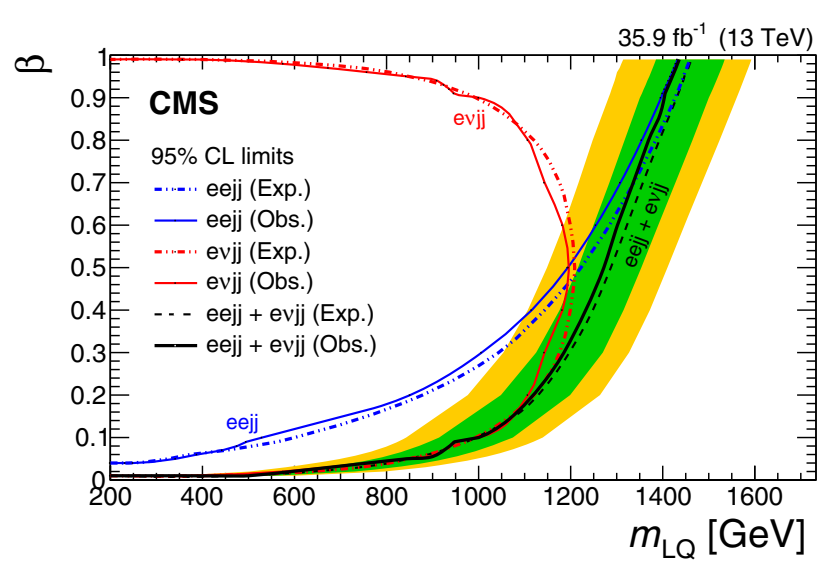

FIG. 9. Expected and observed exclusion limits at 95\% C.L. for pair production of first-generation scalar LQ shown in the $\beta$ versus $m_{\mathrm{LQ}}$ plane for the individual $e e \mathrm{jj}$ and $e \nu \mathrm{jj}$ channels and their combination. The inner green and outer yellow bands represent the $68 \%$ and $95 \%$ confidence intervals on the expected limits.

channels. The observed limits are within two standard deviations of expectations from the background-only hypothesis. The uncertainty in the theoretical prediction for the LQ pair production cross section is calculated as the quadrature sum of the PDF uncertainty in the signal cross section and the uncertainty due to the choice of renormalization and factorization scales. The latter is estimated by independently varying the scales up and down by a factor of two.

Under the assumption $\beta=1.0$, where only the $e e \mathrm{jj}$ channel contributes, first-generation scalar LQs with masses less than $1435 \mathrm{GeV}$ are excluded at 95\% C.L. compared to a median expected limit of $1465 \mathrm{GeV}$. For $\beta=0.5$, using the evjj channel alone, LQ masses are excluded below $1195 \mathrm{GeV}$ with the corresponding expected limit being $1210 \mathrm{GeV}$. As both eejj and evjj decays contribute at $\beta$ values smaller than 1, the LQ mass limit is improved using the combination of the two channels. In this combination, systematic uncertainties are considered to be fully correlated between the channels, while statistical uncertainties are treated as fully uncorrelated. Limits for a range of $\beta$ values from 0 to 1 are set at 95\% C.L. for both $e e \mathrm{jj}$ and $e \nu \mathrm{jj}$ channels, as well as for their combination, as shown in Fig. 9. In the $\beta=0.5$ case, the combination excludes first-generation scalar LQs with masses less than $1270 \mathrm{GeV}$, compared to a median expected value of $1285 \mathrm{GeV}$.

\section{R-PARITY VIOLATING SUPERSYMMETRY INTERPRETATION}

Many new physics models predict the existence of particles with couplings of the type expected for LQs. One such model is $R$-parity violating supersymmetry (RPV SUSY) $[90,91]$, where the superpartners of quarks or 'squarks' can decay into LQ-like final states. For example,

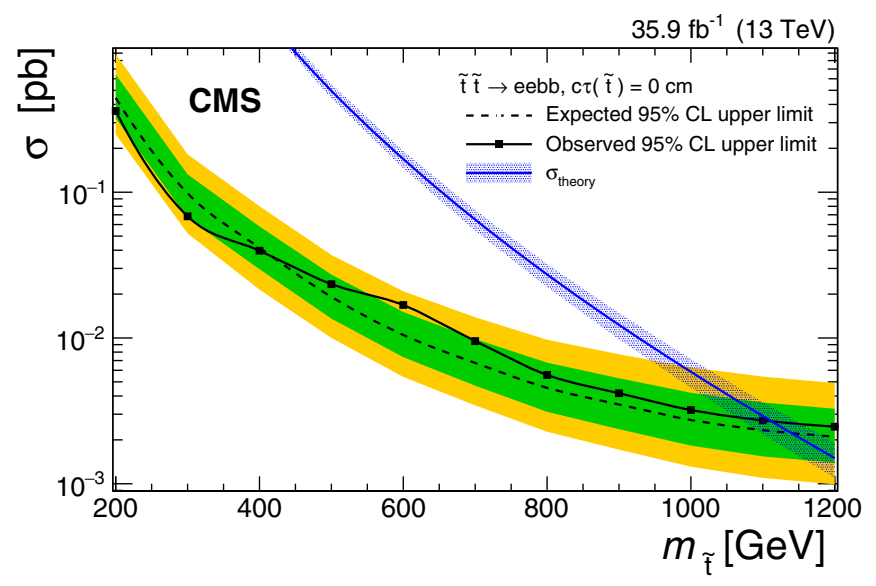

FIG. 10. Expected and observed upper limits at 95\% C.L. on the RPV SUSY $\tilde{t}$ squark pair production cross section as a function of $M_{\tilde{t}}$ for $c \tau=0 \mathrm{~cm}$. The expected limits represent the median values, while the inner green and outer yellow bands are the $68 \%$ and $95 \%$ confidence intervals, respectively.

the top squark $(\tilde{t})$ can decay to a bottom quark and an electron. The topology of the resulting events is similar to an LQ decay and hence these events will pass our nominal selection for the eejj channel.

The analysis is recast in terms of the possible production of prompt top-squark pairs $(c \tau=0 \mathrm{~cm})$, with each $\tilde{t}$ subsequently decaying to a bottom quark and an electron. Limits on the production cross section for $\tilde{t}$ pairs are calculated from the eejj data, accounting for the difference in branching fractions of LQ and $\tilde{t}$ decays to electrons.

Figure 10 shows the expected and observed 95\% C.L. upper limits on the RPV SUSY $\tilde{t}$ pair production cross section as a function of the $\tilde{t}$ squark mass $\left(m_{\tilde{t}}\right)$. The observed exclusion limit is $1100 \mathrm{GeV}$ for $c \tau=0 \mathrm{~cm}$.

\section{SUMMARY}

A search has been performed for the pair production of first-generation scalar leptoquarks in final states consisting of two high-momentum electrons and two jets, or one electron, large missing transverse momentum and two jets. The data sample used in the study corresponds to an integrated luminosity of $35.9 \mathrm{fb}^{-1}$ recorded by the CMS experiment at $\sqrt{s}=13 \mathrm{TeV}$. The data are found to be in agreement with standard model background expectations and a lower limit at $95 \%$ confidence level is set on the scalar leptoquark mass at 1435 (1270) $\mathrm{GeV}$ for $\beta=1.0(0.5)$, where $\beta$ is the branching fraction of the leptoquark decay to an electron and a quark. These results constitute the most stringent limits on the mass of first-generation scalar leptoquarks to date. The data are also interpreted in the context of an $R$-parity violating supersymmetric model with promptly decaying top squarks, which can decay into leptoquark-like final states. Top squarks are excluded for masses below $1100 \mathrm{GeV}$. 


\section{ACKNOWLEDGMENTS}

We congratulate our colleagues in the CERN accelerator departments for the excellent performance of the LHC and thank the technical and administrative staffs at CERN and at other CMS institutes for their contributions to the success of the CMS effort. In addition, we gratefully acknowledge the computing centers and personnel of the Worldwide LHC Computing Grid for delivering so effectively the computing infrastructure essential to our analyses. Finally, we acknowledge the enduring support for the construction and operation of the LHC and the CMS detector provided by the following funding agencies: BMBWF and FWF (Austria); FNRS and FWO (Belgium); CNPq, CAPES, FAPERJ, FAPERGS, and FAPESP (Brazil); MES (Bulgaria); CERN; CAS, MoST, and NSFC (China); COLCIENCIAS (Colombia); MSES and CSF (Croatia); RPF (Cyprus); SENESCYT (Ecuador); MoER, ERC IUT, and ERDF (Estonia); Academy of Finland, MEC, and HIP (Finland); CEA and CNRS/IN2P3 (France); BMBF, DFG, and HGF (Germany); GSRT (Greece); NKFIA (Hungary); DAE and DST (India); IPM (Iran); SFI (Ireland); INFN (Italy); MSIP and NRF (Republic of Korea); MES (Latvia); LAS (Lithuania); MOE and UM (Malaysia); BUAP, CINVESTAV, CONACYT, LNS, SEP, and UASLP-FAI (Mexico); MOS (Montenegro); MBIE (New Zealand); PAEC (Pakistan); MSHE and NSC (Poland); FCT (Portugal); JINR (Dubna); MON, RosAtom, RAS, RFBR, and NRC KI (Russia); MESTD (Serbia); SEIDI, CPAN, PCTI, and FEDER (Spain); MOSTR (Sri Lanka); Swiss Funding Agencies (Switzerland); MST (Taipei); ThEPCenter, IPST, STAR, and NSTDA (Thailand); TUBITAK and TAEK (Turkey); NASU and SFFR (Ukraine); STFC (United Kingdom); DOE and NSF (USA). Individuals have received support from the MarieCurie program and the European Research Council and Horizon 2020 Grant, Contract No. 675440 (European Union); the Leventis Foundation; the A.P. Sloan Foundation; the Alexander von Humboldt Foundation; the Belgian Federal Science Policy Office; the Fonds pour la
Formation à la Recherche dans l'Industrie et dans l'Agriculture (FRIA-Belgium); the Agentschap voor Innovatie door Wetenschap en Technologie (IWTBelgium); the F. R. S.-FNRS and FWO (Belgium) under the "Excellence of Science-EOS"-be.h Project No. 30820817; the Ministry of Education, Youth and Sports (MEYS) of the Czech Republic; the Lendület ("Momentum") Program and the János Bolyai Research Scholarship of the Hungarian Academy of Sciences, the New National Excellence Program ÚNKP, the NKFIA Research Grants No. 123842, No. 123959, No. 124845, No. 124850, and No. 125105 (Hungary); the Council of Science and Industrial Research, India; the HOMING PLUS program of the Foundation for Polish Science, cofinanced from European Union, Regional Development Fund, the Mobility Plus program of the Ministry of Science and Higher Education, the National Science Center (Poland), Contracts Harmonia No. 2014/14/M/ST2/00428, Opus No. 2014/13/ B/ST2/02543, No. 2014/15/B/ST2/03998, and No. 2015/19/ B/ST2/02861, Sonata-bis No. 2012/07/E/ST2/01406; the National Priorities Research Program by Qatar National Research Fund; the Programa Estatal de Fomento de la Investigación Científica y Técnica de Excelencia María de Maeztu, Grant No. MDM-2015-0509 and the Programa Severo Ochoa del Principado de Asturias; the Thalis and Aristeia programs cofinanced by EU-ESF and the Greek NSRF; the Rachadapisek Sompot Fund for Postdoctoral Fellowship, Chulalongkorn University and the Chulalongkorn Academic into Its 2nd Century Project Advancement Project (Thailand); the Welch Foundation, contract C-1845; and the Weston Havens Foundation (USA).

\section{APPENDIX: EFFICIENCIES AND EVENT YIELDS}

In Fig. 11 the product of signal acceptance and efficiency is shown after final optimized selections as a function of

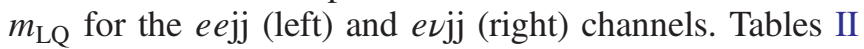
and III list the number of events passing the final selection criteria in data and the various background components as a function of $m_{\mathrm{LQ}}$ for the $e e \mathrm{jj}$ and $e \nu \mathrm{jj}$ channels, respectively.

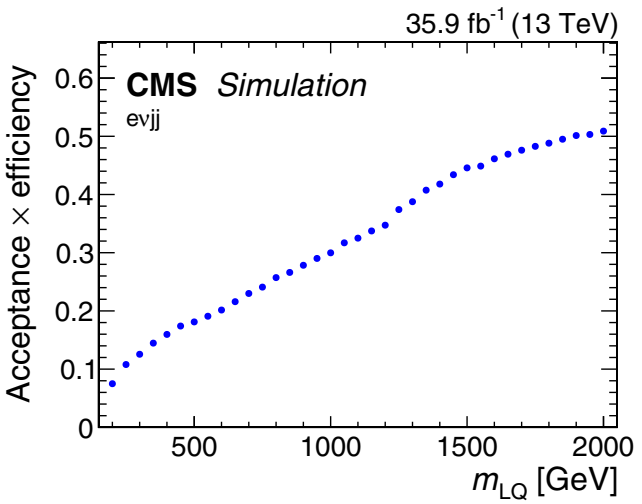

FIG. 11. The product of signal acceptance and efficiency after final optimized selections, as a function of $m_{\mathrm{LQ}}$ for the $e e \mathrm{jj}$ (left) and e $\nu \mathrm{jj}$ (right) channels. 
TABLE II. Event yields after the optimized $e$ jj selections. Uncertainties are statistical except for the total background, where both statistical and systematic uncertainties are shown. An entry of 0.0 quoted for the uncertainty indicates that its value is negligibly small. LQ masses are given in units of $\mathrm{GeV}$ and init. sel. refers to initial selection.

\begin{tabular}{|c|c|c|c|c|c|c|c|}
\hline LQ mass & Signal & $Z / \gamma^{*}+$ jets & $t \bar{t}$ & Multijet & $\mathrm{VV}, W$, single $\mathrm{t}, \gamma+$ jets & Total background & Data \\
\hline init. sel. & $\ldots$ & $41600 \pm 49$ & $7100 \pm 68$ & $26 \pm 0.1$ & $2400 \pm 36$ & $51100 \pm 91 \pm 2700$ & 50585 \\
\hline 200 & $311500 \pm 3300$ & $1900 \pm 16$ & $2300 \pm 39$ & $15 \pm 0.1$ & $630 \pm 18$ & $4800 \pm 46 \pm 120$ & 4709 \\
\hline 250 & $137400 \pm 1200$ & $910 \pm 11$ & $1200 \pm 29$ & $9.1 \pm 0.1$ & $380 \pm 14$ & $2500 \pm 34 \pm 69$ & 2426 \\
\hline 300 & $63160 \pm 510$ & $470 \pm 4.2$ & $630 \pm 22$ & $4.8 \pm 0.0$ & $220_{-9.5}^{+10}$ & $1300_{-24}^{+24} \pm 24$ & 1278 \\
\hline 350 & $30150 \pm 230$ & $250 \pm 2.7$ & $310 \pm 15$ & $2.5 \pm 0.0$ & $140_{-8.6}^{+9.5}$ & $700_{-18}^{+18} \pm 27$ & 652 \\
\hline 400 & $15440 \pm 110$ & $140 \pm 1.8$ & $150 \pm 11$ & $1.0 \pm 0.0$ & $89_{-6.2}^{+7.2}$ & $380_{-13}^{+13} \pm 11$ & 376 \\
\hline 450 & $8260 \pm 60$ & $85 \pm 1.5$ & $79 \pm 7.7$ & $0.6 \pm 0.0$ & $49_{-2.3}^{+2.3}$ & $210_{-8.1}^{+8.2} \pm 5.3$ & 209 \\
\hline 500 & $4700 \pm 33$ & $54 \pm 1.1$ & $36 \pm 5.5$ & $0.3 \pm 0.0$ & $30_{-1.9}^{+2.0}$ & $120_{-5.9}^{+6.0} \pm 4.4$ & 128 \\
\hline 550 & $2830 \pm 19$ & $33 \pm 0.8$ & $15 \pm 4.0$ & $0.2 \pm 0.0$ & $22_{-1.8}^{+1.8}$ & $70_{-4.5}^{+4.5} \pm 2.6$ & 84 \\
\hline 600 & $1750 \pm 12$ & $21 \pm 0.6$ & $9.6 \pm 3.3$ & $0.1 \pm 0.0$ & $16_{-1.6}^{+1.6}$ & $47_{-3.7}^{+3.7} \pm 1.9$ & 58 \\
\hline 650 & $1110 \pm 7.2$ & $15 \pm 0.6$ & $7.7 \pm 2.9$ & $0.1 \pm 0.0$ & $11_{-1.3}^{+1.4}$ & $34_{-3.2}^{+3.2} \pm 1.3$ & 37 \\
\hline 700 & $718 \pm 4.5$ & $12 \pm 0.5$ & $3.7 \pm 2.2$ & $0.1 \pm 0.0$ & $7.3_{-1.2}^{+1.2}$ & $23_{-2.6}^{+2.6} \pm 1.0$ & 28 \\
\hline 750 & $470 \pm 2.9$ & $7.8 \pm 0.3$ & $2.0 \pm 1.9$ & $0.0 \pm 0.0$ & $5.5_{-1.1}^{+1.1}$ & $15_{-2.2}^{+2.2} \pm 0.6$ & 17 \\
\hline 800 & $320 \pm 1.9$ & $6.4 \pm 0.4$ & $1.1_{-0.4}^{+0.5}$ & $0.0 \pm 0.0$ & $3.5_{-0.9}^{+1.1}$ & $11_{-1.1}^{+1.2} \pm 0.6$ & 13 \\
\hline 850 & $220 \pm 1.3$ & $4.9 \pm 0.3$ & $1.5_{-0.5}^{+0.7}$ & $0.0 \pm 0.0$ & $2.8_{-0.6}^{+1.0}$ & $9.2_{-0.8}^{+1.3} \pm 0.5$ & 10 \\
\hline 900 & $150 \pm 0.9$ & $4.0 \pm 0.3$ & $0.0_{-0.0}^{+1.2}$ & $0.0 \pm 0.0$ & $2.6_{-0.5}^{+0.8}$ & $6.6_{-0.6}^{+1.4} \pm 0.4$ & 8 \\
\hline 950 & $110 \pm 0.6$ & $3.6 \pm 0.5$ & $0.0_{-0.0}^{+0.9}$ & $0.0 \pm 0.0$ & $2.1_{-0.5}^{+0.7}$ & $5.7_{-0.7}^{+1.3} \pm 0.3$ & 5 \\
\hline 1000 & $77 \pm 0.4$ & $2.2 \pm 0.1$ & $0.0_{-0.0}^{+0.7}$ & $0.0 \pm 0.0$ & $1.9_{-0.4}^{+0.7}$ & $4.1_{-0.5}^{+1.0} \pm 0.2$ & 5 \\
\hline 1050 & $55 \pm 0.3$ & $1.8 \pm 0.1$ & $0.0_{-0.0}^{+0.3}$ & $0.0 \pm 0.0$ & $1.4_{-0.4}^{+0.6}$ & $3.2_{-0.4}^{+0.7} \pm 0.2$ & 4 \\
\hline 1100 & $41 \pm 0.2$ & $1.8 \pm 0.1$ & $0.0_{-0.0}^{+0.3}$ & $0.0 \pm 0.0$ & $1.4_{-0.4}^{+0.6}$ & $3.2_{-0.4}^{+0.7} \pm 0.2$ & 4 \\
\hline 1150 & $31 \pm 0.2$ & $1.8 \pm 0.1$ & $0.0_{-0.0}^{+0.3}$ & $0.0 \pm 0.0$ & $1.4_{-0.4}^{+0.6}$ & $3.2_{-0.4}^{+0.7} \pm 0.2$ & 4 \\
\hline 1200 & $23 \pm 0.1$ & $1.8 \pm 0.1$ & $0.0_{-0.0}^{+0.3}$ & $0.0 \pm 0.0$ & $1.4_{-0.4}^{+0.6}$ & $3.2_{-0.4}^{+0.7} \pm 0.2$ & 4 \\
\hline 1250 & $17 \pm 0.1$ & $1.8 \pm 0.1$ & $0.0_{-0.0}^{+0.3}$ & $0.0 \pm 0.0$ & $1.4_{-0.4}^{+0.6}$ & $3.2_{-0.4}^{+0.7} \pm 0.2$ & 4 \\
\hline 1300 & $13 \pm 0.1$ & $1.8 \pm 0.1$ & $0.0_{-0.0}^{+0.3}$ & $0.0 \pm 0.0$ & $1.4_{-0.4}^{+0.6}$ & $3.2_{-0.4}^{+0.7} \pm 0.2$ & 4 \\
\hline 1350 & $9.8 \pm 0.0$ & $1.8 \pm 0.1$ & $0.0_{-0.0}^{+0.3}$ & $0.0 \pm 0.0$ & $1.4_{-0.4}^{+0.6}$ & $3.2_{-0.4}^{+0.7} \pm 0.2$ & 4 \\
\hline 1400 & $7.4 \pm 0.0$ & $1.8 \pm 0.1$ & $0.0_{-0.0}^{+0.3}$ & $0.0 \pm 0.0$ & $1.4_{-0.4}^{+0.6}$ & $3.2_{-0.4}^{+0.7} \pm 0.2$ & 4 \\
\hline 1450 & $5.6 \pm 0.0$ & $1.8 \pm 0.1$ & $0.0_{-0.0}^{+0.3}$ & $0.0 \pm 0.0$ & $1.4_{-0.4}^{+0.6}$ & $3.2_{-0.4}^{+0.7} \pm 0.2$ & 4 \\
\hline 1500 & $4.2 \pm 0.0$ & $1.8 \pm 0.1$ & $0.0_{-0.0}^{+0.3}$ & $0.0 \pm 0.0$ & $1.4_{-0.4}^{+0.6}$ & $3.2_{-0.4}^{+0.7} \pm 0.2$ & 4 \\
\hline 1550 & $3.2 \pm 0.0$ & $1.8 \pm 0.1$ & $0.0_{-0.0}^{+0.3}$ & $0.0 \pm 0.0$ & $1.4_{-0.4}^{+0.6}$ & $3.2_{-0.4}^{+0.7} \pm 0.2$ & 4 \\
\hline 1600 & $2.4 \pm 0.0$ & $1.8 \pm 0.1$ & $0.0_{-0.0}^{+0.3}$ & $0.0 \pm 0.0$ & $1.4_{-0.4}^{+0.6}$ & $3.2_{-0.4}^{+0.7} \pm 0.2$ & 4 \\
\hline 1650 & $1.8 \pm 0.0$ & $1.8 \pm 0.1$ & $0.0_{-0.0}^{+0.3}$ & $0.0 \pm 0.0$ & $1.4_{-0.4}^{+0.6}$ & $3.2_{-0.4}^{+0.7} \pm 0.2$ & 4 \\
\hline 1700 & $1.4 \pm 0.0$ & $1.8 \pm 0.1$ & $0.0_{-0.0}^{+0.3}$ & $0.0 \pm 0.0$ & $1.4_{-0.4}^{+0.6}$ & $3.2_{-0.4}^{+0.7} \pm 0.2$ & 4 \\
\hline 1750 & $1.1 \pm 0.0$ & $1.8 \pm 0.1$ & $0.0_{-0.0}^{+0.3}$ & $0.0 \pm 0.0$ & $1.4_{-0.4}^{+0.6}$ & $3.2_{-0.4}^{+0.7} \pm 0.2$ & 4 \\
\hline 1800 & $0.8 \pm 0.0$ & $1.8 \pm 0.1$ & $0.0_{-0.0}^{+0.3}$ & $0.0 \pm 0.0$ & $1.4_{-0.4}^{+0.6}$ & $3.2_{-0.4}^{+0.7} \pm 0.2$ & 4 \\
\hline 1850 & $0.6 \pm 0.0$ & $1.8 \pm 0.1$ & $0.0_{-0.0}^{+0.3}$ & $0.0 \pm 0.0$ & $1.4_{-0.4}^{+0.6}$ & $3.2_{-0.4}^{+0.7} \pm 0.2$ & 4 \\
\hline 1900 & $0.5 \pm 0.0$ & $1.8 \pm 0.1$ & $0.0_{-0.0}^{+0.3}$ & $0.0 \pm 0.0$ & $1.4_{-0.4}^{+0.6}$ & $3.2_{-0.4}^{+0.7} \pm 0.2$ & 4 \\
\hline 1950 & $0.4 \pm 0.0$ & $1.8 \pm 0.1$ & $0.0_{-0.0}^{+0.3}$ & $0.0 \pm 0.0$ & $1.4_{-0.4}^{+0.6}$ & $3.2_{-0.4}^{+0.7} \pm 0.2$ & 4 \\
\hline 2000 & $0.3 \pm 0.0$ & $1.8 \pm 0.1$ & $0.0_{-0.0}^{+0.3}$ & $0.0 \pm 0.0$ & $1.4_{-0.4}^{+0.6}$ & $3.2_{-0.4}^{+0.7} \pm 0.2$ & 4 \\
\hline
\end{tabular}


TABLE III. Event yields after the optimized evjj selections. Uncertainties are statistical except for the total background, where both statistical and systematic uncertainties are shown. An entry of 0.0 quoted for the uncertainty indicates that its value is negligibly small. LQ masses are given in units of $\mathrm{GeV}$ and init. sel. refers to initial selection.

\begin{tabular}{|c|c|c|c|c|c|c|c|}
\hline LQ mass & Signal & $W+$ jets & $t \bar{t}$ & Multijet & $\mathrm{VV}, Z$, single $t, \gamma+$ jets & Total background & Data \\
\hline init. sel. & $\ldots$ & $47900 \pm 160$ & $66900 \pm 110$ & $2800 \pm 15$ & $11300 \pm 72$ & $128900 \pm 210 \pm 8800$ & 125076 \\
\hline 200 & $130800 \pm 1600$ & $40100 \pm 150$ & $52800 \pm 94$ & $2100 \pm 11$ & $9600 \pm 57$ & $104500 \pm 190 \pm 7300$ & 101618 \\
\hline 250 & $44200 \pm 520$ & $1800 \pm 25$ & $3800 \pm 25$ & $300 \pm 2.3$ & $1300 \pm 38$ & $7100 \pm 52 \pm 430$ & 7151 \\
\hline 300 & $19800 \pm 220$ & $800 \pm 15$ & $1400 \pm 16$ & $120 \pm 1.4$ & $660 \pm 37$ & $3000 \pm 43 \pm 170$ & 3164 \\
\hline 350 & $9800 \pm 100$ & $410 \pm 13$ & $610 \pm 10$ & $62 \pm 1.0$ & $330 \pm 11$ & $1400 \pm 20 \pm 88$ & 1539 \\
\hline 400 & $5100 \pm 51$ & $230 \pm 8.9$ & $300 \pm 7.2$ & $37 \pm 0.8$ & $200 \pm 10$ & $760 \pm 15 \pm 74$ & 847 \\
\hline 450 & $2900 \pm 27$ & $150 \pm 6.0$ & $160 \pm 5.2$ & $28 \pm 0.8$ & $120 \pm 9.6$ & $460 \pm 12 \pm 31$ & 496 \\
\hline 500 & $1700 \pm 15$ & $90 \pm 4.1$ & $88 \pm 3.9$ & $21 \pm 0.8$ & $75_{-3.3}^{+3.9}$ & $270_{-6.6}^{+6.9} \pm 21$ & 298 \\
\hline 550 & $990 \pm 8.8$ & $59 \pm 5.2$ & $49 \pm 2.9$ & $9.1 \pm 0.4$ & $53_{-2.9}^{+3.5}$ & $170_{-6.6}^{+6.9} \pm 13$ & 195 \\
\hline 600 & $620 \pm 5.3$ & $45 \pm 5.1$ & $32 \pm 2.3$ & $6.1 \pm 0.4$ & $36_{-2.2}^{+2.8}$ & $120_{-6.0}^{+6.3} \pm 12$ & 132 \\
\hline 650 & $400 \pm 3.3$ & $34 \pm 5.0$ & $20 \pm 1.8$ & $5.0 \pm 0.4$ & $26_{-1.9}^{+2.5}$ & $84_{-5.7}^{+5.9} \pm 8.1$ & 94 \\
\hline 700 & $270 \pm 2.1$ & $22 \pm 1.2$ & $12 \pm 1.5$ & $4.2 \pm 0.5$ & $18_{-1.5}^{+2.1}$ & $56_{-2.5}^{+2.9} \pm 6.1$ & 71 \\
\hline 750 & $180 \pm 1.4$ & $15 \pm 0.9$ & $10 \pm 1.3$ & $3.7 \pm 0.5$ & $13_{-1.3}^{+2.1}$ & $42_{-2.1}^{+2.7} \pm 4.9$ & 49 \\
\hline 800 & $130 \pm 0.9$ & $13 \pm 1.0$ & $6.3 \pm 1.0$ & $3.4 \pm 0.6$ & $9.8_{-1.1}^{+2.0}$ & $32_{-1.9}^{+2.5} \pm 4.6$ & 38 \\
\hline 850 & $86 \pm 0.6$ & $13 \pm 1.1$ & $5.2 \pm 0.9$ & $3.2 \pm 0.7$ & $7.0_{-1.2}^{+2.0}$ & $28_{-2.0}^{+2.6} \pm 4.8$ & 28 \\
\hline 900 & $61 \pm 0.4$ & $11 \pm 1.2$ & $3.8 \pm 0.8$ & $3.0 \pm 0.7$ & $6.3_{-1.1}^{+2.0}$ & $24_{-2.0}^{+2.6} \pm 4.1$ & 21 \\
\hline 950 & $44 \pm 0.3$ & $8.4 \pm 1.0$ & $3.0 \pm 0.7$ & $0.7 \pm 0.1$ & $5.7_{-1.1}^{+2.0}$ & $18_{-1.6}^{+2.3} \pm 3.3$ & 20 \\
\hline 1000 & $31 \pm 0.2$ & $7.9 \pm 0.9$ & $2.2 \pm 0.6$ & $0.6 \pm 0.1$ & $4.8_{-1.1}^{+2.0}$ & $16_{-1.5}^{+2.3} \pm 2.8$ & 15 \\
\hline 1050 & $23 \pm 0.2$ & $7.1 \pm 0.9$ & $1.4_{-0.5}^{+0.7}$ & $0.5 \pm 0.1$ & $4.4_{-1.1}^{+2.0}$ & $13_{-1.4}^{+2.3} \pm 2.5$ & 14 \\
\hline 1100 & $17 \pm 0.1$ & $5.9 \pm 0.8$ & $1.2_{-0.4}^{+0.6}$ & $0.5 \pm 0.1$ & $4.0_{-1.0}^{+2.0}$ & $12_{-1.4}^{+2.3} \pm 2.1$ & 12 \\
\hline 1150 & $12 \pm 0.1$ & $5.4 \pm 0.9$ & $0.9_{-0.4}^{+0.6}$ & $0.4 \pm 0.1$ & $3.3_{-1.0}^{+2.0}$ & $10_{-1.4}^{+2.3} \pm 1.7$ & 12 \\
\hline 1200 & $9.1 \pm 0.1$ & $5.2 \pm 1.1$ & $0.7_{-0.4}^{+0.6}$ & $0.4 \pm 0.1$ & $3.2_{-1.0}^{+2.0}$ & $9.5_{-1.5}^{+2.3} \pm 1.6$ & 10 \\
\hline 1250 & $7.1 \pm 0.0$ & $5.0 \pm 1.1$ & $0.7_{-0.4}^{+0.6}$ & $0.4 \pm 0.1$ & $3.0_{-1.0}^{+2.0}$ & $9.1_{-1.5}^{+2.3} \pm 1.5$ & 9 \\
\hline 1300 & $5.4 \pm 0.0$ & $5.0 \pm 1.1$ & $0.7_{-0.4}^{+0.6}$ & $0.4 \pm 0.1$ & $3.0_{-1.0}^{+2.0}$ & $9.1_{-1.5}^{+2.3} \pm 1.5$ & 9 \\
\hline 1350 & $4.1 \pm 0.0$ & $5.0 \pm 1.1$ & $0.7_{-0.4}^{+0.6}$ & $0.4 \pm 0.1$ & $3.0_{-1.0}^{+2.0}$ & $9.1_{-1.5}^{+2.3} \pm 1.5$ & 9 \\
\hline 1400 & $3.1 \pm 0.0$ & $5.0 \pm 1.1$ & $0.7_{-0.4}^{+0.6}$ & $0.4 \pm 0.1$ & $3.0_{-1.0}^{+2.0}$ & $9.1_{-1.5}^{+2.3} \pm 1.5$ & 9 \\
\hline 1450 & $2.4 \pm 0.0$ & $5.0 \pm 1.1$ & $0.7_{-0.4}^{+0.6}$ & $0.4 \pm 0.1$ & $3.0_{-1.0}^{+2.0}$ & $9.1_{-1.5}^{+2.3} \pm 1.5$ & 9 \\
\hline 1500 & $1.9 \pm 0.0$ & $5.0 \pm 1.1$ & $0.7_{-0.4}^{+0.6}$ & $0.4 \pm 0.1$ & $3.0_{-1.0}^{+2.0}$ & $9.1_{-1.5}^{+2.3} \pm 1.5$ & 9 \\
\hline 1550 & $1.4 \pm 0.0$ & $5.0 \pm 1.1$ & $0.7_{-0.4}^{+0.6}$ & $0.4 \pm 0.1$ & $3.0_{-1.0}^{+2.0}$ & $9.1_{-1.5}^{+2.3} \pm 1.5$ & 9 \\
\hline 1600 & $1.1 \pm 0.0$ & $5.0 \pm 1.1$ & $0.7_{-0.4}^{+0.6}$ & $0.4 \pm 0.1$ & $3.0_{-1.0}^{+2.0}$ & $9.1_{-1.5}^{+2.3} \pm 1.5$ & 9 \\
\hline 1650 & $0.8 \pm 0.0$ & $5.0 \pm 1.1$ & $0.7_{-0.4}^{+0.6}$ & $0.4 \pm 0.1$ & $3.0_{-1.0}^{+2.0}$ & $9.1_{-1.5}^{+2.3} \pm 1.5$ & 9 \\
\hline 1700 & $0.6 \pm 0.0$ & $5.0 \pm 1.1$ & $0.7_{-0.4}^{+0.6}$ & $0.4 \pm 0.1$ & $3.0_{-1.0}^{+2.0}$ & $9.1_{-1.5}^{+2.3} \pm 1.5$ & 9 \\
\hline 1750 & $0.5 \pm 0.0$ & $5.0 \pm 1.1$ & $0.7_{-0.4}^{+0.6}$ & $0.4 \pm 0.1$ & $3.0_{-1.0}^{+2.0}$ & $9.1_{-1.5}^{+2.3} \pm 1.5$ & 9 \\
\hline 1800 & $0.4 \pm 0.0$ & $5.0 \pm 1.1$ & $0.7_{-0.4}^{+0.6}$ & $0.4 \pm 0.1$ & $3.0_{-1.0}^{+2.0}$ & $9.1_{-1.5}^{+2.3} \pm 1.5$ & 9 \\
\hline 1850 & $0.3 \pm 0.0$ & $5.0 \pm 1.1$ & $0.7_{-0.4}^{+0.6}$ & $0.4 \pm 0.1$ & $3.0_{-1.0}^{+2.0}$ & $9.1_{-1.5}^{+2.3} \pm 1.5$ & 9 \\
\hline 1900 & $0.2 \pm 0.0$ & $5.0 \pm 1.1$ & $0.7_{-0.4}^{+0.6}$ & $0.4 \pm 0.1$ & $3.0_{-1.0}^{+2.0}$ & $9.1_{-1.5}^{+2.3} \pm 1.5$ & 9 \\
\hline 1950 & $0.2 \pm 0.0$ & $5.0 \pm 1.1$ & $0.7_{-0.4}^{+0.6}$ & $0.4 \pm 0.1$ & $3.0_{-1.0}^{+2.0}$ & $9.1_{-1.5}^{+2.3} \pm 1.5$ & 9 \\
\hline 2000 & $0.1 \pm 0.0$ & $5.0 \pm 1.1$ & $0.7_{-0.4}^{+0.6}$ & $0.4 \pm 0.1$ & $3.0_{-1.0}^{+2.0}$ & $9.1_{-1.5}^{+2.3} \pm 1.5$ & 9 \\
\hline
\end{tabular}


[1] S. L. Glashow, Partial-symmetries of weak interactions, Nucl. Phys. 22, 579 (1961).

[2] S. Weinberg, A Model of Leptons, Phys. Rev. Lett. 19, 1264 (1967).

[3] A. Salam, Weak and electromagnetic interactions, in Elementary Particle Physics: Relativistic Groups and Analyticity, edited by N. Svartholm (Almqvist \& Wiksell, Stockholm, 1968), p. 367, proceedings of the eighth Nobel symposium.

[4] J. C. Pati and A. Salam, Unified lepton-hadron symmetry and a gauge theory of the basic interactions, Phys. Rev. D 8, 1240 (1973).

[5] J. C. Pati and A. Salam, Lepton number as the fourth "color", Phys. Rev. D 10, 275 (1974).

[6] H. Georgi and S. Glashow, Unity of all Elementary-Particle Forces, Phys. Rev. Lett. 32, 438 (1974).

[7] H. Murayama and T. Yanagida, A viable SU(5) GUT with light leptoquark bosons, Mod. Phys. Lett. A 07, 147 (1992).

[8] H. Fritzsch and P. Minkowski, Unified interactions of leptons and hadrons, Ann. Phys. (N.Y.) 93, 193 (1975).

[9] G. Senjanović and A. Sokorac, Light lepto-quarks in SO (10), Z. Phys. C 20, 255 (1983).

[10] P. H. Frampton and B.-H. Lee, SU(15) Grand Unification, Phys. Rev. Lett. 64, 619 (1990).

[11] P. H. Frampton and T. W. Kephart, Higgs sector and proton decay in SU(15) grand unification, Phys. Rev. D 42, 3892 (1990).

[12] S. Dimopoulos and L. Susskind, Mass without scalars, Nucl. Phys. B155, 237 (1979).

[13] S. Dimopoulos, Technicoloured signatures, Nucl. Phys. B168, 69 (1980).

[14] E. Eichten and K. Lane, Dynamical breaking of weak interaction symmetries, Phys. Lett. 90B, 125 (1980).

[15] J. L. Hewett and T. G. Rizzo, Low-energy phenomenology of superstring-inspired $\mathrm{E}_{6}$ models, Phys. Rep. 183, 193 (1989).

[16] B. Schrempp and F. Schrempp, Light leptoquarks, Phys. Lett. 153B, 101 (1985).

[17] W. Buchmüller, R. Rückl, and D. Wyler, Leptoquarks in lepton-quark collisions, Phys. Lett. B 191, 442 (1987).

[18] W. Buchmüller and D. Wyler, Constraints on SU(5)-type leptoquarks, Phys. Lett. B 177, 377 (1986).

[19] O. Shanker, $\pi \ell 2, \mathrm{~K} \ell 3$, and $\mathrm{K}^{0}-\overline{\mathrm{K}}^{0}$ constraints on leptoquarks and supersymmetric particles, Nucl. Phys. B204, 375 (1982).

[20] LHCb Collaboration, Measurement of Form-Factor-Independent Observables in the Decay $B^{0} \rightarrow K^{* 0} \mu^{+} \mu^{-}$, Phys. Rev. Lett. 111, 191801 (2013).

[21] LHCb Collaboration, Test of Lepton Universality Using $B^{+} \rightarrow K^{+} \ell^{+} \ell^{-}$Decays, Phys. Rev. Lett. 113, 151601 (2014).

[22] LHCb Collaboration, Angular analysis of the $B^{0} \rightarrow$ $K^{* 0} \mu^{+} \mu^{-}$decay using $3 \mathrm{fb}^{-1}$ of integrated luminosity, J. High Energy Phys. 02 (2016) 104.

[23] LHCb Collaboration, Test of lepton universality with $B^{0} \rightarrow$ $K^{* 0} \ell^{+} \ell^{-}$decays, J. High Energy Phys. 08 (2017) 055.

[24] S. Wehle et al. (Belle Collaboration), Lepton-flavordependent angular analysis of $B \rightarrow K^{*} \ell^{+} \ell^{-}$, Phys. Rev. Lett. 118, 111801 (2017).
[25] G. Hiller, Lepton nonuniversality anomalies and implications, arXiv:1804.02011.

[26] I. Doršner, S. Fajfer, A. Greljo, J. F. Kamenik, and N. Košnik, Physics of leptoquarks in precision experiments and at particle colliders, Phys. Rep. 641, 1 (2016).

[27] G. Abbiendi et al. (OPAL Collaboration), Search for pair-produced leptoquarks in $e^{+} e^{-}$interactions at $\sqrt{s} \simeq 189-209 \mathrm{GeV}$, Eur. Phys. J. C 31, 281 (2003).

[28] S. Chekanov et al. (ZEUS Collaboration), Search for resonance decays to lepton + jet at DESY HERA and limits on leptoquarks, Phys. Rev. D 68, 052004 (2003).

[29] F. D. Aaron et al. (H1 Collaboration), Search for first generation leptoquarks in ep collisions at HERA, Phys. Lett. B 704, 388 (2011).

[30] D. Acosta et al. (CDF Collaboration), Search for firstgeneration scalar leptoquarks in $p \bar{p}$ collisions at $\sqrt{s}=1.96$ TeV, Phys. Rev. D 72, 051107 (2005).

[31] V. M. Abazov et al. (D0 Collaboration), Search for pair production of first-generation leptoquarks in $p \bar{p}$ collisions at $\sqrt{s}=1.96 \mathrm{TeV}$, Phys. Lett. B 681, 224 (2009).

[32] CMS Collaboration, Search for pair production of first and second generation leptoquarks in proton-proton collisions at $\sqrt{s}=8 \mathrm{TeV}$, Phys. Rev. D 93, 032004 (2016).

[33] CMS Collaboration, Search for single production of scalar leptoquarks in proton-proton collisions at $\sqrt{s}=8 \mathrm{TeV}$, Phys. Rev. D 93, 032005 (2016); Phys. Rev. DErratum 95, 039906(E) (2017).

[34] ATLAS Collaboration, Search for scalar leptoquarks in pp collisions at $\sqrt{s}=13 \mathrm{TeV}$ with the ATLAS experiment, New J. Phys. 18, 093016 (2016).

[35] M. Krämer, T. Plehn, M. Spira, and P. M. Zerwas, Pair production of scalar leptoquarks at the CERN LHC, Phys. Rev. D 71, 057503 (2005).

[36] CMS Collaboration, The CMS trigger system, J. Instrum. 12, P01020 (2017).

[37] CMS Collaboration, The CMS experiment at the CERN LHC, J. Instrum. 3, S08004 (2008).

[38] T. Sjöstrand, S. Ask, J. R. Christiansen, R. Corke, N. Desai, P. Ilten, S. Mrenna, S. Prestel, C. O. Rasmussen, and P.Z. Skands, An introduction to PYTHIA 8.2, Comput. Phys. Commun. 191, 159 (2015).

[39] R. D. Ball, V. Bertone, S. Carrazza, C. S. Deans, L. Del Debbio, S. Forte, A. Guffanti, N. P. Hartland, J. I. Latorre, J. Rojo, and M. Ubiali, Parton distributions with LHC data, Nucl. Phys. B867, 244 (2013).

[40] M. Krämer, T. Plehn, M. Spira, and P. M. Zerwas, Pair Production of Scalar Leptoquarks at the Fermilab Tevatron, Phys. Rev. Lett. 79, 341 (1997).

[41] J. Pumplin, D. R. Stump, J. Huston, H. L. Lai, P. Nadolsky, and W. K. Tung, New generation of parton distributions with uncertainties from global QCD analysis, J. High Energy Phys. 07 (2002) 012.

[42] J. Alwall, R. Frederix, S. Frixione, V. Hirschi, F. Maltoni, O. Mattelaer, H. S. Shao, T. Stelzer, P. Torrielli, and M. Zaro, The automated computation of tree-level and next-toleading order differential cross sections, and their matching to parton shower simulations, J. High Energy Phys. 07 (2014) 079.

[43] R. Frederix and S. Frixione, Merging meets matching in MC@NLO, J. High Energy Phys. 12 (2012) 061. 
[44] P. Artoisenet, R. Frederix, O. Mattelaer, and R. Rietkerk, Automatic spin-entangled decays of heavy resonances in Monte Carlo simulations, J. High Energy Phys. 03 (2013) 015.

[45] P. Nason, A new method for combining NLO QCD with shower Monte Carlo algorithms, J. High Energy Phys. 11 (2004) 040.

[46] S. Frixione, P. Nason, and C. Oleari, Matching NLO QCD computations with parton shower simulations: The POWHEG method, J. High Energy Phys. 11 (2007) 070.

[47] S. Alioli, P. Nason, C. Oleari, and E. Re, A general framework for implementing NLO calculations in shower Monte Carlo programs: The POWHEG BOX, J. High Energy Phys. 06 (2010) 043.

[48] S. Alioli, P. Nason, C. Oleari, and E. Re, NLO single-top production matched with shower in POWHEG: $s$ - and $t$-channel contributions, J. High Energy Phys. 09 (2009) 111; J. High Energy Phys.Erratum 02 (2010) 11(E).

[49] E. Re, Single-top Wt-channel production matched with parton showers using the POWHEG method, Eur. Phys. J. C 71, 1547 (2011).

[50] S. Frixione, P. Nason, and G. Ridolfi, A positive-weight next-to-leading-order Monte Carlo for heavy flavour hadroproduction, J. High Energy Phys. 09 (2007) 126.

[51] J. Alwall, S. Höche, F. Krauss, N. Lavesson, L. Lönnblad, F. Maltoni, M. L. Mangano, M. Moretti, C. G. Papadopoulos, F. Piccinini, S. Schumann, M. Treccani, J. Winter, and M. Worek, Comparative study of various algorithms for the merging of parton showers and matrix elements in hadronic collisions, Eur. Phys. J. C 53, 473 (2008).

[52] R. D. Ball et al. (NNPDF Collaboration), Parton distributions for the LHC Run II, J. High Energy Phys. 04 (2015) 040.

[53] Y. Li and F. Petriello, Combining QCD and electroweak corrections to dilepton production in the framework of the FEWZ simulation code, Phys. Rev. D 86, 094034 (2012).

[54] M. Aliev, H. Lacker, U. Langenfeld, S. Moch, P. Uwer, and M. Wiedermann, HATHOR-hadronic top and heavy quarks cross section calculator, Comput. Phys. Commun. 182, 1034 (2011).

[55] P. Kant, O. M. Kind, T. Kintscher, T. Lohse, T. Martini, S. Mölbitz, P. Rieck, and P. Uwer, HATHOR for single topquark production: Updated predictions and uncertainty estimates for single top-quark production in hadronic collisions, Comput. Phys. Commun. 191, 74 (2015).

[56] N. Kidonakis, Top quark production, in Helmholtz International Summer School on Physics of Heavy Quarks and Hadrons (HQ 2013): JINR, Dubna, Russia, 2013, http://www-library.desy.de/preparch/desy/proc/proc13-03/ Kidonakis.pdf.

[57] M. Beneke, P. Falgari, S. Klein, and C. Schwinn, Hadronic top-quark pair production with NNLL threshold resummation, Nucl. Phys. B855, 695 (2012).

[58] M. Cacciari, M. Czakon, M. Mangano, A. Mitov, and P. Nason, Top-pair production at hadron colliders with next-tonext-to-leading logarithmic soft-gluon resummation, Phys. Lett. B 710, 612 (2012).

[59] P. Bärnreuther, M. Czakon, and A. Mitov, Percent-LevelPrecision Physics at the Tevatron: Next-to-Next-to-Leading
Order QCD Corrections to $q \bar{q} \rightarrow t \bar{t}+\mathrm{x}$, Phys. Rev. Lett. 109, 132001 (2012).

[60] M. Czakon and A. Mitov, NNLO corrections to top-pair production at hadron colliders: The all-fermionic scattering channels, J. High Energy Phys. 12 (2012) 054.

[61] M. Czakon and A. Mitov, NNLO corrections to top pair production at hadron colliders: The quark-gluon reaction, J. High Energy Phys. 01 (2013) 080.

[62] M. Czakon, P. Fiedler, and A. Mitov, Total Top-Quark PairProduction Cross Section at Hadron Colliders Through $\mathcal{O}\left(\alpha_{S}^{4}\right)$, Phys. Rev. Lett. 110, 252004 (2013).

[63] M. Czakon and A. Mitov, Top++: A program for the calculation of the top-pair cross-section at hadron colliders, Comput. Phys. Commun. 185, 2930 (2014).

[64] CMS Collaboration, Event generator tunes obtained from underlying event and multiparton scattering measurements, Eur. Phys. J. C 76, 155 (2016).

[65] CMS Collaboration, Investigations of the impact of the parton shower tuning in PYTHIA 8 in the modelling of $t \bar{t}$ at $\sqrt{s}=8$ and $13 \mathrm{TeV}$, CMS Physics Analysis Summary Report No. CMS-PAS-TOP-16-021, 2016, https://cds.cern .ch/record/2235192.

[66] S. Agostinelli et al. (GEANT4 Collaboration), GEANT4-a simulation toolkit, Nucl. Instrum. Methods Phys. Res., Sect. A 506, 250 (2003).

[67] J. Allison et al., Geant4 developments and applications, IEEE Trans. Nucl. Sci. 53, 270 (2006).

[68] CMS Collaboration, Particle-flow reconstruction and global event description with the CMS detector, J. Instrum. 12, P10003 (2017).

[69] CMS Collaboration, Performance of electron reconstruction and selection with the CMS detector in proton-proton collisions at $\sqrt{s}=8 \mathrm{TeV}$, J. Instrum. 10, P06005 (2015).

[70] M. Cacciari, G. P. Salam, and G. Soyez, The anti- $k_{\mathrm{T}}$ jet clustering algorithm, J. High Energy Phys. 04 (2008) 063.

[71] M. Cacciari, G. P. Salam, and G. Soyez, FastJet user manual, Eur. Phys. J. C 72, 1896 (2012).

[72] CMS Collaboration, Performance of the CMS muon detector and muon reconstruction with proton-proton collisions at $\sqrt{s}=13 \mathrm{TeV}$, J. Instrum. 13, P06015 (2018).

[73] CMS Collaboration, Jet algorithms performance in $13 \mathrm{TeV}$ data, CMS Physics Analysis Summary Report No. CMSPAS-JME-16-003, 2017, http://cds.cern.ch/record/2256875.

[74] CMS Collaboration, Jet energy scale and resolution in the CMS experiment in pp collisions at $8 \mathrm{TeV}$, J. Instrum. 12, P02014 (2017).

[75] CMS Collaboration, Performance of missing transverse momentum reconstruction algorithms in proton-proton collisions at $\sqrt{s}=8 \mathrm{TeV}$ with the CMS detector, CMS Physics Analysis Summary Report No. CMS-PAS-JME-12-002, 2013, http://cds.cern.ch/record/1543527.

[76] CMS Collaboration, Identification of heavy-flavour jets with the CMS detector in pp collisions at $13 \mathrm{TeV}$, J. Instrum. 13, P05011 (2018).

[77] G. Punzi, Sensitivity of searches for new signals and its optimization, in PHYSTAT 2003: Statistical problems in particle physics, astrophysics and cosmology (Stanford, USA, 2003), Conference C03-09-08, arXiv:physics/ 0308063 . 
[78] CMS Collaboration, Performance of photon reconstruction and identification with the CMS detector in proton-proton collisions at $\sqrt{s}=8 \mathrm{TeV}$, J. Instrum. 10, P08010 (2015).

[79] CMS Collaboration, Measurements of inclusive $\mathrm{W}$ and $\mathrm{Z}$ cross sections in pp collisions at $\sqrt{s}=7 \mathrm{TeV}$, J. High Energy Phys. 01 (2011) 080.

[80] CMS Collaboration, CMS luminosity measurements for the 2016 data taking period, CMS physics analysis summary Report No. CMS-PAS-LUM-17-001, 2017, http://cds.cern .ch/record/2257069.

[81] CMS Collaboration, Measurement of the inelastic protonproton cross section at $\sqrt{s}=13 \mathrm{TeV}$, J. High Energy Phys. 07 (2018) 161.

[82] M. Botje, J. Butterworth, A. Cooper-Sarkar, A. de Roeck, J. Feltesse, S. Forte, A. Glazov, J. Huston, R. McNulty, T. Sjöstrand, and R.S. Thorne, The PDF4LHC Working Group Interim Recommendations, arXiv:1101.0538.

[83] S. Alekhin et al., The PDF4LHC Working Group Interim Report, arXiv:1101.0536.

[84] M. R. Whalley, D. Bourilkov, and R. C. Group, The Les Houches accord PDFs (LHAPDF) and LHAGLUE, in HERA and the LHC: A Workshop on the implications of HERA for LHC physics. Proceedings, Part B, arXiv:hep-ph/ 0508110 .

[85] D Bourilkov, R. C. Group, and M. R. Whalley, LHAPDF: PDF use from the Tevatron to the LHC, in TeV4LHC
Workshop-4th meeting Batavia, Illinois, 2005, arXiv: hep-ph/0605240.

[86] G. Cowan, K. Cranmer, E. Gross, and O. Vitells, Asymptotic formulae for likelihood-based tests of new physics, Eur. Phys. J. C 71, 1554 (2011); Eur. Phys. J. CErratum 73, 2501(E) (2013).

[87] T. Junk, Confidence level computation for combining searches with small statistics, Nucl. Instrum. Methods Phys. Res., Sect. A 434, 435 (1999).

[88] A. L. Read, Presentation of search results: The $\mathrm{CL}_{\mathrm{s}}$ technique, in Advanced statistical techniques in particle physics proceedings, conference, Durham, UK, 2002, DOI: 10.1088/0954-3899/28/10/313.

[89] ATLAS and CMS Collaboration, The LHC Higgs Combination Group, Procedure for the LHC Higgs boson search combination in Summer 2011, Technical Reports No. CMS NOTE-2011/005 and No. ATL-PHYS-PUB-2011-11, 2011, https://cds.cern.ch/record/1379837.

[90] H. K. Dreiner, An introduction to explicit $R$-parity violation, Pramana 51, 123 (1998).

[91] R. Barbier, C. Bérat, M. Besanćon, M. Chemtob, A. Deandrea, E. Dudas, P. Fayet, S. Lavignac, G. Moreau, E. Perez, and Y. Sirois, $R$-parity-violating supersymmetry, Phys. Rep. 420, 1 (2005).

A. M. Sirunyan, ${ }^{1}$ A. Tumasyan, ${ }^{1}$ W. Adam, ${ }^{2}$ F. Ambrogi, ${ }^{2}$ E. Asilar, ${ }^{2}$ T. Bergauer, ${ }^{2}$ J. Brandstetter, ${ }^{2}$ M. Dragicevic, ${ }^{2}$ J. Erö, ${ }^{2}$ A. Escalante Del Valle, ${ }^{2}$ M. Flechl, ${ }^{2}$ R. Frühwirth, ${ }^{2, a}$ V. M. Ghete, ${ }^{2}$ J. Hrubec, ${ }^{2}$ M. Jeitler, ${ }^{2, a}$ N. Krammer, ${ }^{2}$ I. Krätschmer, ${ }^{2}$ D. Liko, ${ }^{2}$ T. Madlener, ${ }^{2}$ I. Mikulec, ${ }^{2}$ N. Rad, ${ }^{2}$ H. Rohringer, ${ }^{2}$ J. Schieck, ${ }^{2, a}$ R. Schöfbeck, ${ }^{2}$ M. Spanring, ${ }^{2}$ D. Spitzbart, ${ }^{2}$ A. Taurok, ${ }^{2}$ W. Waltenberger, ${ }^{2}$ J. Wittmann, ${ }^{2}$ C.-E. Wulz, ${ }^{2, a}$ M. Zarucki, ${ }^{2}$ V. Chekhovsky, ${ }^{3}$ V. Mossolov, ${ }^{3}$ J. Suarez Gonzalez, ${ }^{3}$ E. A. De Wolf, ${ }^{4}$ D. Di Croce, ${ }^{4}$ X. Janssen, ${ }^{4}$ J. Lauwers, ${ }^{4}$ M. Pieters, ${ }^{4}$ H. Van Haevermaet, ${ }^{4}$ P. Van Mechelen, ${ }^{4}$ N. Van Remortel, ${ }^{4}$ S. Abu Zeid, ${ }^{5}$ F. Blekman, ${ }^{5}$ J. D'Hondt, ${ }^{5}$ J. De Clercq,${ }^{5}$ K. Deroover, ${ }^{5}$ G. Flouris, ${ }^{5}$ D. Lontkovskyi, ${ }^{5}$ S. Lowette, ${ }^{5}$ I. Marchesini, ${ }^{5}$ S. Moortgat,${ }^{5}$ L. Moreels, ${ }^{5}$ Q. Python, ${ }^{5}$ K. Skovpen, ${ }^{5}$ S. Tavernier, ${ }^{5}$ W. Van Doninck, ${ }^{5}$ P. Van Mulders, ${ }^{5}$ I. Van Parijs, ${ }^{5}$ D. Beghin, ${ }^{6}$ B. Bilin, ${ }^{6}$ H. Brun, ${ }^{6}$ B. Clerbaux,${ }^{6}$ G. De Lentdecker, ${ }^{6}$ H. Delannoy, ${ }^{6}$ B. Dorney, ${ }_{6}^{6}$ G. Fasanella, ${ }^{6}$ L. Favart, ${ }^{6}$ R. Goldouzian, ${ }^{6}$ A. Grebenyuk, ${ }^{6}$ A. K. Kalsi, ${ }^{6}$ T. Lenzi, ${ }^{6}$ J. Luetic, ${ }^{6}$ N. Postiau, ${ }^{6}$ E. Starling, ${ }^{6}$ L. Thomas, ${ }^{6}$ C. Vander Velde, ${ }^{6}$ P. Vanlaer, ${ }^{6}$ D. Vannerom, ${ }^{6}$ Q. Wang, ${ }^{6}$ T. Cornelis, ${ }^{7}$ D. Dobur, ${ }^{7}$ A. Fagot, ${ }^{7}$ M. Gul, ${ }^{7}$ I. Khvastunov, ${ }^{7, b}$ D. Poyraz, ${ }^{7}$ C. Roskas, ${ }^{7}$ D. Trocino, ${ }^{7}$ M. Tytgat, ${ }^{7}$ W. Verbeke, ${ }^{7}$ B. Vermassen, ${ }^{7}$ M. Vit, ${ }^{7}$ N. Zaganidis, ${ }^{7}$ H. Bakhshiansohi, ${ }^{8}$ O. Bondu, ${ }^{8}$ S. Brochet, ${ }^{8}$ G. Bruno,${ }^{8}$ C. Caputo, ${ }^{8}$ P. David, ${ }^{8}$ C. Delaere, ${ }^{8}$ M. Delcourt, ${ }^{8}$ A. Giammanco, ${ }^{8}$ G. Krintiras, ${ }^{8}$ V. Lemaitre, ${ }^{8}$ A. Magitteri, ${ }^{8}$ K. Piotrzkowski, ${ }^{8}$ A. Saggio, ${ }^{8}$ M. Vidal Marono, ${ }^{8}$ S. Wertz ${ }^{8}$ J. Zobec, ${ }^{8}$ F. L. Alves, ${ }^{9}$ G. A. Alves, ${ }^{9}$ M. Correa Martins Junior, ${ }^{9}$ G. Correia Silva, ${ }^{9}$ C. Hensel, ${ }^{9}$ A. Moraes, ${ }^{9}$ M. E. Pol,,${ }^{9}$ P. Rebello Teles, ${ }^{9}$ E. Belchior Batista Das Chagas, ${ }^{10}$ W. Carvalho, ${ }^{10}$ J. Chinellato, ${ }^{10, \mathrm{c}}$ E. Coelho, ${ }^{10}$ E. M. Da Costa, ${ }^{10}$ G. G. Da Silveira, ${ }^{10, d}$ D. De Jesus Damiao, ${ }^{10}$ C. De Oliveira Martins, ${ }^{10}$ S. Fonseca De Souza, ${ }^{10}$ H. Malbouisson, ${ }^{10}$ D. Matos Figueiredo, ${ }^{10}$ M. Melo De Almeida, ${ }^{10}$ C. Mora Herrera, ${ }^{10}$ L. Mundim, ${ }^{10}$ H. Nogima, ${ }^{10}$ W. L. Prado Da Silva, ${ }^{10}$ L. J. Sanchez Rosas, ${ }^{10}$ A. Santoro, ${ }^{10}$ A. Sznajder, ${ }^{10}$ M. Thiel, ${ }^{10}$ E. J. Tonelli Manganote,,${ }^{10, c}$ F. Torres Da Silva De Araujo, ${ }^{10}$ A. Vilela Pereira, ${ }^{10}$ S. Ahuja, ${ }^{11 a}$ C. A. Bernardes,${ }^{11 a}$ L. Calligaris,${ }^{11 a}$

T. R. Fernandez Perez Tomei, ${ }^{11 \mathrm{a}}$ E. M. Gregores, ${ }^{11 \mathrm{a}, 11 \mathrm{~b}}$ P. G. Mercadante, ${ }^{1 \mathrm{a}, 11 \mathrm{~b}}$ S. F. Novaes, ${ }^{11 \mathrm{a}}$ Sandra S. Padula, ${ }^{11 \mathrm{a}}$ A. Aleksandrov, ${ }^{12}$ R. Hadjiiska, ${ }^{12}$ P. Iaydjiev, ${ }^{12}$ A. Marinov, ${ }^{12}$ M. Misheva, ${ }^{12}$ M. Rodozov, ${ }^{12}$ M. Shopova, ${ }^{12}$ G. Sultanov, ${ }^{12}$ A. Dimitrov, ${ }^{13}$ L. Litov,${ }^{13}$ B. Pavlov, ${ }^{13}$ P. Petkov, ${ }^{13}$ W. Fang, ${ }^{14, \mathrm{e}}$ X. Gao ${ }^{14, \mathrm{e}}$ L. Yuan, ${ }^{14}$ M. Ahmad, ${ }^{15}$ J. G. Bian, ${ }^{15}$ G. M. Chen, ${ }^{15}$ H. S. Chen, ${ }^{15}$ M. Chen, ${ }^{15}$ Y. Chen, ${ }^{15}$ C. H. Jiang, ${ }^{15}$ D. Leggat,${ }^{15}$ H. Liao, ${ }^{15}$ Z. Liu, ${ }^{15}$ F. Romeo, ${ }^{15}$ S. M. Shaheen, ${ }^{15, \mathrm{f}}$ A. Spiezia, ${ }^{15}$ J. Tao, ${ }^{15}$ Z. Wang, ${ }^{15}$ E. Yazgan, ${ }^{15}$ H. Zhang, ${ }^{15}$ S. Zhang, ${ }^{15, f}$ J. Zhao, ${ }^{15}$ Y. Ban, ${ }^{16}$ G. Chen, ${ }^{16}$ 
A. Levin, ${ }^{16}$ J. Li,${ }^{16}$ L. Li, ${ }^{16}$ Q. Li,${ }^{16}$ Y. Mao, ${ }^{16}$ S. J. Qian, ${ }^{16}$ D. Wang, ${ }^{16}$ Y. Wang, ${ }^{17}$ C. Avila, ${ }^{18}$ A. Cabrera, ${ }^{18}$ C. A. Carrillo Montoya, ${ }^{18}$ L. F. Chaparro Sierra, ${ }^{18}$ C. Florez, ${ }^{18}$ C. F. González Hernández, ${ }^{18}$ M. A. Segura Delgado, ${ }^{18}$ B. Courbon, ${ }^{19}$ N. Godinovic, ${ }^{19}$ D. Lelas, ${ }^{19}$ I. Puljak, ${ }^{19}$ T. Sculac, ${ }^{19}$ Z. Antunovic, ${ }^{20}$ M. Kovac, ${ }^{20}$ V. Brigljevic,${ }^{21}$ D. Ferencek, ${ }^{21}$ K. Kadija, ${ }^{21}$ B. Mesic, ${ }^{21}$ A. Starodumov, ${ }^{21, g}$ T. Susa, ${ }^{21}$ M. W. Ather, ${ }^{22}$ A. Attikis, ${ }^{22}$ M. Kolosova, ${ }^{22}$ G. Mavromanolakis, ${ }^{22}$ J. Mousa, ${ }^{22}$ C. Nicolaou, ${ }^{22}$ F. Ptochos, ${ }^{22}$ P. A. Razis, ${ }^{22}$ H. Rykaczewski, ${ }^{22}$ M. Finger, ${ }^{23, h}$ M. Finger Jr., ${ }^{23, h}$ E. Ayala, ${ }^{24}$ E. Carrera Jarrin, ${ }^{25}$ Y. Assran, ${ }^{26, i, j}$ S. Elgammal, ${ }^{26, i}$ S. Khalil, ${ }^{26, k}$ S. Bhowmik, ${ }^{27}$ A. Carvalho Antunes De Oliveira, ${ }^{27}$ R. K. Dewanjee, ${ }^{27}$ K. Ehataht, ${ }^{27}$ M. Kadastik, ${ }^{27}$ M. Raidal, ${ }^{27}$ C. Veelken, ${ }^{27}$ P. Eerola, ${ }^{28}$ H. Kirschenmann, ${ }^{28}$ J. Pekkanen, ${ }^{28}$ M. Voutilainen, ${ }^{28}$ J. Havukainen, ${ }^{29}$ J. K. Heikkilä, ${ }^{29}$ T. Järvinen, ${ }^{29}$ V. Karimäki, ${ }^{29}$ R. Kinnunen, ${ }^{29}$ T. Lampén, ${ }^{29}$ K. Lassila-Perini, ${ }^{29}$ S. Laurila, ${ }^{29}$ S. Lehti, ${ }^{29}$ T. Lindén, ${ }^{29}$ P. Luukka, ${ }^{29}$ T. Mäenpää, ${ }^{29}$ H. Siikonen, ${ }^{29}$ E. Tuominen, ${ }^{29}$ J. Tuominiemi, ${ }^{29}$ T. Tuuva, ${ }^{30}$ M. Besancon, ${ }^{31}$ F. Couderc,${ }^{31}$ M. Dejardin, ${ }^{31}$ D. Denegri, ${ }^{31}$ J. L. Faure, ${ }^{31}$ F. Ferri, ${ }^{31}$ S. Ganjour, ${ }^{31}$ A. Givernaud,${ }^{31}$ P. Gras, ${ }^{31}$ G. Hamel de Monchenault, ${ }^{31}$ P. Jarry, ${ }^{31}$ C. Leloup, ${ }^{31}$

E. Locci, ${ }^{31}$ J. Malcles, ${ }^{31}$ G. Negro, ${ }^{31}$ J. Rander, ${ }^{31}$ A. Rosowsky, ${ }^{31}$ M. Ö. Sahin,${ }^{31}$ M. Titov, ${ }^{31}$ A. Abdulsalam, ${ }^{32,1}$ C. Amendola, ${ }^{32}$ I. Antropov, ${ }^{32}$ F. Beaudette, ${ }^{32}$ P. Busson, ${ }^{32}$ C. Charlot, ${ }^{32}$ R. Granier de Cassagnac, ${ }^{32}$ I. Kucher, ${ }^{32}$ A. Lobanov, ${ }^{32}$ J. Martin Blanco, ${ }^{32}$ C. Martin Perez, ${ }^{32}$ M. Nguyen, ${ }^{32}$ C. Ochando,${ }^{32}$ G. Ortona, ${ }^{32}$ P. Paganini, ${ }^{32}$ P. Pigard,${ }^{32}$ J. Rembser, ${ }^{32}$ R. Salerno, ${ }^{32}$ J. B. Sauvan, ${ }^{32}$ Y. Sirois, ${ }^{32}$ A. G. Stahl Leiton, ${ }^{32}$ A. Zabi, ${ }^{32}$ A. Zghiche, ${ }^{32}$ J.-L. Agram, ${ }^{33, m}$ J. Andrea, ${ }^{33}$ D. Bloch, ${ }^{33}$ J.-M. Brom, ${ }^{33}$ E. C. Chabert, ${ }^{33}$ V. Cherepanov, ${ }^{33}$ C. Collard ${ }^{33}$ E. Conte, ${ }^{33, m}$ J.-C. Fontaine, ${ }^{33, m}$ D. Gelé, ${ }^{33}$ U. Goerlach, ${ }^{33}$ M. Jansová, ${ }^{33}$ A.-C. Le Bihan, ${ }^{33}$ N. Tonon, ${ }^{33}$ P. Van Hove, ${ }^{33}$ S. Gadrat,${ }^{34}$ S. Beauceron, ${ }^{35}$ C. Bernet, ${ }^{35}$ G. Boudoul,${ }^{35}$ N. Chanon, ${ }^{35}$ R. Chierici, ${ }^{35}$ D. Contardo,${ }^{35}$ P. Depasse,${ }^{35}$ H. El Mamouni,${ }^{35}$ J. Fay, ${ }^{35}$ L. Finco,${ }^{35}$ S. Gascon, ${ }^{35}$ M. Gouzevitch, ${ }^{35}$ G. Grenier, ${ }^{35}$ B. Ille, ${ }^{35}$ F. Lagarde,${ }^{35}$ I. B. Laktineh,${ }^{35}$ H. Lattaud, ${ }^{35}$ M. Lethuillier, ${ }^{35}$ L. Mirabito, ${ }^{35}$ S. Perries, ${ }^{35}$ A. Popov, ${ }^{35, n}$ V. Sordini, ${ }^{35}$ G. Touquet, ${ }^{35}$ M. Vander Donckt, ${ }^{35}$ S. Viret, ${ }^{35}$ T. Toriashvili, ${ }^{36,0}$ Z. Tsamalaidze,${ }^{37, h}$ C. Autermann, ${ }^{38}$ L. Feld, ${ }^{38}$ M. K. Kiesel, ${ }^{38}$ K. Klein, ${ }^{38}$ M. Lipinski,${ }^{38}$ M. Preuten, ${ }^{38}$ M. P. Rauch, ${ }^{38}$ C. Schomakers, ${ }^{38}$ J. Schulz,${ }^{38}$ M. Teroerde, ${ }^{38}$ B. Wittmer, ${ }^{38}$ A. Albert, ${ }^{39}$ D. Duchardt, ${ }^{39}$ M. Erdmann, ${ }^{39}$ S. Erdweg, ${ }^{39}$

T. Esch, ${ }^{39}$ R. Fischer, ${ }^{39}$ S. Ghosh ${ }^{39}$ A. Güth, ${ }^{39}$ T. Hebbeker, ${ }^{39}$ C. Heidemann, ${ }^{39}$ K. Hoepfner, ${ }^{39}$ H. Keller, ${ }^{39}$ L. Mastrolorenzo, ${ }^{39}$ M. Merschmeyer ${ }^{39}$ A. Meyer, ${ }^{39}$ P. Millet, ${ }^{39}$ S. Mukherjee, ${ }^{39}$ T. Pook, ${ }^{39}$ M. Radziej,${ }^{39}$ H. Reithler, ${ }^{39}$ M. Rieger, ${ }^{39}$ A. Schmidt, ${ }^{39}$ D. Teyssier, ${ }^{39}$ S. Thüer, ${ }^{39}$ G. Flügge, ${ }^{40}$ O. Hlushchenko, ${ }^{40}$ T. Kress, ${ }^{40}$ T. Müller,${ }^{40}$ A. Nehrkorn, ${ }^{40}$ A. Nowack, ${ }^{40}$ C. Pistone, ${ }^{40}$ O. Pooth, ${ }^{40}$ D. Roy, ${ }^{40}$ H. Sert, ${ }^{40}$ A. Stahl,${ }^{40, p}$ M. Aldaya Martin, ${ }^{41}$ T. Arndt,${ }^{41}$

C. Asawatangtrakuldee, ${ }^{41}$ I. Babounikau, ${ }^{41}$ K. Beernaert, ${ }^{41}$ O. Behnke, ${ }^{41}$ U. Behrens, ${ }^{41}$ A. Bermúdez Martínez, ${ }^{41}$ D. Bertsche, ${ }^{41}$ A. A. Bin Anuar, ${ }^{41}$ K. Borras,${ }^{41, q}$ V. Botta,${ }^{41}$ A. Campbell, ${ }^{41}$ P. Connor ${ }^{41}$ C. Contreras-Campana, ${ }^{41}$ V. Danilov, ${ }^{41}$ A. De Wit, ${ }^{41}$ M. M. Defranchis,${ }^{41}$ C. Diez Pardos, ${ }^{41}$ D. Domínguez Damiani, ${ }^{41}$ G. Eckerlin, ${ }^{41}$ T. Eichhorn, ${ }^{41}$ A. Elwood, ${ }^{41}$ E. Eren, ${ }^{41}$ E. Gallo, ${ }^{41, r}$ A. Geiser, ${ }^{41}$ J. M. Grados Luyando, ${ }^{41}$ A. Grohsjean, ${ }^{41}$ M. Guthoff, ${ }^{41}$ M. Haranko, ${ }^{41}$ A. Harb ${ }^{41}$ J. Hauk,${ }^{41}$ H. Jung, ${ }^{41}$ M. Kasemann, ${ }^{41}$ J. Keaveney, ${ }^{41}$ C. Kleinwort, ${ }^{41}$ J. Knolle, ${ }^{41}$ D. Krücker, ${ }^{41}$ W. Lange, ${ }^{41}$ A. Lelek, ${ }^{41}$ T. Lenz, ${ }^{41}$ J. Leonard, ${ }^{41}$ K. Lipka, ${ }^{41}$ W. Lohmann, ${ }^{41, s}$ R. Mankel, ${ }^{41}$ I.-A. Melzer-Pellmann, ${ }^{41}$ A. B. Meyer, ${ }^{41}$ M. Meyer, ${ }^{41}$ M. Missiroli, ${ }^{41}$ G. Mittag, ${ }^{41}$ J. Mnich,${ }^{41}$ V. Myronenko, ${ }^{41}$ S. K. Pflitsch, ${ }^{41}$ D. Pitzl, ${ }^{41}$ A. Raspereza, ${ }^{41}$ M. Savitskyi ${ }^{41}$ P. Saxena, ${ }^{41}$ P. Schütze ${ }^{41}$ C. Schwanenberger, ${ }^{41}$ R. Shevchenko, ${ }^{41}$ A. Singh, ${ }^{41}$ H. Tholen,${ }^{41}$ O. Turkot,${ }^{41}$ A. Vagnerini, ${ }^{41}$ G. P. Van Onsem, ${ }^{41}$ R. Walsh, ${ }^{41}$ Y. Wen, ${ }^{41}$ K. Wichmann,${ }^{41}$ C. Wissing, ${ }^{41}$ O. Zenaiev, ${ }^{41}$ R. Aggleton, ${ }^{42}$ S. Bein ${ }^{42}$ L. Benato, ${ }^{42}$ A. Benecke, ${ }^{42}$ V. Blobel, ${ }^{42}$ T. Dreyer, ${ }^{42}$ A. Ebrahimi, ${ }^{42}$ E. Garutti, ${ }^{42}$ D. Gonzalez, ${ }^{42}$ P. Gunnellini ${ }^{42}$ J. Haller, ${ }^{42}$ A. Hinzmann, ${ }^{42}$ A. Karavdina, ${ }^{42}$ G. Kasieczka, ${ }^{42}$ R. Klanner, ${ }^{42}$ R. Kogler, ${ }^{42}$ N. Kovalchuk, ${ }^{42}$ S. Kurz, ${ }^{42}$ V. Kutzner, ${ }^{42}$ J. Lange, ${ }^{42}$ D. Marconi, ${ }^{42}$ J. Multhaup, ${ }^{42}$ M. Niedziela, ${ }^{42}$ C. E. N. Niemeyer, ${ }^{42}$ D. Nowatschin,${ }^{42}$ A. Perieanu, ${ }^{42}$ A. Reimers, ${ }^{42}$ O. Rieger, ${ }^{42}$ C. Scharf, ${ }^{42}$ P. Schleper, ${ }^{42}$ S. Schumann, ${ }^{42}$ J. Schwandt, ${ }^{42}$ J. Sonneveld, ${ }^{42}$ H. Stadie, ${ }^{42}$ G. Steinbrück, ${ }^{42}$ F. M. Stober, ${ }^{42}$ M. Stöver, ${ }^{42}$ A. Vanhoefer, ${ }^{42}$ B. Vormwald, ${ }^{42}$ I. Zoi, ${ }^{42}$ M. Akbiyik, ${ }^{43}$ C. Barth, ${ }^{43}$ M. Baselga, ${ }^{43}$ S. Baur, ${ }^{43}$ E. Butz ${ }^{43}$ R. Caspart, ${ }^{43}$ T. Chwalek, ${ }^{43}$ F. Colombo, ${ }^{43}$ W. De Boer, ${ }^{43}$ A. Dierlamm, ${ }^{43}$ K. El Morabit, ${ }^{43}$ N. Faltermann, ${ }^{43}$ B. Freund, ${ }^{43}$ M. Giffels, ${ }^{43}$ M. A. Harrendorf, ${ }^{43}$ F. Hartmann, ${ }^{43, p}$ S. M. Heindl, ${ }^{43}$ U. Husemann, ${ }^{43}$ I. Katkov, ${ }^{43, n}$ S. Kudella, ${ }^{43}$ S. Mitra, ${ }^{43}$ M. U. Mozer, ${ }^{43}$ Th. Müller, ${ }^{43}$ M. Musich,${ }^{43}$ M. Plagge, ${ }^{43}$ G. Quast,${ }^{43}$ K. Rabbertz ${ }^{43}$ M. Schröder, ${ }^{43}$ I. Shvetsov ${ }^{43}$ H. J. Simonis, ${ }^{43}$ R. Ulrich ${ }^{43}$ S. Wayand, ${ }^{43}$ M. Weber, ${ }^{43}$ T. Weiler, ${ }^{43}$ C. Wöhrmann, ${ }^{43}$ R. Wolf, ${ }^{43}$ G. Anagnostou, ${ }^{44}$ G. Daskalakis, ${ }^{44}$ T. Geralis, ${ }^{44}$ A. Kyriakis, ${ }^{44}$ D. Loukas, ${ }^{44}$ G. Paspalaki, ${ }^{44}$ G. Karathanasis, ${ }^{45}$ P. Kontaxakis, ${ }^{45}$ A. Panagiotou, ${ }^{45}$ I. Papavergou, ${ }^{45}$ N. Saoulidou, ${ }^{45}$ E. Tziaferi, ${ }^{45}$ K. Vellidis, ${ }^{45}$ K. Kousouris, ${ }^{46}$ I. Papakrivopoulos, ${ }^{46}$ G. Tsipolitis, ${ }^{46}$ I. Evangelou, ${ }^{47}$ C. Foudas,${ }^{47}$ P. Gianneios, ${ }^{47}$ P. Katsoulis, ${ }^{47}$ P. Kokkas, ${ }^{47}$ S. Mallios,${ }^{47}$ N. Manthos,${ }^{47}$ I. Papadopoulos,${ }^{47}$ E. Paradas, ${ }^{47}$ J. Strologas, ${ }^{47}$ F. A. Triantis,${ }^{47}$ D. Tsitsonis,${ }^{47}$ M. Bartók, ${ }^{48, t}$ M. Csanad, ${ }^{48}$ N. Filipovic, ${ }^{48}$ P. Major, ${ }^{48}$ M. I. Nagy, ${ }^{48}$ G. Pasztor ${ }^{48}$ O. Surányi, ${ }^{48}$ G. I. Veres, ${ }^{48}$ G. Bencze, ${ }^{49}$ 
C. Hajdu, ${ }^{49}$ D. Horvath, ${ }^{49, u}$ Á. Hunyadi, ${ }^{49}$ F. Sikler, ${ }^{49}$ T. Á. Vámi, ${ }^{49}$ V. Veszpremi, ${ }^{49}$ G. Vesztergombi ${ }^{49, \dagger}$, v N. Beni, ${ }^{50}$ S. Czellar, ${ }^{50}$ J. Karancsi, ${ }^{50, t}$ A. Makovec,${ }^{50}$ J. Molnar, ${ }^{50}$ Z. Szillasi,${ }^{50}$ P. Raics, ${ }^{51}$ Z. L. Trocsanyi, ${ }^{51}$ B. Ujvari, ${ }^{51}$ S. Choudhury, ${ }^{52}$ J. R. Komaragiri, ${ }^{52}$ P. C. Tiwari, ${ }^{52}$ S. Bahinipati, ${ }^{53, w}$ C. Kar, ${ }^{53}$ P. Mal, ${ }^{53}$ K. Mandal, ${ }^{53}$ A. Nayak, ${ }^{53, x}$ D. K. Sahoo, ${ }^{53, w}$ S. K. Swain, ${ }^{53}$ S. Bansal, ${ }^{54}$ S. B. Beri, ${ }^{54}$ V. Bhatnagar, ${ }^{54}$ S. Chauhan, ${ }^{54}$ R. Chawla, ${ }^{54}$ N. Dhingra, ${ }^{54}$ R. Gupta ${ }^{54}$ A. Kaur, ${ }^{54}$ M. Kaur, ${ }^{54}$ S. Kaur,${ }^{54}$ P. Kumari,${ }^{54}$ M. Lohan, ${ }^{54}$ A. Mehta, ${ }^{54}$ K. Sandeep, ${ }^{54}$ S. Sharma, ${ }^{54}$ J. B. Singh, ${ }^{54}$ A. K. Virdi,${ }^{54}$ G. Walia, ${ }^{54}$ A. Bhardwaj, ${ }^{55}$ B. C. Choudhary, ${ }^{55}$ R. B. Garg,${ }^{55}$ M. Gola, ${ }^{55}$ S. Keshri, ${ }^{55}$ Ashok Kumar, ${ }^{55}$ S. Malhotra, ${ }^{55}$ M. Naimuddin, ${ }^{55}$ P. Priyanka, ${ }^{55}$ K. Ranjan,${ }^{55}$ Aashaq Shah ${ }^{55}$ R. Sharma, ${ }^{55}$ R. Bhardwaj, ${ }^{56, y}$ M. Bharti, ${ }^{56, y}$ R. Bhattacharya, ${ }^{56}$ S. Bhattacharya ${ }^{56}$ U. Bhawandeep, ${ }^{56, y}$ D. Bhowmik, ${ }^{56}$ S. Dey, ${ }^{56}$ S. Dutt, ${ }^{56, y}$ S. Dutta,${ }^{56}$ S. Ghosh, ${ }^{56}$ K. Mondal, ${ }^{56}$ S. Nandan, ${ }^{56}$ A. Purohit, ${ }^{56}$ P. K. Rout, ${ }^{56}$ A. Roy, ${ }^{56}$ S. Roy Chowdhury, ${ }^{56}$ G. Saha, ${ }^{56}$ S. Sarkar, ${ }^{56}$ M. Sharan, ${ }^{56}$ B. Singh, ${ }^{56, y}$ S. Thakur, ${ }^{56, y}$ P. K. Behera, ${ }^{57}$ R. Chudasama, ${ }^{58}$ D. Dutta, ${ }^{58}$ V. Jha ${ }^{58}$ V. Kumar, ${ }^{58}$ P. K. Netrakanti, ${ }^{58}$ L. M. Pant, ${ }^{58}$ P. Shukla, ${ }^{58}$ T. Aziz, ${ }^{59}$ M. A. Bhat, ${ }^{59}$ S. Dugad ${ }^{59}$ G. B. Mohanty, ${ }^{59}$ N. Sur, ${ }^{59}$ B. Sutar,${ }^{59}$

Ravindra Kumar Verma ${ }^{50}$ S. Banerjee, ${ }^{60}$ S. Bhattacharya, ${ }^{60}$ S. Chatterjee,${ }^{60}$ P. Das, ${ }^{60}$ M. Guchait,${ }^{60}$ Sa. Jain, ${ }^{60}$ S. Karmakar, ${ }^{60}$ S. Kumar, ${ }^{60}$ M. Maity, ${ }^{60, z}$ G. Majumder, ${ }^{60}$ K. Mazumdar ${ }^{60}$ N. Sahoo, ${ }^{60}$ T. Sarkar, ${ }^{60, z}$ S. Chauhan, ${ }^{61}$ S. Dube ${ }^{61}$ V. Hegde,${ }^{61}$ A. Kapoor, ${ }^{61}$ K. Kothekar, ${ }^{61}$ S. Pandey, ${ }^{61}$ A. Rane, ${ }^{61}$ A. Rastogi, ${ }^{61}$ S. Sharma, ${ }^{61}$ S. Chenarani, ${ }^{62, a a}$ E. Eskandari Tadavani, ${ }^{62}$ S. M. Etesami ${ }^{62, a a}$ M. Khakzad, ${ }^{62}$ M. Mohammadi Najafabadi, ${ }^{62}$ M. Naseri, ${ }^{62}$

F. Rezaei Hosseinabadi, ${ }^{62}$ B. Safarzadeh, ${ }^{62, b b}$ M. Zeinali, ${ }^{62}$ M. Felcini, ${ }^{63}$ M. Grunewald, ${ }^{63}$ M. Abbrescia, ${ }^{64 a, 64 b}$ C. Calabria ${ }^{64 a, 64 b}$ A. Colaleo, ${ }^{64 a}$ D. Creanza,${ }^{64 a, 64 c}$ L. Cristella, ${ }^{64 a, 64 b}$ N. De Filippis, ${ }^{64 a, 64 c}$ M. De Palma,${ }^{64 a, 64 b}$ A. Di Florio ${ }^{64 a, 64 b}$ F. Errico, ${ }^{64 a, 64 b}$ L. Fiore ${ }^{64 a}$ A. Gelmi, ${ }^{64 a, 64 b}$ G. Iaselli, ${ }^{64 a, 64 c}$ M. Ince, ${ }^{64 a, 64 b}$ S. Lezki, ${ }^{64 a, 64 b}$ G. Maggi ${ }^{64 a, 64 c}$ M. Maggi, ${ }^{64 a}$ G. Miniello, ${ }^{64 a, 64 b}$ S. My, ${ }^{64 a, 64 b}$ S. Nuzzo, ${ }^{64 a, 64 b}$ A. Pompili, ${ }^{64 a, 64 b}$ G. Pugliese,${ }^{64 a, 64 c}$ R. Radogna, ${ }^{64 a}$ A. Ranieri, ${ }^{64 a}$ G. Selvaggi, ${ }^{64 a, 64 b}$ A. Sharma ${ }^{64 a}$ L. Silvestris, ${ }^{64 a}$ R. Venditti, ${ }^{64 a}$ P. Verwilligen, ${ }^{64 a}$ G. Zito, ${ }^{64 a}$ G. Abbiendi, ${ }^{65 a}$ C. Battilana, ${ }^{65 a, 65 b}$ D. Bonacorsi, ${ }^{65 a, 65 b}$ L. Borgonovi ${ }^{65 a, 65 b}$ S. Braibant-Giacomelli, ${ }^{65 a, 65 b}$ R. Campanini, ${ }^{65 a, 65 b}$ P. Capiluppi, ${ }^{65 a, 65 b}$ A. Castro, ${ }^{65 a, 65 b}$ F. R. Cavallo ${ }^{65 a}$ S. S. Chhibra, ${ }^{65 a, 65 b}$ C. Ciocca, ${ }^{65 a}$ G. Codispoti, ${ }^{65 a, 65 b}$ M. Cuffiani, ${ }^{65 a, 65 b}$ G. M. Dallavalle, ${ }^{65 a}$ F. Fabbri, ${ }^{65 a}$ A. Fanfani, ${ }^{65 a, 65 b}$ E. Fontanesi, ${ }^{65 a}$ P. Giacomelli, ${ }^{65 a}$ C. Grandi, ${ }^{65 a}$ L. Guiducci, ${ }^{65 a, 65 b}$ S. Lo Meo, ${ }^{65 a}$ S. Marcellini, ${ }^{65 a}$ G. Masetti, ${ }^{65 a}$ A. Montanari, ${ }^{65 a}$ F. L. Navarria, ${ }^{65,65 b}$ A. Perrotta, ${ }^{65 a}$ F. Primavera, ${ }^{65 a, 65 b, p}$ A. M. Rossi ${ }^{65 a, 65 b}$ T. Rovelli, ${ }^{65 a, 65 b}$ G. P. Siroli ${ }^{65 a, 65 b}$ N. Tosi ${ }^{65 a}$ S. Albergo, ${ }^{66 a, 66 b}$ A. Di Mattia ${ }^{66 a}$ R. Potenza, ${ }^{66 a, 66 b}$ A. Tricomi, ${ }^{66 a, 66 b}$ C. Tuve, ${ }^{66 a, 66 b}$ G. Barbagli, ${ }^{67 a}$ K. Chatterjee, ${ }^{67 a, 67 b}$ V. Ciulli, ${ }^{67,67 b}$ C. Civinini, ${ }^{67 a}$ R. D'Alessandro, ${ }^{67 a, 67 b}$ E. Focardi ${ }^{67 a, 67 b}$ G. Latino, ${ }^{67 a}$ P. Lenzi, ${ }^{67 a, 67 b}$ M. Meschini, ${ }^{67 a}$ S. Paoletti, ${ }^{67 a}$ L. Russo, ${ }^{67 a, c c}$ G. Sguazzoni, ${ }^{67 a}$ D. Strom, ${ }^{67 a}$

L. Viliani, ${ }^{67 a}$ L. Benussi, ${ }^{68}$ S. Bianco, ${ }^{68}$ F. Fabbri, ${ }^{68}$ D. Piccolo,${ }^{68}$ F. Ferro, ${ }^{69 a}$ R. Mulargia, ${ }^{6 a, 69 b}$ F. Ravera, ${ }^{69 a, 69 b}$ E. Robutti, ${ }^{69 \mathrm{a}}$ S. Tosi, ${ }^{69 a, 69 \mathrm{~b}}$ A. Benaglia, ${ }^{70 \mathrm{a}}$ A. Beschi, ${ }^{70 a, 70 \mathrm{~b}}$ F. Brivio, ${ }^{70 a, 70 b}$ V. Ciriolo, ${ }^{70 a, 70 b, p}$ S. Di Guida, ${ }^{70 a, 70 b, p}$ M. E. Dinardo, ${ }^{70 a, 70 b}$ S. Fiorendi, ${ }^{70 a, 70 b}$ S. Gennai, ${ }^{70 a}$ A. Ghezzi, ${ }^{70 a, 70 b}$ P. Govoni, ${ }^{70 a, 70 b}$ M. Malberti, ${ }^{70 a, 70 b}$ S. Malvezzi, ${ }^{70 a}$ A. Massironi, ${ }^{70 a, 70 b}$ D. Menasce, ${ }^{70 a}$ F. Monti, ${ }^{70 a}$ L. Moroni, ${ }^{70 a}$ M. Paganoni, ${ }^{70 a, 70 b}$ D. Pedrini, ${ }^{70 a}$ S. Ragazzi, ${ }^{70 a, 70 b}$ T. Tabarelli de Fatis, ${ }^{70 a, 70 b}$ D. Zuolo, ${ }^{70 a, 70 b}$ S. Buontempo ${ }^{71 a}$ N. Cavallo, ${ }^{71 a, 71 c}$ A. De Iorio, ${ }^{71 a, 71 b}$ A. Di Crescenzo, ${ }^{71 a, 71 b}$ F. Fabozzi, ${ }^{71 a, 71 c}$ F. Fienga, ${ }^{71 a}$ G. Galati, ${ }^{71 a}$ A. O. M. Iorio,${ }^{71 a, 71 b}$ W. A. Khan, ${ }^{71 a}$ L. Lista, ${ }^{71 a}$ S. Meola, ${ }^{71 a, 71 d, p}$ P. Paolucci, ${ }^{71 a, p}$ C. Sciacca, ${ }^{71 a, 71 b}$ E. Voevodina,${ }^{71 a, 71 b}$ P. Azzi, ${ }^{72 a}$ N. Bacchetta, ${ }^{72 a}$ D. Bisello, ${ }^{72 a, 72 b}$ A. Boletti, ${ }^{72 a, 72 b}$ A. Bragagnolo, ${ }^{72 a}$

R. Carlin, ${ }^{72 a, 72 b}$ P. Checchia, ${ }^{72 a}$ M. Dall'Osso, ${ }^{72 a, 72 b}$ P. De Castro Manzano, ${ }^{72 a}$ T. Dorigo, ${ }^{72 a}$ U. Dosselli, ${ }^{72 a}$

F. Gasparini, ${ }^{72 a, 72 b}$ U. Gasparini, ${ }^{72 a, 72 b}$ A. Gozzelino, ${ }^{72 a}$ S. Y. Hoh, ${ }^{72 a}$ S. Lacaprara, ${ }^{72 a}$ P. Lujan, ${ }^{72 a}$ M. Margoni, ${ }^{72 a, 72 b}$ A. T. Meneguzzo, ${ }^{72 a, 72 b}$ J. Pazzini, ${ }^{72 a, 72 b}$ P. Ronchese, ${ }^{72 a, 72 b}$ R. Rossin, ${ }^{72 a, 72 b}$ F. Simonetto, ${ }^{72 a, 72 b}$ A. Tiko, ${ }^{72 a}$ E. Torassa, ${ }^{72 a}$ M. Tosi, ${ }^{72 a, 72 b}$ M. Zanetti, ${ }^{72 a, 72 b}$ P. Zotto, ${ }^{72 a, 72 b}$ G. Zumerle, ${ }^{72 a, 72 b}$ A. Braghieri, ${ }^{73 a}$ A. Magnani, ${ }^{73 a}$ P. Montagna, ${ }^{73 a, 73 b}$ S. P. Ratti, ${ }^{73 a, 73 b}$ V. Re, ${ }^{73 a}$ M. Ressegotti, ${ }^{73 a, 73 b}$ C. Riccardi, ${ }^{73 a, 73 b}$ P. Salvini, ${ }^{73 a}$ I. Vai, ${ }^{73 a, 73 b}$ P. Vitulo, ${ }^{73 a, 73 b}$ M. Biasini, ${ }^{74 a, 74 b}$ G. M. Bilei, ${ }^{74 a}$ C. Cecchi, ${ }^{74 a, 74 b}$ D. Ciangottini, ${ }^{74 a}{ }^{74 b}$ L. Fanò, ${ }^{74 a, 74 b}$ P. Lariccia, ${ }^{74 a, 74 b}$ R. Leonardi, ${ }^{74 a, 74 b}$ E. Manoni, ${ }^{74 a}$ G. Mantovani, ${ }^{74 a, 74 b}$ V. Mariani, ${ }^{74 a, 74 b}$ M. Menichelli, ${ }^{74 a}$ A. Rossi, ${ }^{74,74 b}$ A. Santocchia, ${ }^{74 a, 74 b}$ D. Spiga, ${ }^{74 a}$ K. Androsov, ${ }^{75 a}$ P. Azzurri, ${ }^{75 a}$ G. Bagliesi, ${ }^{75 a}$ L. Bianchini, ${ }^{75 a}$ T. Boccali, ${ }^{75 a}$ L. Borrello, ${ }^{75 a}$ R. Castaldi, ${ }^{75 a}$ M. A. Ciocci, ${ }^{75 a}, 75 b$ R. Dell'Orso, ${ }^{75 a}$ G. Fedi, ${ }^{75 a}$ F. Fiori, ${ }^{75 a, 75 c}$ L. Giannini, ${ }^{75 a, 75 c}$ A. Giassi, ${ }^{75 a}$ M. T. Grippo, ${ }^{75 a}$ F. Ligabue, ${ }^{75 a, 75 c}$ E. Manca, ${ }^{75 a, 75 c}$ G. Mandorli, ${ }^{75 a, 75 c}$ A. Messineo, ${ }^{75 a, 75 b}$ F. Palla ${ }^{75 a}$ A. Rizzi, ${ }^{75 a, 75 b}$ G. Rolandi,${ }^{75 a, d d}$ P. Spagnolo, ${ }^{75 a}$ R. Tenchini, ${ }^{75 a}$ G. Tonelli, ${ }^{75 a, 75 b}$ A. Venturi, ${ }^{75 a}$ P. G. Verdini, ${ }^{75 a}$ L. Barone,${ }^{76 a, 76 b}$ F. Cavallari, ${ }^{76 a}$ M. Cipriani, ${ }^{76 a, 76 b}$ D. Del Re, ${ }^{76 a, 76 b}$ E. Di Marco, ${ }^{76 a, 76 b}$ M. Diemoz, ${ }^{76 a}$ S. Gelli, ${ }^{76 a, 76 b}$ E. Longo, ${ }^{76 a, 76 b}$ B. Marzocchi, ${ }^{76 a, 76 b}$ P. Meridiani ${ }^{76 a}$ G. Organtini, ${ }^{76 a, 76 b}$ F. Pandolfi, ${ }^{76 a}$ R. Paramatti, ${ }^{76 a, 76 b}$ F. Preiato, ${ }^{76 a, 76 b}$ S. Rahatlou, ${ }^{76 a, 76 b}$ C. Rovelli, ${ }^{76 a}$ F. Santanastasio, ${ }^{76 a, 76 b}$ N. Amapane, ${ }^{77 a, 77 b}$ R. Arcidiacono, ${ }^{77 a, 77 c}$ S. Argiro, ${ }^{77 a, 77 b}$ M. Arneodo, ${ }^{77 a, 77 c}$ N. Bartosik, ${ }^{77 a}$ R. Bellan, ${ }^{77 a, 77 b}$ C. Biino, ${ }^{77 a}$ A. Cappati, ${ }^{77 a, 77 b}$ N. Cartiglia, ${ }^{77 a}$ F. Cenna, ${ }^{77 a, 77 b}$ S. Cometti, ${ }^{77 a}$ M. Costa, ${ }^{77 a, 77 b}$ R. Covarelli, ${ }^{77 a, 77 b}$ N. Demaria, ${ }^{77 a}$ B. Kiani, ${ }^{77 a, 77 b}$ 
C. Mariotti, ${ }^{77 a}$ S. Maselli, ${ }^{77 a}$ E. Migliore, ${ }^{77 a, 77 b}$ V. Monaco, ${ }^{77 a, 77 b}$ E. Monteil, ${ }^{77 a, 77 b}$ M. Monteno, ${ }^{77 a}$ M. M. Obertino, ${ }^{77 a, 77 b}$ L. Pacher, ${ }^{77 a, 77 b}$ N. Pastrone, ${ }^{77 a}$ M. Pelliccioni, ${ }^{77 a}$ G. L. Pinna Angioni, ${ }^{77 a, 77 b}$ A. Romero, ${ }^{77 a, 77 b}$ M. Ruspa, ${ }^{77 a, 77 c}$ R. Sacchi, ${ }^{77 a, 77 b}$ R. Salvatico, ${ }^{77 a, 77 b}$ K. Shchelina, ${ }^{77 a, 77 b}$ V. Sola, ${ }^{77 a}$ A. Solano, ${ }^{77 a, 77 b}$ D. Soldi, ${ }^{77 a, 77 b}$ A. Staiano, ${ }^{77 a}$ S. Belforte, ${ }^{78 a}$ V. Candelise, ${ }^{78 a, 78 b}$ M. Casarsa, ${ }^{78 a}$ F. Cossutti, ${ }^{78 a}$ A. Da Rold, ${ }^{78 a, 78 b}$ G. Della Ricca, ${ }^{78 a, 78 b}$ F. Vazzoler, ${ }^{78 a, 78 b}$ A. Zanetti, ${ }^{78 a}$ D. H. Kim, ${ }^{79}$ G. N. Kim, ${ }^{79}$ M. S. Kim, ${ }^{79}$ J. Lee, ${ }^{79}$ S. Lee ${ }^{79}$ S. W. Lee, ${ }^{79}$ C. S. Moon, ${ }^{79}$ Y. D. Oh, ${ }^{79}$ S. I. Pak, ${ }^{79}$ S. Sekmen, ${ }^{79}$ D. C. Son,${ }^{79}$ Y. C. Yang,${ }^{79}$ H. Kim, ${ }^{80}$ D. H. Moon,${ }^{80}$ G. Oh, ${ }^{80}$ B. Francois,${ }^{81}$ J. Goh,${ }^{81, e e}$ T. J. Kim, ${ }^{81}$ S. Cho, ${ }^{82}$ S. Choi ${ }^{82}$ Y. Go, ${ }^{82}$ D. Gyun ${ }^{82}$ S. Ha ${ }^{82}$ B. Hong, ${ }^{82}$ Y. Jo, ${ }^{82}$ K. Lee, ${ }^{82}$ K. S. Lee, ${ }^{82}$ S. Lee, ${ }^{82}$ J. Lim, ${ }^{82}$ S. K. Park, ${ }^{82}$ Y. Roh, ${ }^{82}$ H. S. Kim, ${ }^{83}$ J. Almond ${ }^{84}$ J. Kim, ${ }^{84}$ J. S. Kim ${ }^{84}$ H. Lee, ${ }^{84}$ K. Lee, ${ }^{84}$ K. Nam,${ }^{84}$ S. B. Oh, ${ }^{84}$ B. C. Radburn-Smith, ${ }^{84}$ S. h. Seo, ${ }^{84}$ U. K. Yang,${ }^{84}$ H. D. Yoo,${ }^{84}$ G. B. Yu ${ }^{84}$ D. Jeon ${ }^{85}$ H. Kim,${ }^{85}$ J. H. Kim, ${ }^{85}$ J. S. H. Lee, ${ }^{85}$ I. C. Park,${ }^{85}$ Y. Choi ${ }^{86}$ C. Hwang, ${ }^{86}$ J. Lee ${ }^{86}$ I. Yu, ${ }^{86}$ V. Dudenas, ${ }^{87}$ A. Juodagalvis, ${ }^{87}$ J. Vaitkus, ${ }^{87}$ I. Ahmed, ${ }^{88}$ Z. A. Ibrahim, ${ }^{88}$ M. A. B. Md Ali, ${ }^{88, f f}$ F. Mohamad Idris, ${ }^{88, g g}$ W. A. T. Wan Abdullah, ${ }^{88}$ M. N. Yusli, ${ }^{88}$ Z. Zolkapli, ${ }^{88}$ J. F. Benitez, ${ }^{89}$ A. Castaneda Hernandez, ${ }^{89}$ J. A. Murillo Quijada, ${ }^{89}$ H. Castilla-Valdez,${ }^{90}$ E. De La Cruz-Burelo,${ }^{90}$ M. C. Duran-Osuna,${ }^{90}$ I. Heredia-De La Cruz, ${ }^{90, \text { hh }}$ R. Lopez-Fernandez, ${ }^{90}$ J. Mejia Guisao, ${ }^{90}$ R. I. Rabadan-Trejo, ${ }^{90}$ M. Ramirez-Garcia, ${ }^{90}$ G. Ramirez-Sanchez, ${ }^{90}$ R. Reyes-Almanza, ${ }^{90}$ A. Sanchez-Hernandez, ${ }^{90}$ S. Carrillo Moreno, ${ }^{91}$ C. Oropeza Barrera, ${ }^{91}$ F. Vazquez Valencia, ${ }^{91}$ J. Eysermans, ${ }^{92}$ I. Pedraza, ${ }^{92}$ H. A. Salazar Ibarguen, ${ }^{92}$ C. Uribe Estrada, ${ }^{92}$ A. Morelos Pineda, ${ }^{93}$ D. Krofcheck, ${ }^{94}$ S. Bheesette, ${ }^{95}$ P. H. Butler, ${ }^{95}$ A. Ahmad, ${ }^{96}$ M. Ahmad, ${ }^{96}$ M. I. Asghar, ${ }^{96}$ Q. Hassan, ${ }^{96}$ H. R. Hoorani, ${ }^{96}$ A. Saddique, ${ }^{96}$ M. A. Shah, ${ }^{96}$ M. Shoaib, ${ }^{96}$ M. Waqas, ${ }^{96}$ H. Bialkowska, ${ }^{97}$ M. Bluj, ${ }^{97}$ B. Boimska, ${ }^{97}$ T. Frueboes, ${ }^{97}$ M. Górski, ${ }^{97}$ M. Kazana, ${ }^{97}$ M. Szleper, ${ }^{97}$ P. Traczyk ${ }^{97}$ P. Zalewski, ${ }^{97}$ K. Bunkowski, ${ }^{98}$ A. Byszuk, ${ }^{98, i i}$ K. Doroba, ${ }^{98}$ A. Kalinowski, ${ }^{98}$ M. Konecki, ${ }^{98}$ J. Krolikowski, ${ }^{98}$ M. Misiura, ${ }^{98}$ M. Olszewski, ${ }^{98}$ A. Pyskir, ${ }^{98}$ M. Walczak, ${ }^{98}$ M. Araujo, ${ }^{99}$ P. Bargassa, ${ }^{99}$ C. Beirão Da Cruz E Silva, ${ }^{99}$ A. Di Francesco, ${ }^{99}$ P. Faccioli, ${ }^{99}$ B. Galinhas, ${ }^{99}$ M. Gallinaro, ${ }^{99}$ J. Hollar, ${ }^{99}$ N. Leonardo, ${ }^{99}$ J. Seixas, ${ }^{99}$ G. Strong, ${ }^{99}$ O. Toldaiev, ${ }^{99}$ J. Varela, ${ }^{99}$ S. Afanasiev, ${ }^{100}$ P. Bunin, ${ }^{100}$ M. Gavrilenko, ${ }^{100}$ I. Golutvin, ${ }^{100}$

I. Gorbunov, ${ }^{100}$ A. Kamenev, ${ }^{100}$ V. Karjavine, ${ }^{100}$ A. Lanev, ${ }^{100}$ A. Malakhov, ${ }^{100}$ V. Matveev, ${ }^{100, j j, k k}$ P. Moisenz, ${ }^{100}$ V. Palichik, ${ }^{100}$ V. Perelygin, ${ }^{100}$ S. Shmatov, ${ }^{100}$ S. Shulha, ${ }^{100}$ N. Skatchkov, ${ }^{100}$ V. Smirnov, ${ }^{100}$ N. Voytishin, ${ }^{100}$ A. Zarubin, ${ }^{100}$ V. Golovtsov, ${ }^{101}$ Y. Ivanov, ${ }^{101}$ V. Kim, ${ }^{101,11}$ E. Kuznetsova, ${ }^{101, \mathrm{~mm}}$ P. Levchenko, ${ }^{101}$ V. Murzin, ${ }^{101}$ V. Oreshkin, ${ }^{101}$ I. Smirnov, ${ }^{101}$ D. Sosnov, ${ }^{101}$ V. Sulimov, ${ }^{101}$ L. Uvarov, ${ }^{101}$ S. Vavilov, ${ }^{101}$ A. Vorobyev,${ }^{101}$ Yu. Andreev, ${ }^{102}$ A. Dermenev, ${ }^{102}$ S. Gninenko, ${ }^{102}$ N. Golubev, ${ }^{102}$ A. Karneyeu, ${ }^{102}$ M. Kirsanov, ${ }^{102}$ N. Krasnikov, ${ }^{102}$ A. Pashenkov, ${ }^{102}$ D. Tlisov, ${ }^{102}$ A. Toropin, ${ }^{102}$ V. Epshteyn, ${ }^{103}$ V. Gavrilov, ${ }^{103}$ N. Lychkovskaya, ${ }^{103}$ V. Popov,${ }^{103}$ I. Pozdnyakov, ${ }^{103}$ G. Safronov, ${ }^{103}$ A. Spiridonov, ${ }^{103}$ A. Stepennov ${ }^{103}$ V. Stolin, ${ }^{103}$ M. Toms, ${ }^{103}$ E. Vlasov, ${ }^{103}$ A. Zhokin, ${ }^{103}$ T. Aushev, ${ }^{104}$ R. Chistov, ${ }^{105, n n}$ M. Danilov, ${ }^{105, \mathrm{nn}}$ P. Parygin, ${ }^{105}$ D. Philippov, ${ }^{105}$ S. Polikarpov, ${ }^{105, \mathrm{nn}}$ E. Tarkovskii, ${ }^{105}$ V. Andreev, ${ }^{106}$ M. Azarkin, ${ }^{106}$

I. Dremin, ${ }^{106, k \mathrm{kk}}$ M. Kirakosyan, ${ }^{106}$ A. Terkulov, ${ }^{106}$ A. Baskakov, ${ }^{107}$ A. Belyaev,${ }^{107}$ E. Boos, ${ }^{107}$ M. Dubinin, ${ }^{107,00}$ L. Dudko, ${ }^{107}$ A. Ershov, ${ }^{107}$ A. Gribushin, ${ }^{107}$ V. Klyukhin, ${ }^{107}$ O. Kodolova, ${ }^{107}$ I. Lokhtin, ${ }^{107}$ I. Miagkov, ${ }^{107}$ S. Obraztsov, ${ }^{107}$ S. Petrushanko, ${ }^{107}$ V. Savrin, ${ }^{107}$ A. Snigirev, ${ }^{107}$ A. Barnyakov, ${ }^{108, p p}$ V. Blinov, ${ }^{108, p p}$ T. Dimova, ${ }^{108, p p}$ L. Kardapoltsev, ${ }^{108, p p}$ Y. Skovpen, ${ }^{108, p p}$ I. Azhgirey, ${ }^{109}$ I. Bayshev, ${ }^{109}$ S. Bitioukov, ${ }^{109}$ D. Elumakhov, ${ }^{109}$ A. Godizov, ${ }^{109}$ V. Kachanov, ${ }^{109}$ A. Kalinin, ${ }^{109}$ D. Konstantinov, ${ }^{109}$ P. Mandrik, ${ }^{109}$ V. Petrov, ${ }^{109}$ R. Ryutin, ${ }^{109}$ S. Slabospitskii, ${ }^{109}$ A. Sobol, ${ }^{109}$ S. Troshin, ${ }^{109}$ N. Tyurin, ${ }^{109}$ A. Uzunian, ${ }^{109}$ A. Volkov, ${ }^{109}$ A. Babaev,${ }^{110}$ S. Baidali, ${ }^{110}$ V. Okhotnikov, ${ }^{110}$ P. Adzic, ${ }^{11, q q}$ P. Cirkovic, ${ }^{111}$ D. Devetak, ${ }^{111}$ M. Dordevic, ${ }^{111}$ J. Milosevic, ${ }^{111}$ J. Alcaraz Maestre, ${ }^{112}$ A. Álvarez Fernández, ${ }^{112}$ I. Bachiller, ${ }^{112}$ M. Barrio Luna, ${ }^{112}$ J. A. Brochero Cifuentes, ${ }^{112}$ M. Cerrada,${ }^{112}$ N. Colino, ${ }^{112}$ B. De La Cruz, ${ }^{112}$ A. Delgado Peris, ${ }^{112}$ C. Fernandez Bedoya, ${ }^{112}$ J. P. Fernández Ramos, ${ }^{112}$ J. Flix, ${ }^{112}$ M. C. Fouz, ${ }^{112}$ O. Gonzalez Lopez, ${ }^{112}$ S. Goy Lopez, ${ }^{112}$ J. M. Hernandez, ${ }^{112}$ M. I. Josa, ${ }^{112}$ D. Moran, ${ }^{112}$ A. Pérez-Calero Yzquierdo, ${ }^{112}$ J. Puerta Pelayo, ${ }^{112}$ I. Redondo, ${ }^{112}$ L. Romero, ${ }^{112}$ M. S. Soares, ${ }^{112}$ A. Triossi, ${ }^{112}$ C. Albajar, ${ }^{113}$ J. F. de Trocóniz, ${ }^{113}$ J. Cuevas, ${ }^{114}$ C. Erice,${ }^{114}$

J. Fernandez Menendez, ${ }^{114}$ S. Folgueras, ${ }^{114}$ I. Gonzalez Caballero, ${ }^{114}$ J. R. González Fernández, ${ }^{114}$ E. Palencia Cortezon, ${ }^{114}$ V. Rodríguez Bouza, ${ }^{114}$ S. Sanchez Cruz, ${ }^{114}$ P. Vischia, ${ }^{114}$ J. M. Vizan Garcia, ${ }^{114}$ I. J. Cabrillo, ${ }^{115}$ A. Calderon, ${ }^{115}$

B. Chazin Quero, ${ }^{115}$ J. Duarte Campderros, ${ }^{115}$ M. Fernandez ${ }^{115}$ P. J. Fernández Manteca, ${ }^{115}$ A. García Alonso, ${ }^{115}$ J. Garcia-Ferrero, ${ }^{115}$ G. Gomez, ${ }^{115}$ A. Lopez Virto, ${ }^{115}$ J. Marco,${ }^{115}$ C. Martinez Rivero, ${ }^{115}$ P. Martinez Ruiz del Arbol, ${ }^{115}$ F. Matorras, ${ }^{115}$ J. Piedra Gomez, ${ }^{115}$ C. Prieels, ${ }^{115}$ T. Rodrigo, ${ }^{115}$ A. Ruiz-Jimeno, ${ }^{115}$ L. Scodellaro, ${ }^{115}$ N. Trevisani, ${ }^{115}$ I. Vila, ${ }^{115}$ R. Vilar Cortabitarte, ${ }^{115}$ N. Wickramage, ${ }^{116}$ D. Abbaneo, ${ }^{117}$ B. Akgun, ${ }^{117}$ E. Auffray, ${ }^{117}$ G. Auzinger, ${ }^{117}$ P. Baillon, ${ }^{117}$ A. H. Ball, ${ }^{117}$ D. Barney, ${ }^{117}$ J. Bendavid, ${ }^{117}$ M. Bianco, ${ }^{117}$ A. Bocci,${ }^{117}$ C. Botta, ${ }^{117}$ E. Brondolin, ${ }^{117}$ T. Camporesi, ${ }^{117}$ M. Cepeda, ${ }^{117}$ G. Cerminara, ${ }^{117}$ E. Chapon, ${ }^{117}$ Y. Chen, ${ }^{117}$ G. Cucciati, ${ }^{117}$ D. d'Enterria,,${ }^{117}$

A. Dabrowski, ${ }^{117}$ N. Daci, ${ }^{117}$ V. Daponte, ${ }^{117}$ A. David, ${ }^{117}$ A. De Roeck, ${ }^{117}$ N. Deelen, ${ }^{117}$ M. Dobson, ${ }^{117}$ M. Dünser, ${ }^{117}$ 
N. Dupont, ${ }^{117}$ A. Elliott-Peisert, ${ }^{117}$ P. Everaerts,${ }^{117}$ F. Fallavollita, ${ }^{117, \text { rr }}$ D. Fasanella,${ }^{117}$ G. Franzoni, ${ }^{117}$ J. Fulcher, ${ }^{117}$ W. Funk, ${ }^{117}$ D. Gigi, ${ }^{117}$ A. Gilbert, ${ }^{117}$ K. Gill, ${ }^{117}$ F. Glege,${ }^{117}$ M. Gruchala, ${ }^{117}$ M. Guilbaud, ${ }^{117}$ D. Gulhan, ${ }^{117}$ J. Hegeman, ${ }^{117}$ C. Heidegger, ${ }^{117}$ V. Innocente, ${ }^{117}$ A. Jafari, ${ }^{117}$ P. Janot, ${ }^{117}$ O. Karacheban, ${ }^{117, s}$ J. Kieseler, ${ }^{117}$ A. Kornmayer, ${ }^{117}$ M. Krammer, ${ }^{117, a}$ C. Lange, ${ }^{117}$ P. Lecoq ${ }^{117}$ C. Lourenço, ${ }^{117}$ L. Malgeri, ${ }^{117}$ M. Mannelli, ${ }^{117}$ F. Meijers, ${ }^{117}$ J. A. Merlin, ${ }^{117}$ S. Mersi, ${ }^{117}$ E. Meschi, ${ }^{117}$ P. Milenovic, ${ }^{117, \text { ss }}$ F. Moortgat,${ }^{117}$ M. Mulders, ${ }^{117}$ J. Ngadiuba, ${ }^{117}$ S. Nourbakhsh, ${ }^{117}$

S. Orfanelli, ${ }^{117}$ L. Orsini, ${ }^{117}$ F. Pantaleo, ${ }^{117, p}$ L. Pape,${ }^{117}$ E. Perez,${ }^{117}$ M. Peruzzi, ${ }^{117}$ A. Petrilli, ${ }^{117}$ G. Petrucciani, ${ }^{117}$ A. Pfeiffer, ${ }^{117}$ M. Pierini, ${ }^{117}$ F. M. Pitters,${ }^{117}$ D. Rabady,${ }^{117}$ A. Racz, ${ }^{117}$ T. Reis, ${ }^{117}$ M. Rovere, ${ }^{117}$ H. Sakulin, ${ }^{117}$ C. Schäfer, ${ }^{117}$ C. Schwick, ${ }^{117}$ M. Seidel, ${ }^{117}$ M. Selvaggi, ${ }^{117}$ A. Sharma, ${ }^{117}$ P. Silva, ${ }^{117}$ P. Sphicas, ${ }^{117, \text { tt }}$ A. Stakia, ${ }^{117}$ J. Steggemann, ${ }^{117}$

D. Treille, ${ }^{117}$ A. Tsirou, ${ }^{117}$ V. Veckalns, ${ }^{117, \text { uu }}$ M. Verzetti, ${ }^{117}$ W. D. Zeuner, ${ }^{117}$ L. Caminada, ${ }^{118, v v}$ K. Deiters, ${ }^{118}$ W. Erdmann, ${ }^{118}$ R. Horisberger, ${ }^{118}$ Q. Ingram, ${ }^{118}$ H. C. Kaestli, ${ }^{118}$ D. Kotlinski, ${ }^{118}$ U. Langenegger, ${ }^{18}$ T. Rohe, ${ }^{118}$ S. A. Wiederkehr, ${ }^{118}$ M. Backhaus, ${ }^{119}$ L. Bäni, ${ }^{119}$ P. Berger, ${ }^{119}$ N. Chernyavskaya, ${ }^{119}$ G. Dissertori, ${ }^{119}$ M. Dittmar, ${ }^{119}$ M. Donegà ${ }^{119}$ C. Dorfer, ${ }^{119}$ T. A. Gómez Espinosa, ${ }^{119}$ C. Grab, ${ }^{119}$ D. Hits, ${ }^{119}$ T. Klijnsma, ${ }^{119}$ W. Lustermann, ${ }^{119}$ R. A. Manzoni, ${ }^{119}$ M. Marionneau, ${ }^{119}$ M. T. Meinhard,${ }^{119}$ F. Micheli, ${ }^{119}$ P. Musella, ${ }^{119}$ F. Nessi-Tedaldi, ${ }^{119}$ J. Pata, ${ }^{119}$ F. Pauss, ${ }^{119}$ G. Perrin,${ }^{119}$ L. Perrozzi, ${ }^{119}$ S. Pigazzini, ${ }^{119}$ M. Quittnat, ${ }^{119}$ C. Reissel,${ }^{119}$ D. Ruini, ${ }^{119}$ D. A. Sanz Becerra, ${ }^{119}$ M. Schönenberger, ${ }^{119}$ L. Shchutska, ${ }^{119}$ V. R. Tavolaro, ${ }^{119}$ K. Theofilatos, ${ }^{119}$ M. L. Vesterbacka Olsson, ${ }^{119}$ R. Wallny, ${ }^{119}$ D. H. Zhu, ${ }^{119}$ T. K. Aarrestad, ${ }^{120}$ C. Amsler, ${ }^{120, w w}$ D. Brzhechko, ${ }^{120}$ M. F. Canelli, ${ }^{120}$ A. De Cosa, ${ }^{120}$ R. Del Burgo, ${ }^{120}$ S. Donato, ${ }^{120}$ C. Galloni, ${ }^{120}$ T. Hreus, ${ }^{120}$ B. Kilminster, ${ }^{120}$ S. Leontsinis, ${ }^{120}$ I. Neutelings, ${ }^{120}$ G. Rauco, ${ }^{120}$ P. Robmann, ${ }^{120}$ D. Salerno, ${ }^{120}$ K. Schweiger, ${ }^{120}$ C. Seitz, ${ }^{120}$ Y. Takahashi, ${ }^{120}$ A. Zucchetta, ${ }^{120}$ T. H. Doan, ${ }^{121}$ R. Khurana, ${ }^{121}$ C. M. Kuo, ${ }^{121}$ W. Lin, ${ }^{121}$ A. Pozdnyakov, ${ }^{121}$ S. S. Yu, ${ }^{121}$ P. Chang, ${ }^{122}$ Y. Chao, ${ }^{122}$ K. F. Chen, ${ }^{122}$ P. H. Chen, ${ }^{122}$ W.-S. Hou, ${ }^{122}$ Arun Kumar, ${ }^{122}$ Y. F. Liu, ${ }^{122}$ R.-S. Lu, ${ }^{122}$ E. Paganis, ${ }^{122}$ A. Psallidas, ${ }^{122}$ A. Steen, ${ }^{122}$ B. Asavapibhop, ${ }^{123}$ N. Srimanobhas, ${ }^{123}$ N. Suwonjandee, ${ }^{123}$ M. N. Bakirci, ${ }^{124, x x}$ A. Bat, ${ }^{124}$ F. Boran, ${ }^{124}$ S. Damarseckin, ${ }^{124}$ Z. S. Demiroglu, ${ }^{124}$ F. Dolek, ${ }^{124}$ C. Dozen, ${ }^{124}$ E. Eskut, ${ }^{124}$ S. Girgis, ${ }^{124}$ G. Gokbulut, ${ }^{124}$ Y. Guler, ${ }^{124}$ E. Gurpinar, ${ }^{124}$ I. Hos, ${ }^{124, y y}$ C. Isik, ${ }^{124}$ E. E. Kangal, ${ }^{124, z z}$ O. Kara, ${ }^{124}$ U. Kiminsu, ${ }^{124}$ M. Oglakci, ${ }^{124}$ G. Onengut, ${ }^{124}$ K. Ozdemir, ${ }^{124, \text { aaa }}$ A. Polatoz, ${ }^{124}$ D. Sunar Cerci, ${ }^{124, b b b}$ B. Tali, ${ }^{124, b b b}$ U. G. Tok, ${ }^{124}$ H. Topakli, ${ }^{124, x x}$ S. Turkcapar, ${ }^{124}$ I. S. Zorbakir, ${ }^{124}$ C. Zorbilmez ${ }^{124}$ B. Isildak, ${ }^{125, c c c}$ G. Karapinar, ${ }^{125, \text { ddd }}$ M. Yalvac, ${ }^{125}$ M. Zeyrek, ${ }^{125}$ I. O. Atakisi, ${ }^{126}$ E. Gülmez, ${ }^{126}$ M. Kaya, ${ }^{126, \text { eee }}$ O. Kaya, ${ }^{126, f f f}$ S. Ozkorucuklu, ${ }^{126, g g g}$ S. Tekten, ${ }^{126}$ E. A. Yetkin, ${ }^{126, h h}$ M. N. Agaras, ${ }^{127}$ A. Cakir, ${ }^{127}$ K. Cankocak, ${ }^{127}$ Y. Komurcu, ${ }^{127}$ S. Sen, ${ }^{127, \text { iii }}$ B. Grynyov, ${ }^{128}$ L. Levchuk, ${ }^{129}$ F. Ball, ${ }^{130}$ J. J. Brooke, ${ }^{130}$ D. Burns, ${ }^{130}$ E. Clement, ${ }^{130}$ D. Cussans, ${ }^{130}$ O. Davignon, ${ }^{130}$ H. Flacher, ${ }^{130}$ J. Goldstein, ${ }^{130}$ G. P. Heath, ${ }^{130}$ H. F. Heath, ${ }^{130}$ L. Kreczko, ${ }^{130}$ D. M. Newbold, ${ }^{130, j j j}$ S. Paramesvaran, ${ }^{130}$ B. Penning, ${ }^{130}$ T. Sakuma, ${ }^{130}$ D. Smith, ${ }^{130}$ V. J. Smith,${ }^{130}$ J. Taylor, ${ }^{130}$ A. Titterton, ${ }^{130}$ K. W. Bell, ${ }^{131}$ A. Belyaev, ${ }^{131, \mathrm{kkk}}$ C. Brew, ${ }^{131}$ R. M. Brown, ${ }^{131}$ D. Cieri, ${ }^{131}$ D. J. A. Cockerill, ${ }^{131}$ J. A. Coughlan, ${ }^{131}$ K. Harder, ${ }^{131}$ S. Harper, ${ }^{131}$ J. Linacre, ${ }^{131}$ E. Olaiya, ${ }^{131}$ D. Petyt,${ }^{131}$ C. H. Shepherd-Themistocleous, ${ }^{131}$ A. Thea, ${ }^{131}$ I. R. Tomalin, ${ }^{131}$ T. Williams, ${ }^{131}$ W. J. Womersley, ${ }^{131}$ R. Bainbridge, ${ }^{132}$ P. Bloch, ${ }^{132}$ J. Borg, ${ }^{132}$ S. Breeze, ${ }^{132}$ O. Buchmuller, ${ }^{132}$ A. Bundock, ${ }^{132}$ D. Colling, ${ }^{132}$ P. Dauncey, ${ }^{132}$ G. Davies, ${ }^{132}$ M. Della Negra, ${ }^{132}$ R. Di Maria, ${ }^{132}$ G. Hall ${ }^{132}$ G. Iles, ${ }^{132}$ T. James, ${ }^{132}$ M. Komm, ${ }^{132}$ C. Laner, ${ }^{132}$ L. Lyons, ${ }^{132}$ A.-M. Magnan, ${ }^{132}$ S. Malik, ${ }^{132}$ A. Martelli, ${ }^{132}$ J. Nash, ${ }^{132,111}$ A. Nikitenko, ${ }^{132, \mathrm{~g}}$ V. Palladino ${ }^{132}$ M. Pesaresi ${ }^{132}$ D. M. Raymond, ${ }^{132}$ A. Richards, ${ }^{132}$ A. Rose, ${ }^{132}$ E. Scott, ${ }^{132}$ C. Seez, ${ }^{132}$ A. Shtipliyski, ${ }^{132}$ G. Singh, ${ }^{132}$ M. Stoye, ${ }^{132}$ T. Strebler, ${ }^{132}$ S. Summers, ${ }^{132}$ A. Tapper, ${ }^{132}$ K. Uchida, ${ }^{132}$ T. Virdee, ${ }^{132, p}$ N. Wardle, ${ }^{132}$ D. Winterbottom, ${ }^{132}$ J. Wright, ${ }^{132}$ S. C. Zenz, ${ }^{132}$ J. E. Cole, ${ }^{133}$ P. R. Hobson, ${ }^{133}$ A. Khan, ${ }^{133}$ P. Kyberd, ${ }^{133}$ C. K. Mackay, ${ }^{133}$ A. Morton, ${ }^{133}$ I. D. Reid,${ }^{133}$ L. Teodorescu, ${ }^{133}$ S. Zahid, ${ }^{133}$ K. Call, ${ }^{134}$ J. Dittmann, ${ }^{134}$ K. Hatakeyama, ${ }^{134}$ H. Liu, ${ }^{134}$ C. Madrid ${ }^{134}$ B. McMaster, ${ }^{134}$ N. Pastika, ${ }^{134}$ C. Smith, ${ }^{134}$ R. Bartek, ${ }^{135}$ A. Dominguez, ${ }^{135}$ A. Buccilli, ${ }^{136}$ S. I. Cooper, ${ }^{136}$ C. Henderson, ${ }^{136}$ P. Rumerio, ${ }^{136}$ C. West, ${ }^{136}$ D. Arcaro, ${ }^{137}$ T. Bose, ${ }^{137}$ D. Gastler, ${ }^{137}$ D. Pinna, ${ }^{137}$ D. Rankin, ${ }^{137}$ C. Richardson, ${ }^{137}$ J. Rohlf, ${ }^{137}$ L. Sulak, ${ }^{137}$ D. Zou, ${ }^{137}$ G. Benelli, ${ }^{138}$ X. Coubez, ${ }^{138}$ D. Cutts, ${ }^{138}$ M. Hadley, ${ }^{138}$ J. Hakala, ${ }^{138}$ U. Heintz, ${ }^{138}$ J. M. Hogan, ${ }^{138, m m m}$ K. H. M. Kwok, ${ }^{138}$ E. Laird, ${ }^{138}$ G. Landsberg, ${ }^{138}$ J. Lee,${ }^{138}$ Z. Mao, ${ }^{138}$ M. Narain, ${ }^{138}$ S. Sagir, ${ }^{138, n n n}$ R. Syarif, ${ }^{138}$ E. Usai, ${ }^{138}$ D. Yu, ${ }^{138}$ R. Band,${ }^{139}$ C. Brainerd, ${ }^{139}$ R. Breedon, ${ }^{139}$ D. Burns, ${ }^{139}$ M. Calderon De La Barca Sanchez, ${ }^{139}$ M. Chertok, ${ }^{139}$ J. Conway, ${ }^{139}$ R. Conway, ${ }^{139}$ P. T. Cox, ${ }^{139}$ R. Erbacher, ${ }^{139}$ C. Flores, ${ }^{139}$ G. Funk, ${ }^{139}$ W. Ko, ${ }^{139}$ O. Kukral, ${ }^{139}$ R. Lander ${ }^{139}$ M. Mulhearn, ${ }^{139}$ D. Pellett, ${ }^{139}$ J. Pilot, ${ }^{139}$ S. Shalhout, ${ }^{139}$ M. Shi, ${ }^{139}$ D. Stolp, ${ }^{139}$ D. Taylor, ${ }^{139}$ K. Tos, ${ }^{139}$ M. Tripathi, ${ }^{139}$ Z. Wang, ${ }^{139}$ F. Zhang, ${ }^{139}$ M. Bachtis, ${ }^{140}$ C. Bravo, ${ }^{140}$ R. Cousins, ${ }^{140}$ A. Dasgupta, ${ }^{140}$ A. Florent, ${ }^{140}$ J. Hauser, ${ }^{140}$ M. Ignatenko, ${ }^{140}$ N. Mccoll, ${ }^{140}$ S. Regnard, ${ }^{140}$ D. Saltzberg, ${ }^{140}$ C. Schnaible, ${ }^{140}$ V. Valuev, ${ }^{140}$ E. Bouvier, ${ }^{141}$ K. Burt, ${ }^{141}$ R. Clare,${ }^{141}$ J. W. Gary, ${ }^{141}$ S. M. A. Ghiasi Shirazi, ${ }^{141}$ G. Hanson, ${ }^{141}$ G. Karapostoli, ${ }^{141}$ E. Kennedy, ${ }^{141}$ F. Lacroix, ${ }^{141}$ O. R. Long, ${ }^{141}$ M. Olmedo Negrete, ${ }^{141}$ M. I. Paneva, ${ }^{141}$ W. Si, ${ }^{141}$ 
L. Wang, ${ }^{141}$ H. Wei, ${ }^{141}$ S. Wimpenny, ${ }^{141}$ B. R. Yates, ${ }^{141}$ J. G. Branson, ${ }^{142}$ P. Chang, ${ }^{142}$ S. Cittolin, ${ }^{142}$ M. Derdzinski, ${ }^{142}$ R. Gerosa, ${ }^{142}$ D. Gilbert, ${ }^{142}$ B. Hashemi, ${ }^{142}$ A. Holzner, ${ }^{142}$ D. Klein, ${ }^{142}$ G. Kole, ${ }^{142}$ V. Krutelyov, ${ }^{142}$ J. Letts, ${ }^{142}$ M. Masciovecchio, ${ }^{142}$ D. Olivito, ${ }^{142}$ S. Padhi,${ }^{142}$ M. Pieri, ${ }^{142}$ M. Sani, ${ }^{142}$ V. Sharma, ${ }^{142}$ S. Simon, ${ }^{142}$ M. Tadel,${ }^{142}$ A. Vartak, ${ }^{142}$ S. Wasserbaech, ${ }^{142,000}$ J. Wood, ${ }^{142}$ F. Würthwein, ${ }^{142}$ A. Yagil, ${ }^{142}$ G. Zevi Della Porta, ${ }^{142}$ N. Amin, ${ }^{143}$ R. Bhandari, ${ }^{143}$ C. Campagnari, ${ }^{143}$ M. Citron, ${ }^{143}$ V. Dutta, ${ }^{143}$ M. Franco Sevilla, ${ }^{143}$ L. Gouskos, ${ }^{143}$ R. Heller, ${ }^{143}$ J. Incandela, ${ }^{143}$ A. Ovcharova, ${ }^{143}$ H. Qu ${ }^{143}$ J. Richman, ${ }^{143}$ D. Stuart, ${ }^{143}$ I. Suarez, ${ }^{143}$ S. Wang, ${ }^{143}$ J. Yoo, ${ }^{143}$ D. Anderson, ${ }^{144}$ A. Bornheim, ${ }^{144}$ J. M. Lawhorn, ${ }^{144}$ N. Lu, ${ }^{144}$ H. B. Newman, ${ }^{144}$ T. Q. Nguyen, ${ }^{144}$ M. Spiropulu, ${ }^{144}$ J. R. Vlimant, ${ }^{144}$ R. Wilkinson, ${ }^{144}$ S. Xie, ${ }^{144}$ Z. Zhang, ${ }^{144}$ R. Y. Zhu, ${ }^{144}$ M. B. Andrews, ${ }^{145}$ T. Ferguson, ${ }^{145}$ T. Mudholkar,${ }^{145}$ M. Paulini, ${ }^{145}$ M. Sun, ${ }^{145}$ I. Vorobiev, ${ }^{145}$ M. Weinberg, ${ }^{145}$ J. P. Cumalat, ${ }^{146}$ W. T. Ford, ${ }^{146}$ F. Jensen, ${ }^{146}$ A. Johnson, ${ }^{146}$ E. MacDonald, ${ }^{146}$ T. Mulholland, ${ }^{146}$ R. Patel, ${ }^{146}$ A. Perloff, ${ }^{146}$ K. Stenson, ${ }^{146}$ K. A. Ulmer, ${ }^{146}$ S. R. Wagner, ${ }^{146}$ J. Alexander,${ }^{147}$ J. Chaves, ${ }^{147}$ Y. Cheng, ${ }^{147}$ J. Chu, ${ }^{147}$ A. Datta, ${ }^{147}$ K. Mcdermott, ${ }^{147}$ N. Mirman, ${ }^{147}$ J. R. Patterson, ${ }^{147}$ D. Quach, ${ }^{147}$ A. Rinkevicius, ${ }^{147}$ A. Ryd,${ }^{147}$ L. Skinnari, ${ }^{147}$ L. Soffi, ${ }^{147}$ S. M. Tan, ${ }^{147}$ Z. Tao, ${ }^{147}$ J. Thom, ${ }^{147}$ J. Tucker ${ }^{147}$ P. Wittich, ${ }^{147}$ M. Zientek, ${ }^{147}$ S. Abdullin, ${ }^{148}$ M. Albrow, ${ }^{148}$ M. Alyari, ${ }^{148}$ G. Apollinari, ${ }^{148}$ A. Apresyan, ${ }^{148}$ A. Apyan, ${ }^{148}$ S. Banerjee,${ }^{148}$ L. A. T. Bauerdick, ${ }^{148}$ A. Beretvas, ${ }^{148}$ J. Berryhill, ${ }^{148}$ P. C. Bhat, ${ }^{148}$ K. Burkett, ${ }^{148}$ J. N. Butler, ${ }^{148}$ A. Canepa, ${ }^{148}$ G. B. Cerati, ${ }^{148}$ H. W. K. Cheung, ${ }^{148}$ F. Chlebana, ${ }^{148}$ M. Cremonesi, ${ }^{148}$ J. Duarte, ${ }^{148}$ V. D. Elvira, ${ }^{148}$ J. Freeman, ${ }^{148}$ Z. Gecse, ${ }^{148}$ E. Gottschalk, ${ }^{148}$ L. Gray, ${ }^{148}$ D. Green, ${ }^{148}$ S. Grünendahl, ${ }^{148}$ O. Gutsche, ${ }^{148}$ J. Hanlon, ${ }^{148}$ R. M. Harris, ${ }^{148}$ S. Hasegawa, ${ }^{148}$ J. Hirschauer, ${ }^{148}$ Z. Hu, ${ }^{148}$ B. Jayatilaka, ${ }^{148}$ S. Jindariani, ${ }^{148}$ M. Johnson, ${ }^{148}$ U. Joshi, ${ }^{148}$ B. Klima, ${ }^{148}$ M. J. Kortelainen, ${ }^{148}$ B. Kreis, ${ }^{148}$ S. Lammel, ${ }^{148}$ D. Lincoln, ${ }^{148}$ R. Lipton, ${ }^{148}$ M. Liu, ${ }^{148}$ T. Liu, ${ }^{148}$ J. Lykken, ${ }^{148}$

K. Maeshima, ${ }^{148}$ J. M. Marraffino, ${ }^{148}$ D. Mason, ${ }^{148}$ P. McBride, ${ }^{148}$ P. Merkel,${ }^{148}$ S. Mrenna, ${ }^{148}$ S. Nahn, ${ }^{148}$ V. O’Dell, ${ }^{148}$ K. Pedro, ${ }^{148}$ C. Pena, ${ }^{148}$ O. Prokofyev, ${ }^{148}$ G. Rakness, ${ }^{148}$ L. Ristori, ${ }^{148}$ A. Savoy-Navarro, ${ }^{148, p p p}$ B. Schneider, ${ }^{148}$ E. Sexton-Kennedy, ${ }^{148}$ A. Soha, ${ }^{148}$ W. J. Spalding, ${ }^{148}$ L. Spiegel, ${ }^{148}$ S. Stoynev,${ }^{148}$ J. Strait, ${ }^{148}$ N. Strobbe,${ }^{148}$ L. Taylor, ${ }^{148}$ S. Tkaczyk,${ }^{148}$ N. V. Tran, ${ }^{148}$ L. Uplegger,${ }^{148}$ E. W. Vaandering, ${ }^{148}$ C. Vernieri, ${ }^{148}$ M. Verzocchi, ${ }^{148}$ R. Vidal, ${ }^{148}$ M. Wang, ${ }^{148}$ H. A. Weber, ${ }^{148}$ A. Whitbeck, ${ }^{148}$ D. Acosta, ${ }^{149}$ P. Avery, ${ }^{149}$ P. Bortignon, ${ }^{149}$ D. Bourilkov, ${ }^{149}$ A. Brinkerhoff, ${ }^{149}$ L. Cadamuro, ${ }^{149}$ A. Carnes, ${ }^{149}$ D. Curry, ${ }^{149}$ R. D. Field, ${ }^{149}$ S. V. Gleyzer, ${ }^{149}$ B. M. Joshi, ${ }^{149}$ J. Konigsberg, ${ }^{149}$ A. Korytov, ${ }^{149}$ K. H. Lo, ${ }_{149}$ P. Ma ${ }^{149}$ K. Matchev, ${ }^{149}$ H. Mei, ${ }^{149}$ G. Mitselmakher, ${ }^{149}$ D. Rosenzweig, ${ }^{149}$ K. Shi,${ }^{149}$ D. Sperka, ${ }^{149}$ J. Wang, ${ }^{149}$ S. Wang, ${ }^{149}$ X. Zuo, ${ }^{149}$ Y. R. Joshi,${ }^{150}$ S. Linn, ${ }^{150}$ A. Ackert, ${ }^{151}$ T. Adams, ${ }^{151}$ A. Askew, ${ }^{151}$ S. Hagopian, ${ }^{151}$ V. Hagopian, ${ }^{151}$ K. F. Johnson, ${ }^{151}$ T. Kolberg, ${ }^{151}$ G. Martinez, ${ }^{151}$ T. Perry, ${ }^{151}$ H. Prosper, ${ }^{151}$ A. Saha, ${ }^{151}$ C. Schiber, ${ }^{151}$ R. Yohay, ${ }^{151}$ M. M. Baarmand, ${ }^{152}$ V. Bhopatkar, ${ }^{152}$ S. Colafranceschi, ${ }^{152}$ M. Hohlmann, ${ }^{152}$ D. Noonan, ${ }^{152}$ M. Rahmani, ${ }^{152}$ T. Roy, ${ }^{152}$ F. Yumiceva, ${ }^{152}$ M. R. Adams, ${ }^{153}$ L. Apanasevich, ${ }^{153}$ D. Berry, ${ }^{153}$ R. R. Betts,${ }^{153}$ R. Cavanaugh, ${ }^{153}$ X. Chen, ${ }^{153}$ S. Dittmer, ${ }^{153}$ O. Evdokimov, ${ }^{153}$ C. E. Gerber, ${ }^{153}$ D. A. Hangal, ${ }^{153}$ D. J. Hofman, ${ }^{153}$ K. Jung, ${ }^{153}$ J. Kamin, ${ }^{153}$ C. Mills, ${ }^{153}$

I. D. Sandoval Gonzalez, ${ }^{153}$ M. B. Tonjes, ${ }^{153}$ H. Trauger, ${ }^{153}$ N. Varelas, ${ }^{153}$ H. Wang, ${ }^{153}$ X. Wang, ${ }^{153}$ Z. Wu, ${ }^{153}$ J. Zhang, ${ }^{153}$ M. Alhusseini, ${ }^{154}$ B. Bilki, ${ }^{154, q q q}$ W. Clarida, ${ }^{154}$ K. Dilsiz, ${ }^{154, \text { rrr }}$ S. Durgut, ${ }^{154}$ R. P. Gandrajula, ${ }^{154}$ M. Haytmyradov, ${ }^{154}$ V. Khristenko, ${ }^{154}$ J.-P. Merlo, ${ }^{154}$ A. Mestvirishvili, ${ }^{154}$ A. Moeller, ${ }^{154}$ J. Nachtman, ${ }^{154}$ H. Ogul, ${ }^{154, \text { sss }}$ Y. Onel,,${ }^{154}$ F. Ozok, ${ }^{154, \text { ttt }}$

A. Penzo, ${ }^{154}$ C. Snyder, ${ }^{154}$ E. Tiras, ${ }^{154}$ J. Wetzel,${ }^{154}$ B. Blumenfeld,${ }^{155}$ A. Cocoros, ${ }^{155}$ N. Eminizer, ${ }^{155}$ D. Fehling, ${ }^{155}$ L. Feng, ${ }^{155}$ A. V. Gritsan, ${ }^{155}$ W. T. Hung, ${ }^{155}$ P. Maksimovic, ${ }^{155}$ J. Roskes,${ }^{155}$ U. Sarica,${ }^{155}$ M. Swartz, ${ }^{155}$ M. Xiao, ${ }^{155}$ C. You, ${ }^{155}$ A. Al-bataineh, ${ }^{156}$ P. Baringer, ${ }^{156}$ A. Bean, ${ }^{156}$ S. Boren, ${ }^{156}$ J. Bowen, ${ }^{156}$ A. Bylinkin, ${ }^{156}$ J. Castle, ${ }^{156}$ S. Khalil, ${ }^{156}$ A. Kropivnitskaya, ${ }^{156}$ D. Majumder, ${ }^{156}$ W. Mcbrayer, ${ }^{156}$ M. Murray ${ }^{156}$ C. Rogan, ${ }^{156}$ S. Sanders,${ }^{156}$ E. Schmitz, ${ }^{156}$ J. D. Tapia Takaki, ${ }^{156}$ Q. Wang, ${ }^{156}$ S. Duric, ${ }^{157}$ A. Ivanov,${ }^{157}$ K. Kaadze,${ }^{157}$ D. Kim, ${ }^{157}$ Y. Maravin, ${ }^{157}$ D. R. Mendis, ${ }^{157}$ T. Mitchell, ${ }^{157}$ A. Modak, ${ }^{157}$ A. Mohammadi, ${ }^{157}$ L. K. Saini, ${ }^{157}$ F. Rebassoo, ${ }^{158}$ D. Wright, ${ }^{158}$ A. Baden, ${ }^{159}$ O. Baron, ${ }^{159}$ A. Belloni ${ }^{159}$ S. C. Eno, ${ }^{159}$ Y. Feng, ${ }^{159}$ C. Ferraioli, ${ }^{159}$ N. J. Hadley, ${ }^{159}$ S. Jabeen, ${ }^{159}$ G. Y. Jeng,${ }^{159}$ R. G. Kellogg ${ }^{159}$ J. Kunkle, ${ }^{159}$ A. C. Mignerey, ${ }^{159}$ S. Nabili, ${ }^{159}$ F. Ricci-Tam, ${ }^{159}$ Y. H. Shin, ${ }^{159}$ A. Skuja, ${ }^{159}$ S. C. Tonwar, ${ }^{159}$ K. Wong, ${ }^{159}$ D. Abercrombie, ${ }^{160}$ B. Allen, ${ }^{160}$ V. Azzolini, ${ }^{160}$ A. Baty, ${ }^{160}$ G. Bauer, ${ }^{160}$ R. Bi, ${ }^{160}$ S. Brandt, ${ }^{160}$ W. Busza, ${ }^{160}$ I. A. Cali, ${ }^{160}$ M. D’Alfonso, ${ }^{160}$ Z. Demiragli, ${ }^{160}$ G. Gomez Ceballos, ${ }^{160}$ M. Goncharov,${ }^{160}$ P. Harris, ${ }^{160}$ D. Hsu, ${ }^{160}$ M. Hu ${ }^{160}$ Y. Iiyama, ${ }^{160}$ G. M. Innocenti, ${ }^{160}$ M. Klute, ${ }^{160}$ D. Kovalskyi, ${ }^{160}$ Y.-J. Lee, ${ }^{160}$ P. D. Luckey ${ }^{160}$ B. Maier, ${ }^{160}$ A. C. Marini, ${ }^{160}$ C. Mcginn, ${ }^{160}$ C. Mironov, ${ }^{160}$ S. Narayanan, ${ }^{160}$ X. Niu, ${ }^{160}$ C. Paus, ${ }^{160}$ C. Roland, ${ }^{160}$ G. Roland, ${ }^{160}$ Z. Shi, ${ }^{160}$ G. S. F. Stephans, ${ }^{160}$

K. Sumorok, ${ }^{160}$ K. Tatar, ${ }^{160}$ D. Velicanu, ${ }^{160}$ J. Wang, ${ }^{160}$ T. W. Wang, ${ }^{160}$ B. Wyslouch, ${ }^{160}$ A. C. Benvenuti, ${ }^{161, \dagger}$

R. M. Chatterjee, ${ }^{161}$ A. Evans, ${ }^{161}$ P. Hansen, ${ }^{161}$ J. Hiltbrand, ${ }^{161}$ Sh. Jain, ${ }^{161}$ S. Kalafut, ${ }^{161}$ M. Krohn, ${ }^{161}$ Y. Kubota, ${ }^{161}$ Z. Lesko, ${ }^{161}$ J. Mans, ${ }^{161}$ N. Ruckstuhl, ${ }^{161}$ R. Rusack, ${ }^{161}$ M. A. Wadud, ${ }^{161}$ J. G. Acosta, ${ }^{162}$ S. Oliveros, ${ }^{162}$ E. Avdeeva, ${ }^{163}$ K. Bloom, ${ }^{163}$ D. R. Claes, ${ }^{163}$ C. Fangmeier, ${ }^{163}$ F. Golf, ${ }^{163}$ R. Gonzalez Suarez, ${ }^{163}$ R. Kamalieddin, ${ }^{163}$ I. Kravchenko, ${ }^{163}$ 
J. Monroy, ${ }^{163}$ J. E. Siado, ${ }^{163}$ G. R. Snow, ${ }^{163}$ B. Stieger, ${ }^{163}$ A. Godshalk, ${ }^{164}$ C. Harrington, ${ }^{164}$ I. Iashvili, ${ }^{164}$ A. Kharchilava, ${ }^{164}$ C. Mclean, ${ }^{164}$ D. Nguyen, ${ }^{164}$ A. Parker, ${ }^{164}$ S. Rappoccio, ${ }^{164}$ B. Roozbahani, ${ }^{164}$ G. Alverson, ${ }^{165}$ E. Barberis, ${ }^{165}$ C. Freer, ${ }^{165}$ Y. Haddad ${ }^{165}$ A. Hortiangtham, ${ }^{165}$ D. M. Morse, ${ }^{165}$ T. Orimoto, ${ }^{165}$ R. Teixeira De Lima, ${ }^{165}$ T. Wamorkar, ${ }^{165}$ B. Wang, ${ }^{165}$ A. Wisecarver, ${ }^{165}$ D. Wood, ${ }^{165}$ S. Bhattacharya, ${ }^{166}$ J. Bueghly, ${ }^{166}$ O. Charaf, ${ }^{166}$ K. A. Hahn, ${ }^{166}$ N. Mucia, ${ }^{166}$ N. Odell, ${ }^{166}$ M. H. Schmitt, ${ }^{166}$ K. Sung, ${ }^{166}$ M. Trovato, ${ }^{166}$ M. Velasco, ${ }^{166}$ R. Bucci, ${ }^{167}$ N. Dev, ${ }^{167}$ M. Hildreth, ${ }^{167}$ K. Hurtado Anampa, ${ }^{167}$ C. Jessop, ${ }^{167}$ D. J. Karmgard, ${ }^{167}$ N. Kellams, ${ }^{167}$ K. Lannon, ${ }^{167}$ W. Li, ${ }^{167}$ N. Loukas, ${ }^{167}$ N. Marinelli, ${ }^{167}$ F. Meng, ${ }^{167}$ C. Mueller, ${ }^{167}$ Y. Musienko, ${ }^{167, j \mathrm{j}}$ M. Planer, ${ }^{167}$ A. Reinsvold, ${ }^{167}$ R. Ruchti, ${ }^{167}$ P. Siddireddy, ${ }^{167}$ G. Smith, ${ }^{167}$ S. Taroni, ${ }^{167}$ M. Wayne, ${ }^{167}$ A. Wightman, ${ }^{167}$ M. Wolf,${ }^{167}$ A. Woodard, ${ }^{167}$ J. Alimena, ${ }^{168}$ L. Antonelli, ${ }^{168}$ B. Bylsma, ${ }^{168}$ L. S. Durkin, ${ }^{168}$ S. Flowers, ${ }^{168}$ B. Francis, ${ }^{168}$ C. Hill, ${ }^{168}$ W. Ji, ${ }^{168}$ T. Y. Ling, ${ }^{168}$ W. Luo, ${ }^{168}$ B. L. Winer, ${ }^{168}$ S. Cooperstein,${ }^{169}$ P. Elmer, ${ }^{169}$ J. Hardenbrook, ${ }^{169}$ S. Higginbotham, ${ }^{169}$ A. Kalogeropoulos, ${ }^{169}$ D. Lange, ${ }^{169}$ M. T. Lucchini, ${ }^{169}$ J. Luo, ${ }^{169}$ D. Marlow, ${ }^{169}$ K. Mei, ${ }^{169}$ I. Ojalvo, ${ }^{169}$ J. Olsen, ${ }^{169}$ C. Palmer, ${ }^{169}$ P. Piroué, ${ }^{169}$ J. Salfeld-Nebgen, ${ }^{169}$ D. Stickland, ${ }^{169}$ C. Tully, ${ }^{169}$ Z. Wang, ${ }^{169}$ S. Malik, ${ }^{170}$ S. Norberg, ${ }^{170}$ A. Barker, ${ }^{171}$ V. E. Barnes, ${ }^{171}$ S. Das, ${ }^{171}$ L. Gutay, ${ }^{171}$ M. Jones, ${ }^{171}$ A. W. Jung, ${ }^{171}$ A. Khatiwada, ${ }^{171}$ B. Mahakud, ${ }^{171}$ D. H. Miller, ${ }^{171}$ N. Neumeister, ${ }^{171}$ C. C. Peng, ${ }^{171}$ S. Piperov, ${ }^{171}$ H. Qiu, ${ }^{171}$ J. F. Schulte, ${ }^{171}$ J. Sun, ${ }^{171}$ F. Wang, ${ }^{171}$ R. Xiao, ${ }^{171}$ W. Xie, ${ }^{171}$ T. Cheng, ${ }^{172}$ J. Dolen, ${ }^{172}$ N. Parashar, ${ }^{172}$ Z. Chen, ${ }^{173}$ K. M. Ecklund,${ }^{173}$ S. Freed ${ }^{173}$ F. J. M. Geurts, ${ }^{173}$ M. Kilpatrick, ${ }^{173}$ W. Li,${ }^{173}$ B. P. Padley, ${ }^{173}$ R. Redjimi, ${ }^{173}$ J. Roberts, ${ }^{173}$ J. Rorie, ${ }^{173}$ W. Shi, ${ }^{173}$ Z. Tu, ${ }^{173}$ A. Zhang, ${ }^{173}$ A. Bodek, ${ }^{174}$ P. de Barbaro, ${ }^{174}$ R. Demina, ${ }^{174}$ Y. t. Duh, ${ }^{174}$ J. L. Dulemba, ${ }^{174}$ C. Fallon, ${ }^{174}$ T. Ferbel, ${ }^{174}$ M. Galanti, ${ }^{174}$ A. Garcia-Bellido, ${ }^{174}$ J. Han, ${ }^{174}$ O. Hindrichs, ${ }^{174}$ A. Khukhunaishvili, ${ }^{174}$ E. Ranken, ${ }^{174}$ P. Tan, ${ }^{174}$ R. Taus, ${ }^{174}$ A. Agapitos, ${ }^{175}$ J. P. Chou, ${ }^{175}$ Y. Gershtein, ${ }^{175}$ E. Halkiadakis, ${ }^{175}$ A. Hart, ${ }^{175}$ M. Heindl, ${ }^{175}$ E. Hughes,${ }^{175}$ S. Kaplan, ${ }^{175}$ R. Kunnawalkam Elayavalli, ${ }^{175}$ S. Kyriacou, ${ }^{175}$ A. Lath, ${ }^{175}$ R. Montalvo, ${ }^{175}$ K. Nash,${ }^{175}$ M. Osherson, ${ }^{175}$ H. Saka, ${ }^{175}$ S. Salur, ${ }^{175}$ S. Schnetzer, ${ }^{175}$ D. Sheffield, ${ }^{175}$ S. Somalwar, ${ }^{175}$ R. Stone, ${ }^{175}$ S. Thomas, ${ }^{175}$ P. Thomassen, ${ }^{175}$ M. Walker, ${ }^{175}$ A. G. Delannoy, ${ }^{176}$ J. Heideman, ${ }^{176}$ G. Riley, ${ }^{176}$ S. Spanier, ${ }^{176}$ O. Bouhali, ${ }^{177, \text { uuu }}$ A. Celik, ${ }^{177}$ M. Dalchenko, ${ }^{177}$ M. De Mattia, ${ }^{177}$ A. Delgado, ${ }^{177}$ S. Dildick, ${ }^{177}$ R. Eusebi, ${ }^{177}$ J. Gilmore, ${ }^{177}$ T. Huang, ${ }^{177}$ T. Kamon, ${ }^{177, v v v}$ S. Luo, ${ }^{177}$ R. Mueller, ${ }^{177}$ D. Overton, ${ }^{177}$ L. Perniè, ${ }^{177}$ D. Rathjens, ${ }^{177}$ A. Safonov, ${ }^{177}$ N. Akchurin, ${ }^{178}$ J. Damgov, ${ }^{178}$ F. De Guio, ${ }^{178}$ P. R. Dudero, ${ }^{178}$ S. Kunori, ${ }^{178}$ K. Lamichhane, ${ }^{178}$ S. W. Lee, ${ }^{178}$ T. Mengke, ${ }^{178}$ S. Muthumuni, ${ }^{178}$ T. Peltola,${ }^{178}$ S. Undleeb, ${ }^{178}$ I. Volobouev, ${ }^{178}$ Z. Wang, ${ }^{178}$ S. Greene, ${ }^{179}$ A. Gurrola, ${ }^{179}$ R. Janjam, ${ }^{179}$ W. Johns,${ }^{179}$ C. Maguire,${ }^{179}$ A. Melo, ${ }^{179} \mathrm{H} . \mathrm{Ni},{ }^{179}$ K. Padeken, ${ }^{179}$ J. D. Ruiz Alvarez, ${ }^{179}$ P. Sheldon, ${ }^{179}$ S. Tuo, ${ }^{179}$ J. Velkovska, ${ }^{179}$ M. Verweij, ${ }^{179}$ Q. Xu, ${ }^{179}$ M. W. Arenton, ${ }^{180}$ P. Barria, ${ }^{180}$ B. Cox,${ }^{180}$ R. Hirosky, ${ }^{180}$ M. Joyce, ${ }^{180}$ A. Ledovskoy, ${ }^{180}$ H. Li,${ }^{180}$ C. Neu, ${ }^{180}$ T. Sinthuprasith ${ }^{180}$ Y. Wang, ${ }^{180}$ E. Wolfe, ${ }^{180}$ F. Xia, ${ }^{180}$ R. Harr ${ }^{181}$ P. E. Karchin, ${ }^{181}$ N. Poudyal, ${ }^{181}$ J. Sturdy,${ }^{181}$ P. Thapa ${ }^{181}$ S. Zaleski, ${ }^{181}$ M. Brodski, ${ }^{182}$ J. Buchanan, ${ }^{182}$ C. Caillol, ${ }^{182}$ D. Carlsmith, ${ }^{182}$ S. Dasu, ${ }^{182}$ I. De Bruyn, ${ }^{182}$ L. Dodd ${ }^{182}$ B. Gomber, ${ }^{182}$ M. Grothe, ${ }^{182}$ M. Herndon, ${ }^{182}$ A. Hervé, ${ }^{182}$ U. Hussain, ${ }^{182}$ P. Klabbers, ${ }^{182}$ A. Lanaro, ${ }^{182}$ K. Long, ${ }^{182}$ R. Loveless, ${ }^{182}$ T. Ruggles, ${ }^{182}$ A. Savin, ${ }^{182}$ V. Sharma, ${ }^{182}$ N. Smith, ${ }^{182}$ W. H. Smith, ${ }^{182}$ and N. Woods ${ }^{182}$

\title{
(CMS Collaboration)
}

\author{
${ }^{1}$ Yerevan Physics Institute, Yerevan, Armenia \\ ${ }^{2}$ Institut für Hochenergiephysik, Wien, Austria \\ ${ }^{3}$ Institute for Nuclear Problems, Minsk, Belarus \\ ${ }^{4}$ Universiteit Antwerpen, Antwerpen, Belgium \\ ${ }^{5}$ Vrije Universiteit Brussel, Brussel, Belgium \\ ${ }^{6}$ Université Libre de Bruxelles, Bruxelles, Belgium \\ ${ }^{7}$ Ghent University, Ghent, Belgium \\ ${ }^{8}$ Université Catholique de Louvain, Louvain-la-Neuve, Belgium \\ ${ }^{9}$ Centro Brasileiro de Pesquisas Fisicas, Rio de Janeiro, Brazil \\ ${ }^{10}$ Universidade do Estado do Rio de Janeiro, Rio de Janeiro, Brazil \\ ${ }^{11 a}$ Universidade Estadual Paulista, São Paulo, Brazil \\ ${ }^{11 \mathrm{~b}}$ Universidade Federal do ABC, São Paulo, Brazil \\ ${ }^{12}$ Institute for Nuclear Research and Nuclear Energy, Bulgarian Academy of Sciences, Sofia, Bulgaria \\ ${ }^{13}$ University of Sofia, Sofia, Bulgaria \\ ${ }^{14}$ Beihang University, Beijing, China \\ ${ }^{15}$ Institute of High Energy Physics, Beijing, China \\ ${ }^{16}$ State Key Laboratory of Nuclear Physics and Technology, Peking University, Beijing, China \\ ${ }^{17}$ Tsinghua University, Beijing, China \\ ${ }^{18}$ Universidad de Los Andes, Bogota, Colombia
}


${ }^{19}$ University of Split, Faculty of Electrical Engineering, Mechanical Engineering and Naval Architecture, Split, Croatia

${ }^{20}$ University of Split, Faculty of Science, Split, Croatia

${ }^{21}$ Institute Rudjer Boskovic, Zagreb, Croatia

${ }^{22}$ University of Cyprus, Nicosia, Cyprus

${ }^{23}$ Charles University, Prague, Czech Republic

${ }^{24}$ Escuela Politecnica Nacional, Quito, Ecuador

${ }^{25}$ Universidad San Francisco de Quito, Quito, Ecuador

${ }^{26}$ Academy of Scientific Research and Technology of the Arab Republic of Egypt,

Egyptian Network of High Energy Physics, Cairo, Egypt

${ }^{27}$ National Institute of Chemical Physics and Biophysics, Tallinn, Estonia

${ }^{28}$ Department of Physics, University of Helsinki, Helsinki, Finland

${ }^{29}$ Helsinki Institute of Physics, Helsinki, Finland

${ }^{30}$ Lappeenranta University of Technology, Lappeenranta, Finland

${ }^{31}$ IRFU, CEA, Université Paris-Saclay, Gif-sur-Yvette, France

${ }^{32}$ Laboratoire Leprince-Ringuet, Ecole polytechnique, CNRS/IN2P3, Université Paris-Saclay, Palaiseau, France

${ }^{33}$ Université de Strasbourg, CNRS, IPHC UMR 7178, Strasbourg, France

${ }^{34}$ Centre de Calcul de l'Institut National de Physique Nucleaire et de Physique des Particules, CNRS/IN2P3, Villeurbanne, France

${ }^{35}$ Université de Lyon, Université Claude Bernard Lyon 1, CNRS-IN2P3,

Institut de Physique Nucléaire de Lyon, Villeurbanne, France

${ }^{36}$ Georgian Technical University, Tbilisi, Georgia

${ }^{37}$ Tbilisi State University, Tbilisi, Georgia

${ }^{38}$ RWTH Aachen University, I. Physikalisches Institut, Aachen, Germany

${ }^{39}$ RWTH Aachen University, III. Physikalisches Institut A, Aachen, Germany

${ }^{40}$ RWTH Aachen University, III. Physikalisches Institut B, Aachen, Germany

${ }^{41}$ Deutsches Elektronen-Synchrotron, Hamburg, Germany

${ }^{42}$ University of Hamburg, Hamburg, Germany

${ }^{43}$ Karlsruher Institut fuer Technologie, Karlsruhe, Germany

${ }^{44}$ Institute of Nuclear and Particle Physics (INPP), NCSR Demokritos, Aghia Paraskevi, Greece

${ }^{45}$ National and Kapodistrian University of Athens, Athens, Greece

${ }^{46}$ National Technical University of Athens, Athens, Greece

${ }^{47}$ University of Ioánnina, Ioánnina, Greece

${ }^{48}$ MTA-ELTE Lendület CMS Particle and Nuclear Physics Group, Eötvös Loránd University, Budapest, Hungary

${ }^{49}$ Wigner Research Centre for Physics, Budapest, Hungary

${ }^{50}$ Institute of Nuclear Research ATOMKI, Debrecen, Hungary

${ }^{51}$ Institute of Physics, University of Debrecen, Debrecen, Hungary

${ }^{52}$ Indian Institute of Science (IISc), Bangalore, India

${ }^{53}$ National Institute of Science Education and Research, HBNI, Bhubaneswar, India

${ }^{54}$ Panjab University, Chandigarh, India

${ }^{55}$ University of Delhi, Delhi, India

${ }^{56}$ Saha Institute of Nuclear Physics, HBNI, Kolkata,India

${ }^{57}$ Indian Institute of Technology Madras, Madras, India

${ }^{58}$ Bhabha Atomic Research Centre, Mumbai, India

${ }^{59}$ Tata Institute of Fundamental Research-A, Mumbai, India

${ }^{60}$ Tata Institute of Fundamental Research-B, Mumbai, India

${ }^{61}$ Indian Institute of Science Education and Research (IISER), Pune, India

${ }^{62}$ Institute for Research in Fundamental Sciences (IPM), Tehran, Iran

${ }^{63}$ University College Dublin, Dublin, Ireland

${ }^{64 a}$ INFN Sezione di Bari, Bari, Italy

${ }^{64 \mathrm{~b}}$ Università di Bari, Bari, Italy

${ }^{64 \mathrm{c}}$ Politecnico di Bari, Bari, Italy

${ }^{65 a}$ INFN Sezione di Bologna, Bologna, Italy

${ }^{65 \mathrm{~b}}$ Università di Bologna, Bologna, Italy

${ }^{66 a}$ INFN Sezione di Catania, Catania, Italy

${ }^{66 \mathrm{~b}}$ Università di Catania, Catania, Italy

${ }^{67 \mathrm{a}}$ INFN Sezione di Firenze, Firenze, Italy

${ }^{67 \mathrm{~b}}$ Università di Firenze, Firenze, Italy 
${ }^{68}$ INFN Laboratori Nazionali di Frascati, Frascati, Italy

${ }^{69}$ a INFN Sezione di Genova, Genova, Italy

${ }^{69 \mathrm{~b}}$ Università di Genova, Genova, Italy

${ }^{70 a}$ INFN Sezione di Milano-Bicocca, Milano, Italy

${ }^{70 \mathrm{~b}}$ Università di Milano-Bicocca, Milano, Italy

${ }^{71 \mathrm{a}}$ INFN Sezione di Napoli, Napoli, Italy

${ }^{71 b}$ Università di Napoli 'Federico II', Napoli, Italy

${ }^{71 \mathrm{c}}$ Università della Basilicata, Potenza, Italy

${ }^{71 \mathrm{~d}}$ Università G. Marconi, Roma, Italy

${ }^{72 a}$ INFN Sezione di Padova, Padova, Italy

${ }^{72 \mathrm{~b}}$ Università di Padova, Padova, Italy

${ }^{72 \mathrm{c}}$ Università di Trento, Trento, Italy

${ }^{73 a}$ INFN Sezione di Pavia, Pavia, Italy

${ }^{73 b}$ Università di Pavia, Pavia, Italy

${ }^{74 a}$ INFN Sezione di Perugia, Perugia, Italy

${ }^{74 \mathrm{~b}}$ Università di Perugia, Perugia, Italy

${ }^{75 a}$ INFN Sezione di Pisa, Pisa, Italy

${ }^{75 b}$ Università di Pisa, Pisa, Italy

${ }^{75 c}$ Scuola Normale Superiore di Pisa, Pisa, Italy

${ }^{76 a}$ INFN Sezione di Roma, Rome, Italy

${ }^{76 \mathrm{~b}}$ Sapienza Università di Roma, Rome, Italy

${ }^{77 a}$ INFN Sezione di Torino, Torino, Italy

${ }^{77 \mathrm{~b}}$ Università di Torino, Torino, Italy

${ }^{77 \mathrm{c}}$ Università del Piemonte Orientale, Novara, Italy

${ }^{78 a}$ INFN Sezione di Trieste, Trieste, Italy

${ }^{78 b}$ Università di Trieste, Trieste, Italy

${ }^{79}$ Kyungpook National University, Daegu, Korea

${ }^{80}$ Chonnam National University, Institute for Universe and Elementary Particles, Kwangju, Korea

${ }^{81}$ Hanyang University, Seoul, Korea

${ }^{82}$ Korea University, Seoul, Korea

${ }^{83}$ Sejong University, Seoul, Korea

${ }^{84}$ Seoul National University, Seoul, Korea

${ }^{85}$ University of Seoul, Seoul, Korea

${ }^{86}$ Sungkyunkwan University, Suwon, Korea

${ }^{87}$ Vilnius University, Vilnius, Lithuania

${ }^{88}$ National Centre for Particle Physics, Universiti Malaya, Kuala Lumpur, Malaysia

${ }^{89}$ Universidad de Sonora (UNISON), Hermosillo, Mexico

${ }^{90}$ Centro de Investigacion y de Estudios Avanzados del IPN, Mexico City, Mexico

${ }^{91}$ Universidad Iberoamericana, Mexico City, Mexico

${ }^{92}$ Benemerita Universidad Autonoma de Puebla, Puebla, Mexico

${ }^{93}$ Universidad Autónoma de San Luis Potosí, San Luis Potosí, Mexico

${ }^{94}$ University of Auckland, Auckland, New Zealand

${ }^{95}$ University of Canterbury, Christchurch, New Zealand

${ }^{96}$ National Centre for Physics, Quaid-I-Azam University, Islamabad, Pakistan

${ }^{97}$ National Centre for Nuclear Research, Swierk, Poland

${ }^{98}$ Institute of Experimental Physics, Faculty of Physics, University of Warsaw, Warsaw, Poland

${ }^{99}$ Laboratório de Instrumentação e Física Experimental de Partículas, Lisboa, Portugal

${ }^{100}$ Joint Institute for Nuclear Research, Dubna, Russia

${ }^{101}$ Petersburg Nuclear Physics Institute, Gatchina (St. Petersburg), Russia

${ }^{102}$ Institute for Nuclear Research, Moscow, Russia

${ }^{103}$ Institute for Theoretical and Experimental Physics, Moscow, Russia

${ }^{104}$ Moscow Institute of Physics and Technology, Moscow, Russia

${ }^{105}$ National Research Nuclear University 'Moscow Engineering Physics Institute' (MEPhI),

Moscow, Russia

${ }^{106}$ P.N. Lebedev Physical Institute, Moscow, Russia

${ }^{107}$ Skobeltsyn Institute of Nuclear Physics, Lomonosov Moscow State University, Moscow, Russia

${ }^{108}$ Novosibirsk State University (NSU), Novosibirsk, Russia

${ }^{109}$ Institute for High Energy Physics of National Research Centre 'Kurchatov Institute', Protvino, Russia

${ }^{110}$ National Research Tomsk Polytechnic University, Tomsk, Russia

${ }^{111}$ University of Belgrade, Faculty of Physics and Vinca Institute of Nuclear Sciences, Belgrade, Serbia 
${ }^{112}$ Centro de Investigaciones Energéticas Medioambientales y Tecnológicas (CIEMAT), Madrid, Spain

${ }^{113}$ Universidad Autónoma de Madrid, Madrid, Spain

${ }^{114}$ Universidad de Oviedo, Oviedo, Spain

${ }^{115}$ Instituto de Física de Cantabria (IFCA), CSIC-Universidad de Cantabria, Santander, Spain

${ }^{116}$ University of Ruhuna, Department of Physics, Matara, Sri Lanka

${ }^{117}$ CERN, European Organization for Nuclear Research, Geneva, Switzerland

${ }^{118}$ Paul Scherrer Institut, Villigen, Switzerland

${ }^{119}$ ETH Zurich-Institute for Particle Physics and Astrophysics (IPA), Zurich, Switzerland

${ }^{120}$ Universität Zürich, Zurich, Switzerland

${ }^{121}$ National Central University, Chung-Li, Taiwan

${ }^{122}$ National Taiwan University (NTU), Taipei, Taiwan

${ }^{123}$ Chulalongkorn University, Faculty of Science, Department of Physics, Bangkok, Thailand

${ }^{124}$ Cukurova University, Physics Department, Science and Art Faculty, Adana, Turkey

${ }^{125}$ Middle East Technical University, Physics Department, Ankara, Turkey

${ }^{126}$ Bogazici University, Istanbul, Turkey

${ }^{127}$ Istanbul Technical University, Istanbul, Turkey

${ }^{128}$ Institute for Scintillation Materials of National Academy of Science of Ukraine, Kharkov, Ukraine

${ }^{129}$ National Scientific Center, Kharkov Institute of Physics and Technology, Kharkov, Ukraine

${ }^{130}$ University of Bristol, Bristol, United Kingdom

${ }^{131}$ Rutherford Appleton Laboratory, Didcot, United Kingdom

${ }^{132}$ Imperial College, London, United Kingdom

${ }^{133}$ Brunel University, Uxbridge, United Kingdom

${ }^{134}$ Baylor University, Waco, Texas, USA

${ }^{135}$ Catholic University of America, Washington, DC, USA

${ }^{136}$ The University of Alabama, Tuscaloosa, Alabama, USA

${ }^{137}$ Boston University, Boston, Massachusetts, USA

${ }^{138}$ Brown University, Providence, Rhode Island, USA

${ }^{139}$ University of California, Davis, Davis, California, USA

${ }^{140}$ University of California, Los Angeles, California, USA

${ }^{141}$ University of California, Riverside, Riverside, California, USA

${ }^{142}$ University of California, San Diego, La Jolla, California, USA

${ }^{143}$ University of California, Santa Barbara - Department of Physics, Santa Barbara, California, USA

${ }^{144}$ California Institute of Technology, Pasadena, California, USA

${ }^{145}$ Carnegie Mellon University, Pittsburgh, Pennsylvania, USA

${ }^{146}$ University of Colorado Boulder, Boulder, Colorado, USA

${ }^{147}$ Cornell University, Ithaca, New York, USA

${ }^{148}$ Fermi National Accelerator Laboratory, Batavia, Illinois, USA

${ }^{149}$ University of Florida, Gainesville, Florida, USA

${ }^{150}$ Florida International University, Miami, Florida, USA

${ }^{151}$ Florida State University, Tallahassee, Florida, USA

${ }^{152}$ Florida Institute of Technology, Melbourne, Florida, USA

${ }^{153}$ University of Illinois at Chicago (UIC), Chicago, Illinois, USA

${ }^{154}$ The University of Iowa, Iowa City, Iowa, USA

${ }^{155}$ Johns Hopkins University, Baltimore, Maryland, USA

${ }^{156}$ The University of Kansas, Lawrence, Kansas, USA

${ }^{157}$ Kansas State University, Manhattan, Kansas, USA

${ }^{158}$ Lawrence Livermore National Laboratory, Livermore, California, USA

${ }^{159}$ University of Maryland, College Park, Maryland, USA

${ }^{160}$ Massachusetts Institute of Technology, Cambridge, Massachusetts, USA

${ }^{161}$ University of Minnesota, Minneapolis, Minnesota, USA

${ }^{162}$ University of Mississippi, Oxford, Mississippi, USA

${ }^{163}$ University of Nebraska-Lincoln, Lincoln, Nebraska, USA

${ }^{164}$ State University of New York at Buffalo, Buffalo, New York, USA

${ }^{165}$ Northeastern University, Boston, Massachusetts, USA

${ }^{166}$ Northwestern University, Evanston, Illinois, USA

${ }^{167}$ University of Notre Dame, Notre Dame, Indiana, USA

${ }^{168}$ The Ohio State University, Columbus, Ohio, USA

${ }^{169}$ Princeton University, Princeton, New Jersey, USA

${ }^{170}$ University of Puerto Rico, Mayaguez, Puerto Rico

${ }^{171}$ Purdue University, West Lafayette, Indiana, USA 


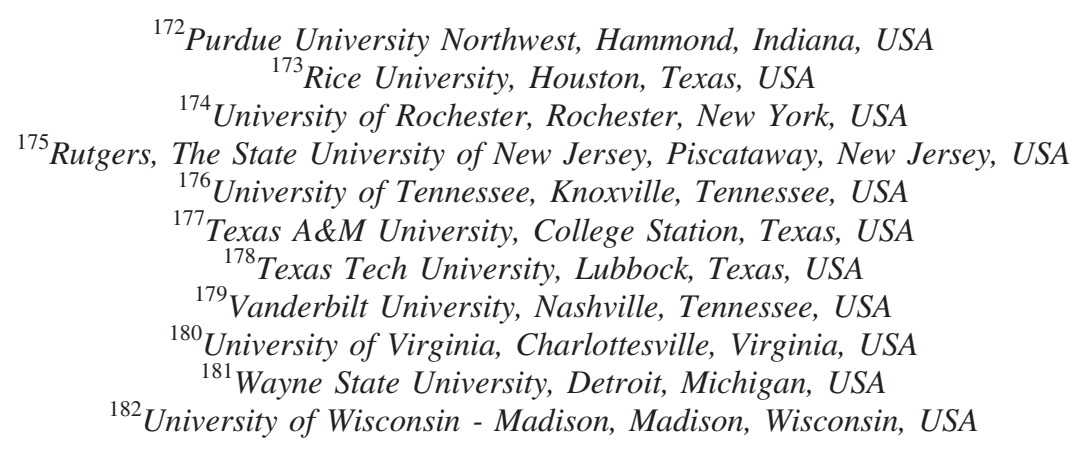

${ }^{\dagger}$ Deceased.

${ }^{a}$ Also at Vienna University of Technology, Vienna, Austria.

${ }^{\mathrm{b}}$ Also at IRFU, CEA, Université Paris-Saclay, Gif-sur-Yvette, France.

${ }^{\mathrm{c}}$ Also at Universidade Estadual de Campinas, Campinas, Brazil.

${ }^{\mathrm{d}}$ Also at Federal University of Rio Grande do Sul, Porto Alegre, Brazil.

${ }^{\mathrm{e}}$ Also at Université Libre de Bruxelles, Bruxelles, Belgium.

${ }^{\mathrm{f}}$ Also at University of Chinese Academy of Sciences, Beijing, China.

${ }^{g}$ Also at Institute for Theoretical and Experimental Physics, Moscow, Russia.

${ }^{\mathrm{h}}$ Also at Joint Institute for Nuclear Research, Dubna, Russia.

${ }^{\mathrm{i}}$ Also at British University in Egypt, Cairo, Egypt.

${ }^{\mathrm{j}}$ Also at Suez University, Suez, Egypt.

${ }^{\mathrm{k}}$ Also at Zewail City of Science and Technology, Zewail, Egypt.

${ }^{1}$ Also at Department of Physics, King Abdulaziz University, Jeddah, Saudi Arabia.

${ }^{\mathrm{m}}$ Also at Université de Haute Alsace, Mulhouse, France.

${ }^{n}$ Also at Skobeltsyn Institute of Nuclear Physics, Lomonosov Moscow State University, Moscow, Russia.

${ }^{\circ}$ Also at Tbilisi State University, Tbilisi, Georgia.

${ }^{\mathrm{p}}$ Also at CERN, European Organization for Nuclear Research, Geneva, Switzerland.

${ }^{\mathrm{q}}$ Also at RWTH Aachen University, III. Physikalisches Institut A, Aachen, Germany.

${ }^{r}$ Also at University of Hamburg, Hamburg, Germany.

${ }^{\mathrm{s}}$ Also at Brandenburg University of Technology, Cottbus, Germany.

${ }^{t}$ Also at Institute of Physics, University of Debrecen, Debrecen, Hungary.

"Also at Institute of Nuclear Research ATOMKI, Debrecen, Hungary.

${ }^{v}$ Also at MTA-ELTE Lendület CMS Particle and Nuclear Physics Group, Eötvös Loránd University, Budapest, Hungary.

${ }^{\mathrm{w}}$ Also at IIT Bhubaneswar, Bhubaneswar, India.

${ }^{\mathrm{x}}$ Also at Institute of Physics, Bhubaneswar, India.

${ }^{\mathrm{y}}$ Also at Shoolini University, Solan, India.

${ }^{z}$ Also at University of Visva-Bharati, Santiniketan, India.

${ }^{\text {aa } A l s o ~ a t ~ I s f a h a n ~ U n i v e r s i t y ~ o f ~ T e c h n o l o g y, ~ I s f a h a n, ~ I r a n . ~}$

${ }^{\mathrm{bb}}$ Also at Plasma Physics Research Center, Science and Research Branch, Islamic Azad University, Tehran, Iran.

${ }^{\mathrm{cc}}$ Also at Università degli Studi di Siena, Siena, Italy.

${ }^{\mathrm{dd}}$ Also at Scuola Normale e Sezione dell'INFN, Pisa, Italy.

${ }^{\text {ee }}$ Also at Kyunghee University, Seoul, Korea.

${ }^{\mathrm{ff}}$ Also at International Islamic University of Malaysia, Kuala Lumpur, Malaysia.

${ }^{\mathrm{gg}}$ Also at Malaysian Nuclear Agency, MOSTI, Kajang, Malaysia.

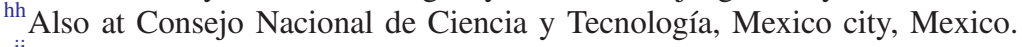

${ }^{i i}$ Also at Warsaw University of Technology, Institute of Electronic Systems, Warsaw, Poland.

${ }^{\mathrm{jj}}$ Also at Institute for Nuclear Research, Moscow, Russia.

${ }^{k k}$ Also at National Research Nuclear University 'Moscow Engineering Physics Institute' (MEPhI), Moscow, Russia.

${ }^{11}$ Also at St. Petersburg State Polytechnical University, St. Petersburg, Russia.

${ }^{\mathrm{mm}}$ Also at University of Florida, Gainesville, Florida, USA.

${ }^{\mathrm{nn}}$ Also at P.N. Lebedev Physical Institute, Moscow, Russia.

${ }^{\circ 0}$ Also at California Institute of Technology, Pasadena, California, USA.

${ }^{\mathrm{pp}}$ Also at Budker Institute of Nuclear Physics, Novosibirsk, Russia.

${ }^{\mathrm{qq}}$ Also at Faculty of Physics, University of Belgrade, Belgrade, Serbia.

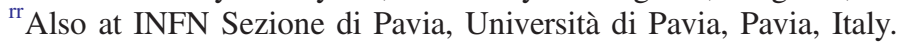

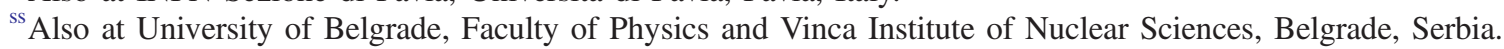

"Also at National and Kapodistrian University of Athens, Athens, Greece.

${ }^{\text {uu }}$ Also at Riga Technical University, Riga, Latvia. 
${ }^{\mathrm{vv}}$ Also at Universität Zürich, Zurich, Switzerland.

${ }^{w w}$ Also at Stefan Meyer Institute for Subatomic Physics, Vienna, Austria.

${ }^{\mathrm{xx}}$ Also at Gaziosmanpasa University, Tokat, Turkey.

${ }^{\text {yy }}$ Also at Istanbul Aydin University, Istanbul, Turkey.

${ }^{\mathrm{zz}}$ Also at Mersin University, Mersin, Turkey.

${ }^{\text {aaa }}$ Also at Piri Reis University, Istanbul, Turkey.

${ }^{\mathrm{bbb}}$ Also at Adiyaman University, Adiyaman, Turkey.

${ }^{\mathrm{ccc}}$ Also at Ozyegin University, Istanbul, Turkey.

${ }^{\text {ddd }}$ Also at Izmir Institute of Technology, Izmir, Turkey.

eee Also at Marmara University, Istanbul, Turkey.

${ }^{\mathrm{fff}}$ Also at Kafkas University, Kars, Turkey.

${ }^{\mathrm{ggg}}$ Also at Istanbul University, Faculty of Science, Istanbul, Turkey.

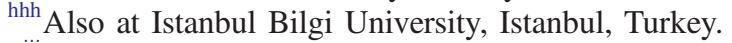

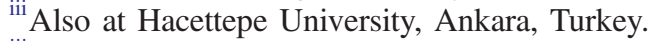

${ }^{\mathrm{jjj}}$ Also at Rutherford Appleton Laboratory, Didcot, United Kingdom.

${ }^{\mathrm{kkk}}$ Also at School of Physics and Astronomy, University of Southampton, Southampton, United Kingdom.

${ }^{111}$ Also at Monash University, Faculty of Science, Clayton, Australia.

${ }^{\mathrm{mmm}}$ Also at Bethel University, St. Paul, Minnesota, USA.

${ }^{\mathrm{nnn}}$ Also at Karamanoğlu Mehmetbey University, Karaman, Turkey.

${ }^{\circ 00}$ Also at Utah Valley University, Orem, Utah, USA.

${ }^{\mathrm{ppp}}$ Also at Purdue University, West Lafayette, Indiana, USA.

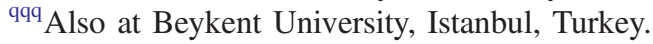

${ }^{\mathrm{rrr}}$ Also at Bingol University, Bingol, Turkey.

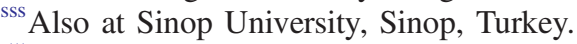

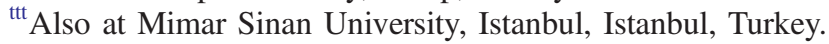

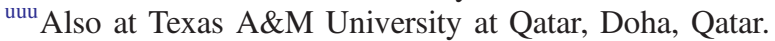

${ }^{\mathrm{vvv}}$ Also at Kyungpook National University, Daegu, Korea. 